\title{
A theoretical development on the entropy of interval-valued intuitionistic fuzzy soft sets based on the distance measure
}

\author{
Yaya Liu ${ }^{1}$, Junfang Luo ${ }^{1}$, Bing Wang ${ }^{2}$, Keyun Qin ${ }^{1 *}$ \\ ${ }^{1}$ College of Mathematics, Southwest Jiaotong University, Cheng Du, 610000, Sichuan, PR China \\ ${ }^{2}$ College of Information Science and Technology, Southwest Jiaotong University, Cheng Du, 610000, Sichuan, \\ PR China \\ E-mail: yayaliu@my.swjtu.edu.cn, junfangluo@163.com,bwang@my.swjtu.edu.cn,keyunqin@263.net
}

Received 16 May 2016

Accepted 19 December 2016

\begin{abstract}
In this work, the axiomatical definition of similarity measure, distance measure and inclusion measure for interval-valued intuitionistic fuzzy soft set (IVIFSSs) are given. An axiomatical definition of entropy measure for IVIFSSs based on distance is firstly proposed, which is consistent with the axiomatical definition of fuzzy entropy of fuzzy sets introduced by De Luca and Termini. By different compositions of aggregation operators and a fuzzy negation operator, we obtain eight general formulae to calculate the distance measures of IVIFSSs based on fuzzy equivalences. Then we discuss the relationships among entropy measures, distance measures, similarity measures and inclusion measures of IVIFSSs. We prove that the presented entropy measures can be transformed into the similarity measures and the inclusion measures of IVIFSSs based on fuzzy equivalences.
\end{abstract}

Keywords: interval-valued intuitionistic fuzzy soft set; entropy; similarity measures; inclusion measures; fuzzy equivalences.

\section{Introduction}

Many new set theories treating imprecision and uncertainty have been proposed since fuzzy sets were introduced by Zadeh ${ }^{1}$. Atanassov's intuitionistic fuzzy sets $^{3}$ (IFSs), vague sets ${ }^{4}$ and intervalvalued fuzzy sets ${ }^{20,21}$ (IVFSs), as extensions of classic fuzzy set theory, are proved to be useful in dealing with imprecision and uncertainty. As a combining concept of IFSs and IVFSs, intervalvalued intuitionistic fuzzy sets (IVIFSs) introduced by Atanassov ${ }^{5}$ greatly furnishes the additional capability to model non-statistical uncertainty by providing a membership interval and a non-membership interval. Therefore, IVIFSs play a significant role in the uncertain system and receives much attention. The concept of soft set theory, which can be used as a general mathematical tool for dealing with uncertainty, is initiated by Molodtsov ${ }^{6}$ in 1999. Since it has been pointed out that classical soft sets are not appropriate to deal with imprecise and fuzzy parameters, some fuzzy (or intuitionistic fuzzy, interval-valued fuzzy) extensions of soft set theory, yielding fuzzy (or intuitionistic fuzzy, interval-valued fuzzy) soft set theory $6,7,8,9,10,11$ has been presented to deal with imprecise and fuzzy parameters. Recently, by combining the intervalvalued intuitionistic fuzzy sets and soft sets, Jiang et al ${ }^{12}$ propose a new soft set model: interval-valued intuitionistic fuzzy soft sets (IVIFSSs). Intuitively,

\footnotetext{
* Corresponding author.
} 
interval-valued intuitionistic fuzzy soft set can be regarded as an interval-valued fuzzy extension of the intuitionistic fuzzy soft set ${ }^{8,9,10}$ or an intuitionistic fuzzy extension of the interval-valued fuzzy soft set 11 .

Some scholars have already noticed and studied entropy measures based on distance for fuzzy sets and extensions of fuzzy sets. $\mathrm{Mi}^{13}$ extended $\mathrm{De} \mathrm{Lu}-$ cas axioms ${ }^{2}$ to introduce an entropy of fuzzy set based on fuzzy distance. Later, Farhadinia ${ }^{16}$ propose a class of entropies of IVFSs based on the distance measure and investigate the relationship between the entropy measure and the similarity measure. Zhang et $\mathrm{al}^{17}$ propose an axiomatical definition of entropy measure for IVIFSs based on distances and discuss the relationship between entropy with similarity and inclusion measure. However, few scholars have paid attention to the entropy measures based on distance for fuzzy (or intuitionistic fuzzy, interval-valued fuzzy, interval-valued intuitionistic fuzzy) extensions of soft sets yet. In this work, we provide an axiomatic definition of entropy based on distance for IVIFSSs and discuss the relationship between entropy measure with similarity, distance and inclusion measures for IVIFSSs. There are several reasons that motivate us to do this research. Firstly, although there are a number of researches regarding entropy measures for hybrid fuzzy set theory, few literatures studied the entropy measure of IVIFSSs; Secondly, the uncertain measures of IVIFSSs have great application potential in many fields such as uncertain system control, decision-making and pattern recognition; Thirdly, the study of relationships between different measure benefits us in achieving as more information as possible through each measure. This new extension not only provides a significant addition to existing theories for handling uncertainties, but also leads to potential areas of further research field and pertinent applications. It is worth noticing that we give a method to construct the distance measures of IVIFSSs by aggregating fuzzy equivalencies and prove that the presented entropy measures can be transformed into the similarity measures and the inclusion measures of IVIF SSs based on fuzzy equivalences.
The structure of this paper is as follows. Section 2 reviews some concepts which are necessary for our paper. Section 3 provides the axiomatic definitions of similarity measure, distance measure and inclusion measure of IVIFSSs, an information entropy based on distance is also introduced to estimate uncertainty in IVIFSSs. Corresponding calculate formulae or construction methods of these measures are also given. In section 4, we investigate the relationship between the entropy measure and other uncertain measures of IVIFSSs, prove that both the similarity measures and the inclusion measures of IVIF SSs can be constructed by entropy measures of IVIF SSs. In section 5, an application of the entropy and the distance measure of IVIFSSs is given. This paper is concluded in Section 6.

\section{Preliminaries}

In this section, we shall recall several definitions which are necessary for our paper.

Let $U$ be the universe of discourse and $P$ be the set of all possible parameters related to the objects in $U$. In the following discussion, we assume that both $U$ and $P$ are nonempty finite sets.

Definition 1. ${ }^{6}$ Let $\mathscr{P}(U)$ be the power set of $U$, a pair $(F, A)$ is called a soft set in the universe $U$, where $A \in P$ and $F$ is a mapping given by

$$
F: A \longrightarrow \mathscr{P}(U)
$$

In other words, the soft set is not a kind of set in ordinary sense, but a parameterized family of subsets of the set $U$. For any parameter $e_{i} \in A, F\left(e_{i}\right) \subseteq$ $U$ may be considered as the set of $e_{i}$ - approximate elements of the soft set $(F, A)$.

Interval-valued intuitionistic fuzzy set was first introduced by Atanassov and Gargov ${ }^{18}$. It is characterized by an interval-valued membership degree and an interval-valued non-membership degree.

Definition 2. ${ }^{5,18}$ An interval-valued intuitionistic fuzzy set on a universe $U$ is an object of the form $A=\left\{\left(x, u_{A}(x), v_{A}(x)\right) / x \in U\right\}$, where $u_{A}$ : $U \longrightarrow \operatorname{Int}([0,1])$ and $v_{A}: U \longrightarrow \operatorname{Int}([0,1])$ satisfy the following condition: $\forall x \in U, \sup \left(u_{A}(x)\right)+$ 
$\sup \left(v_{A}(x)\right) \leqslant 1 .(\operatorname{Int}([0,1])$ stands for the set of all closed subintervals of $[0,1])$.

The class of all interval-valued intuitionistic fuzzy sets (IVIFSs) on $U$ will be denoted by $\operatorname{IVIFS}(U)$.

For an arbitrary set $A \subseteq[0,1]$, define $\bar{A}=\sup A$ and $\underline{A}=\inf A$. The interval-valued intuitionistic fuzzy set $A$ can be written as

$$
\left.A=\left\{\left(x,\left[\underline{u}_{A}(x), \bar{u}_{A}(x)\right)\right],\left[\underline{v}_{A}(x), \bar{v}_{A}(x)\right)\right] / x \in U\right\}
$$

with the condition: $0 \leqslant \bar{u}_{A}(x)+\bar{v}_{A}(x) \leqslant 1$ for all $x \in U$.

The union, intersection and complement of the interval-valued intuitionistic fuzzy sets are defined as follows: let $A, B \in \operatorname{IVIFS}(U)$, then

1) the union of $A$ and $B$ is denoted by $A \cup B$ where

$A \cup B=\left\{\left\langle x,\left[\sup \left(\underline{u}_{A}(x), \underline{u}_{B}(x)\right), \sup \left(\bar{u}_{A}(x), \bar{u}_{B}(x)\right)\right]\right.\right.$, $\left.\left.\left[\inf \left(\underline{v}_{A}(x), \underline{v}_{B}(x)\right), \inf \left(\bar{v}_{A}(x), \bar{v}_{B}(x)\right)\right]\right\rangle \mid x \in U\right\}$.

2)the intersection of $A$ and $B$ is denoted by $A \cap B$ where

$$
\begin{aligned}
& A \cap B=\left\{\left\langlex,\left[\inf \left(\underline{u}_{A}(x), \underline{u}_{B}(x)\right), \inf \left(\bar{u}_{A}(x), \bar{u}_{B}(x)\right],\right.\right.\right. \\
& \left.\left.\left[\sup \left(\underline{v}_{A}(x), \underline{v}_{B}(x)\right), \sup \left(\bar{v}_{A}(x), \bar{v}_{B}(x)\right)\right]\right\rangle \mid x \in U\right\} .
\end{aligned}
$$

3) the complement of $A$ is denoted by $A^{C}$ where

$$
A^{C}=\left\{\left\langle x, v_{A}(x), u_{A}(x)\right\rangle\right\} .
$$

Atanassov $^{5}$ shows that $A \cup B, A \cap B$ and $A^{C}$ are again interval-valued intuitionistic fuzzy sets.

Jiang et al. ${ }^{12}$ define interval-valued intuitionistic fuzzy soft sets (IVIFSSs) by combining intervalvalued intuitionistic fuzzy sets and soft sets, and then give some operations on IVIFSSs.

Definition 3. ${ }^{12}$ A pair $(F, A)$ is an interval-valued intuitionistic fuzzy soft set over $U$, where $A \in P$ and $F$ is a mapping given by

$$
F: A \longrightarrow \operatorname{IVIFS}(U)
$$

The class of all interval-valued intuitionistic fuzzy soft sets over $U$ will be denoted by $\operatorname{IVIFSS}(U)$.
An interval-valued intuitionistic fuzzy soft set is a parameterized family of interval-valued intuitionistic fuzzy subsets of $U$, thus, its universe is the set of all interval-valued intuitionistic fuzzy sets of $U$, i.e., $\operatorname{IVIFS}(U)$. For any parameter $e_{i} \in A, F\left(e_{i}\right)$ is referred as the interval-valued intuitionistic fuzzy value set of parameter $e_{i}$, it can be written as:

$$
\begin{aligned}
& F\left(e_{i}\right)=\left\{\left\langle x_{j}, u_{F\left(e_{i}\right)}\left(x_{j}\right), v_{F\left(e_{i}\right)}\left(x_{j}\right)\right\rangle \mid x_{j} \in U\right\}=\left\{\left\langlex_{j},\right.\right. \\
& \left.\left.\left[\underline{u}_{F\left(e_{i}\right)}\left(x_{j}\right), \bar{u}_{F\left(e_{i}\right)}\left(x_{j}\right)\right],\left[\underline{v}_{F\left(e_{i}\right)}\left(x_{j}\right), \bar{v}_{F\left(e_{i}\right)}\left(x_{j}\right)\right]\right\rangle \mid x_{j} \in U\right\}
\end{aligned}
$$

with the condition $0 \leqslant \bar{u}_{F\left(e_{i}\right)}\left(x_{j}\right)+\bar{v}_{F\left(e_{i}\right)}\left(x_{j}\right) \leqslant 1$. Here, $u_{F\left(e_{i}\right)}\left(x_{j}\right)$ is the interval-valued fuzzy membership degree that object $x_{j}$ holds on parameter $e_{i}, v_{F\left(e_{i}\right)}\left(x_{j}\right)$ is the interval-valued fuzzy nonmembership degree that object $x_{j}$ holds on parameter $e_{i}$.

Definition 4. ${ }^{19}$ Let $\left[a_{1}, b_{1}\right],\left[a_{2}, b_{2}\right] \in \operatorname{Int}([0,1])$, we define

$\left[a_{1}, b_{1}\right] \leqslant\left[a_{2}, b_{2}\right] ;$ iff $a_{1} \leqslant a_{2} ; b_{1} \leqslant b_{2}$; $\left[a_{1}, b_{1}\right] \preceq\left[a_{2}, b_{2}\right] ;$ iff $a_{1} \leqslant a_{2} ; b_{1} \geqslant b_{2}$; $\left[a_{1}, b_{1}\right]=\left[a_{2}, b_{2}\right] ;$ iff $a_{1}=a_{2} ; b_{1}=b_{2}$.

Definition 5. ${ }^{12}$ Let $U$ be an initial universe and $P$ be a set of parameters. Suppose that $A, B \subseteq P$, $(F, A)$ and $(G, B)$ are two interval-valued intuitionistic fuzzy soft sets, we say that $(F, A)$ is an intervalvalued intuitionistic fuzzy soft subset of $(G, B)$ if and only if

(1) $A \subseteq B$;

(2) $\forall e_{i} \in A, \quad F\left(e_{i}\right)$ is an interval-valued intuitionistic fuzzy subset of $G\left(e_{i}\right)$, that is, $\left[\underline{u}_{F\left(e_{i}\right)}\left(x_{j}\right), \bar{u}_{F\left(e_{i}\right)}\left(x_{j}\right)\right] \leqslant\left[\underline{u}_{G\left(e_{i}\right)}\left(x_{j}\right), \bar{u}_{G\left(e_{i}\right)}\left(x_{j}\right)\right]$ and

$\left[\underline{v}_{F\left(e_{i}\right)}\left(x_{j}\right), \bar{v}_{F\left(e_{i}\right)}\left(x_{j}\right)\right] \geqslant\left[\underline{v}_{G\left(e_{i}\right)}\left(x_{j}\right), \bar{v}_{G\left(e_{i}\right)}\left(x_{j}\right)\right]$ for all $x_{j} \in U, e_{i} \in A$.

This relationship is denoted by $(F, A) \subseteq(G, B)$. $(F, A)$ and $(G, B)$ are said to be intuitionistic equal if and only if $(F, A) \supseteq(G, B)$ and $(F, A) \subseteq(G, B)$ at the same time, we write $(F, A)=(G, B)$.

The union and intersection of the interval-valued intuitionistic fuzzy soft sets are defined ${ }^{12}$ as follows: let $(F, A),(G, B) \in \operatorname{IVIFSS}(U)$, then

1) The union of $(F, A)$ and $(G, B)$ is an intervalvalued intuitionistic fuzzy soft set $(H, C)$, where 
$C=A \cup B$ and $e_{i} \in C$.

$u_{H\left(e_{i}\right)}\left(x_{j}\right)=u_{F\left(e_{i}\right)}\left(x_{j}\right), v_{H\left(e_{i}\right)}\left(x_{j}\right)=v_{F\left(e_{i}\right)}\left(x_{j}\right)$, if $e_{i} \in A \backslash B, x_{j} \in U$;

$u_{H\left(e_{i}\right)}\left(x_{j}\right)=u_{G\left(e_{i}\right)}\left(x_{j}\right), \quad v_{H\left(e_{i}\right)}\left(x_{j}\right)=v_{G\left(e_{i}\right)}\left(x_{j}\right)$, if $e_{i} \in B \backslash A, x_{j} \in U$

$u_{H\left(e_{i}\right)}\left(x_{j}\right)=\left[\sup \left(\underline{u}_{F\left(e_{i}\right)}\left(x_{j}\right), \underline{u}_{G\left(e_{i}\right)}\left(x_{j}\right)\right), \sup \left(\bar{u}_{F\left(e_{i}\right)}\left(x_{j}\right)\right.\right.$, $\left.\left.\bar{u}_{G\left(e_{i}\right)}\left(x_{j}\right)\right)\right]$,

$v_{H\left(e_{i}\right)}\left(x_{j}\right)=\left[\inf \left(\underline{v}_{F\left(e_{i}\right)}\left(x_{j}\right), \underline{v}_{G\left(e_{i}\right)}\left(x_{j}\right)\right), \inf \left(\bar{v}_{F\left(e_{i}\right)}\left(x_{j}\right)\right.\right.$, $\left.\left.\bar{v}_{G\left(e_{i}\right)}\left(x_{j}\right)\right)\right]$ if $e_{i} \in A \cap B, x_{j} \in U$.

We denote it by $(F, A) \cup(G, B)=(H, C)$.

2) The intersection of $(F, A)$ and $(G, B)$ is an interval-valued intuitionistic fuzzy soft set $(H, C)$, where $C=A \cup B$ and $e_{i} \in C$.

$u_{H\left(e_{i}\right)}\left(x_{j}\right)=u_{F\left(e_{i}\right)}\left(x_{j}\right), \quad v_{H\left(e_{i}\right)}\left(x_{j}\right)=v_{F\left(e_{i}\right)}\left(x_{j}\right)$, if $e_{i} \in A \backslash B, x_{j} \in U$;

$u_{H\left(e_{i}\right)}\left(x_{j}\right)=u_{G\left(e_{i}\right)}\left(x_{j}\right), v_{H\left(e_{i}\right)}\left(x_{j}\right)=v_{G\left(e_{i}\right)}\left(x_{j}\right)$, if $e_{i} \in B \backslash A, x_{j} \in U$;

$u_{H\left(e_{i}\right)}\left(x_{j}\right)=\left[\inf \left(\underline{u}_{F\left(e_{i}\right)}\left(x_{j}\right), \underline{u}_{G\left(e_{i}\right)}\left(x_{j}\right)\right), \inf \left(\bar{u}_{F\left(e_{i}\right)}\left(x_{j}\right)\right.\right.$, $\left.\left.\bar{u}_{G\left(e_{i}\right)}\left(x_{j}\right)\right)\right]$,

$v_{H\left(e_{i}\right)}\left(x_{j}\right)=\left[\sup \left(\underline{v}_{F\left(e_{i}\right)}\left(x_{j}\right), \underline{v}_{G\left(e_{i}\right)}\left(x_{j}\right)\right), \sup \left(\bar{v}_{F\left(e_{i}\right)}\left(x_{j}\right)\right.\right.$, $\left.\left.\bar{v}_{G\left(e_{i}\right)}\left(x_{j}\right)\right)\right]$,

if $e_{i} \in A \cap B, x_{j} \in U$.

We denote it by $(F, A) \cap(G, B)=(H, C)$.

Definition 6. The relative complement of an interval-valued intuitionistic fuzzy soft set $(F, A)$ is denoted by $(F, A)^{C}$ and is defined by $(F, A)^{C}=$ $\left(F^{C}, A\right)$, where $F^{C}: A \longrightarrow \operatorname{IVIFS}(U)$ is a mapping given by $F^{C}\left(e_{i}\right)=\left\{\left\langle x_{j}, v_{F\left(e_{i}\right)}\left(x_{j}\right), u_{F\left(e_{i}\right)}\left(x_{j}\right)\right\rangle \mid x_{j} \in\right.$ $U\}$ for all $e_{i} \in A$.

Definition 7. ${ }^{12}$ An interval-valued intuitionistic fuzzy soft set $(F, A)$ over $U$ is said to be a null interval-valued intuitionistic fuzzy soft set denoted by $(\emptyset, A)$, if $u_{F\left(e_{i}\right)}\left(x_{j}\right)=[0,0], v_{F\left(e_{i}\right)}\left(x_{j}\right)=[1,1]$ for all $e_{i} \in A, x_{j} \in U$.

Definition 8. ${ }^{12}$ An interval-valued intuitionistic fuzzy soft set $(F, A)$ over $U$ is said to be an absolute interval-valued intu- itionistic fuzzy soft set denoted by $(U, A)$, if $u_{F\left(e_{i}\right)}\left(x_{j}\right)=[1,1], v_{F\left(e_{i}\right)}\left(x_{j}\right)=[0,0]$ for all $e_{i} \in A, x_{j} \in U$.

\section{The distance, similarity, inclusion measure and entropy of IVIFSSS}

\subsection{Axiomatic definitions}

In this subsection, we extend the axiomatic definitions of the distance, similarity, inclusion measure and entropy of IVIF Ss in Ref. ${ }^{17}$ to IVIFSSs.

Definition 9. Let $(F, P),(G, P)$ and $(H, P)$ be interval-valued intuitionistic fuzzy soft sets over $U$, i.e., $(F, P),(G, P),(H, P) \in I V I F S S(U)$. Let $D$ be a mapping $D: \operatorname{IVIFSS}(U) \times \operatorname{IVIFSS}(U) \longrightarrow[0,1]$. If $D((F, P),(G, P))$ satisfies the following properties $((1)-(4))$ :

(1) $D\left((F, P),(F, P)^{C}\right)=1$, if $(F, P)$ is a classical soft set;

(2) $D((F, P),(G, P))=0$, iff $(F, P)=(G, P)$;

(3) $D((F, P),(G, P))=D((G, P),(F, P))$;

(4) $D((F, P),(H, P)) \geqslant D((F, P),(G, P))$ and $D((F, P),(H, P)) \geqslant D((G, P),(H, P)), \quad$ if $(F, P) \subseteq(G, P) \subseteq(H, P)$.

Then $D((F, P),(G, P))$ is a distance measure between interval-valued intuitionistic fuzzy soft sets $(F, P)$ and $(G, P)$.

Definition 10. Let $(F, P),(G, P)$ and $(H, P)$ be interval-valued intuitionistic fuzzy soft sets over $U$, i.e., $(F, P),(G, P),(H, P) \in I V I F S S(U)$. Let $S$ be a mapping $S: \operatorname{IVIFSS}(U) \times \operatorname{IVIFSS}(U) \longrightarrow[0,1]$. If $S((F, P),(G, P))$ satisfies the following properties $((1)-(4))$ :

(1) $S\left((F, P),(F, P)^{C}\right)=0$, if $(F, P)$ is a classical soft set;

(2) $S((F, P),(G, P))=1$, iff $(F, P)=(G, P)$;

(3) $S((F, P),(G, P))=S((G, P),(F, P))$;

(4) $S((F, P),(H, P)) \leqslant S((F, P),(G, P))$ and $S((F, P),(H, P)) \leqslant S((G, P),(H, P)), \quad$ if $(F, P) \subseteq(G, P) \subseteq(H, P)$. 
Then $S((F, P),(G, P))$ is a similarity measure between interval-valued intuitionistic fuzzy soft sets $(F, P)$ and $(G, P)$.

Definition 11. A real function $J: \operatorname{IVIFSS}(U) \times$ $\operatorname{IVIFSS}(U) \longrightarrow[0,1]$ is named as the inclusion measure of interval-valued intuitionistic fuzzy soft sets, if $J$ has the following properties:

(1) If $(F, P)=(U, P),(G, P)=(\emptyset, P)$, then $J((F, P),(G, P))=0$;

(2) $J((F, P),(G, P))=1$, iff $(F, P) \subseteq(G, P)$;

(3) If $(F, P) \subseteq(G, P) \subseteq(H, P)$, then $J((H, P),(F, P)) \leqslant J((G, P),(F, P))$ and $J((H, P),(F, P)) \leqslant J((H, P),(G, P))$.

Then $J((F, P),(G, P))$ is called an inclusion measure of interval-valued intuitionistic fuzzy soft sets.

Definition 12. Let $(Q, P)$ be an interval-valued intuitionistic fuzzy soft set on $U$, s.t. for $\forall e_{i} \in P, Q\left(e_{i}\right)=$ $\left\{\left\langle x_{j},[1 / 2,1 / 2],[1 / 2,1 / 2]\right\rangle \mid x_{j} \in U\right\}$. A real function $I: \operatorname{IVIFSS}(U) \longrightarrow[0,1]$ is called an entropy for interval-valued intuitionistic fuzzy soft sets, if $I$ has the following properties:

(1) $I((F, P))=0$ if $(F, P)$ is a classical soft set;

(2) $I((F, P))=1$ iff $u_{F\left(e_{i}\right)}\left(x_{j}\right)=v_{F\left(e_{i}\right)}\left(x_{j}\right)=$ $[1 / 2,1 / 2], \forall e_{i} \in P, x_{j} \in U$

(3) $I((F, P))=I\left((F, P)^{C}\right)$;

(4) $I((F, P)) \leqslant I((G, P))$, if $D((F, P),(Q, P)) \geqslant$ $D((G, P),(Q, P))$.

Here, the requirement (2) implies that entropy of $(F, P)$ will be maximum if $(F, P)$ is equal to $(Q, P)$; the requirement (4) implies that the closer an interval-valued intuitiionistic fuzzy soft set $(F, P)$ is to $(Q, P)$, the more entropy of $(F, P)$ should decrease.

\subsection{Some general formulae to construct the distance measure of IVIFSSs}

Before giving some general formulae to construct the distance measure of IVIFSSs, we review the notions of aggregation operators and equivalence operators.
Definition 13. ${ }^{14}$ A function $M: \bigcup_{n \in N}[0,1]^{n} \longrightarrow$ $[0,1]$ is an aggregation operator if it satisfies the following properties: for each $n \in N$ and $x_{i}, y_{i} \in[0,1]$,

(1) $M\left(x_{i}\right)=x_{i}$.

(2) $M(\underbrace{0,0, \ldots, 0}_{\mathrm{n} \text { times }})=0$.

(3) $M(\underbrace{1,1, \ldots, 1}_{\mathrm{n} \text { times }})=1$.

(4) $M\left(x_{1}, x_{2}, \ldots x_{n}\right) \leqslant M\left(y_{1}, y_{2}, \ldots y_{n}\right)$ whenever $x_{i} \leqslant y_{i}, \forall i \in\{1,2, \ldots n\}$.

severe

This definition allows us to introduce the following notions:

An aggregation operator $M: \bigcup_{n \in N}[0,1]^{n} \longrightarrow$ $[0,1]$ is called a severe-aggregation operator if it satisfies properties: for each $n \in N$ and $x_{i} \in[0,1](i=$ $\{1,2, \ldots, n\})$,

(5) $M\left(x_{1}, x_{2}, \ldots x_{n}\right)<1$ if $x_{i}<1, \forall i \in\{1,2, \ldots n\}$.

(6) $M\left(x_{1}, x_{2}, \ldots x_{n}\right)>0$ if $x_{i}>0, \forall i \in\{1,2, \ldots n\}$.

An aggregation operator $M: \bigcup_{n \in N}[0,1]^{n} \longrightarrow$ $[0,1]$ is called a top-aggregation operator if it satisfies property: for each $n \in N$ and $x_{i} \in[0,1](i=$ $\{1,2, \ldots, n\})$,

(7) $M\left(x_{1}, x_{2}, \ldots x_{n}\right)=1 \Leftrightarrow x_{i}=1, \forall i \in\{1,2, \ldots n\}$.

An aggregation operator $M: \bigcup_{n \in N}[0,1]^{n} \longrightarrow$ $[0,1]$ is called a bottom-aggregation operator if it satisfies property: for each $n \in N$ and $x_{i} \in[0,1](i=$ $\{1,2, \ldots, n\})$,

(8) $M\left(x_{1}, x_{2}, \ldots x_{n}\right)=0 \Leftrightarrow x_{i}=0, \forall i \in\{1,2, \ldots n\}$.

An aggregation operator $M: \bigcup_{n \in N}[0,1]^{n} \longrightarrow$ $[0,1]$ is called an idempotent-aggregation operator if it satisfies property: for each $n \in N$ and $x \in[0,1]$,

(9) $M(\underbrace{x, x, \ldots, x}_{\mathrm{n} \text { times }})=x$ for $\forall x \in[0,1]$

Example 1. As examples of the severe-aggregation operators, we take: for each $n \in N$ and $x_{i} \in[0,1](i=$ $\{1,2, \ldots, n\})$, 
(1) $M\left(x_{1}, x_{2}, \ldots x_{n}\right)=\frac{1}{n} \sum_{i=1}^{n} x_{i}$.

(2) $M\left(x_{1}, x_{2}, \ldots x_{n}\right)=\lambda \min \left(x_{1}, x_{2}, \ldots, x_{n}\right)+(1-\lambda)$ $\max \left(x_{1}, x_{2}, \ldots, x_{n}\right)$ with $\lambda \in[0,1]$.

(3) $M\left(x_{1}, x_{2}, \ldots x_{n}\right)=\max \left(x_{1}, x_{2}, \ldots, x_{n}\right) /\left(\max \left(x_{1}\right.\right.$, $\left.\left.x_{2}, \ldots, x_{n}\right)+\max \left(1-x_{1}, 1-x_{2}, \ldots, 1-x_{n}\right)\right)$.

As examples of the top-aggregation operators, we take: for each $n \in N$ and $x_{i} \in[0,1](i=\{1,2, \ldots, n\})$,

(1) $M\left(x_{1}, x_{2}, \ldots, x_{n}\right)=\left(\frac{x_{1}^{p}+x_{2}^{p}+\ldots+x_{n}^{p}}{n}\right)^{\frac{1}{p}}, p \geqslant 1$.

(2) $M\left(x_{1}, x_{2}, \ldots, x_{n}\right)=x_{1}^{p} \wedge x_{2}^{p} \wedge \ldots \wedge x_{n}^{p}, p \geqslant 1$.

As examples of the bottom-aggregation operators, we take: for each $n \in N$ and $x_{i} \in[0,1](i=$ $\{1,2, \ldots, n\})$,

(1) $M\left(x_{1}, x_{2}, \ldots, x_{n}\right)=\left(\frac{x_{1}^{p}+x_{2}^{p}+\ldots+x_{n}^{p}}{n}\right)^{\frac{1}{p}}, p \geqslant 1$.

(2) $M\left(x_{1}, x_{2}, \ldots, x_{n}\right)=x_{1}^{p} \vee x_{2}^{p} \vee \ldots \vee x_{n}^{p}, p \geqslant 1$.

As examples of the idempotent-aggregation operators, we take: for each $n \in N$ and $x_{i} \in[0,1](i=$ $\{1,2, \ldots, n\})$,

(1) $M\left(x_{1}, x_{2}, \ldots, x_{n}\right)=\left(\frac{x_{1}^{p}+x_{2}^{p}+\ldots+x_{n}^{p}}{n}\right)^{\frac{1}{p}}, p \geqslant 1$.

(2) $M\left(x_{1}, x_{2}, \ldots x_{n}\right)=\lambda \min \left(x_{1}, x_{2}, \ldots, x_{n}\right)+(1-\lambda)$ $\max \left(x_{1}, x_{2}, \ldots, x_{n}\right)$ with $\lambda \in[0,1]$.

(3) $M\left(x_{1}, x_{2}, \ldots, x_{n}\right)=x_{1} \wedge x_{2} \wedge \ldots \wedge x_{n}$.

(4) $M\left(x_{1}, x_{2}, \ldots, x_{n}\right)=x_{1} \vee x_{2} \vee \ldots \vee x_{n}$

Definition 14. ${ }^{15}$ A function $E:[0,1]^{2} \longrightarrow[0,1]$ is called a fuzzy equivalence if it satisfies the following properties:

(1) $E(x, y)=E(y, x)$ for all $x, y \in[0,1]$.

(2) $E(x, x)=1$ for all $x \in[0,1]$.

(3) $E(0,1)=E(1,0)=0$.

(4) For all $x, y, x^{\prime}, y^{\prime} \in[0,1]$, if $x \leqslant x^{\prime} \leqslant y^{\prime} \leqslant y$, then $E(x, y) \leqslant E\left(x^{\prime}, y^{\prime}\right)$.

In this article, we strength condition (2) to $\left(2^{\prime}\right)$ :

$\left(2^{\prime}\right)$ For all $x, y \in[0,1], E(x, y)=1$ iff $x=y$.
Definition 15. ${ }^{22}$ If a decreasing function $n$ : $[0,1] \longrightarrow[0,1]$ satisfies the boundary conditions $n(0)=1$ and $n(1)=0$, then $n$ is called a fuzzy negation.

If a fuzzy negation $n:[0,1] \longrightarrow[0,1]$ is a strictly decreasing function, it is called a strict fuzzy negation in this work.

By the compositions of three severe-aggregation operators and a strict fuzzy negation operator, we obtain eight general formulae to calculate the distance measures of IVIFSSs based on fuzzy equivalencies.

Definition 16. Given $U=\left\{x_{1}, x_{2}, \ldots, x_{n}\right\}$ and $P=$ $\left\{e_{1}, e_{2}, \ldots, e_{m}\right\}$. Let $M_{k}(k=1,2,3)$ be severeaggregation operators. Let $E_{l}(l=1,2,3,4)$ be fuzzy equivalence operators and $f$ be a strict fuzzy negation. Suppose $D_{q}(q=1,2, \ldots, 8): \operatorname{IVIFSS}(U) \times$ $\operatorname{IVIFSS}(U) \longrightarrow[0,1]$ are functions defined for all $(F, P),(G, P) \in I V I F S S(U)$ as follows: for any $e_{i} \in$ $P, x_{j} \in U$,

$D_{1}((F, P),(G, P))=\stackrel{n}{M_{1}} \underset{j=1}{\stackrel{m}{M}} \stackrel{M}{2}=1_{3}\left(M_{3}\left(f\left(E_{1}\left(\bar{u}_{F\left(e_{i}\right)}\left(x_{j}\right)\right.\right.\right.\right.$,

$\left.\bar{u}_{G\left(e_{i}\right)}\left(x_{j}\right)\right), f\left(E_{2}\left(\underline{u}_{F\left(e_{i}\right)}\left(x_{j}\right), \underline{u}_{G\left(e_{i}\right)}\left(x_{j}\right)\right), f\left(E_{3}\left(\bar{v}_{F\left(e_{i}\right)}\left(x_{j}\right)\right.\right.\right.$,

$\left.\left.\left.\left.\bar{v}_{G\left(e_{i}\right)}\left(x_{j}\right)\right), f\left(E_{4}\left(\underline{v}_{F\left(e_{i}\right)}\left(x_{j}\right), \underline{v}_{G\left(e_{i}\right)}\left(x_{j}\right)\right)\right)\right)\right)\right)$.

$D_{2}((F, P),(G, P))=\stackrel{m}{M} \stackrel{m}{2}_{i=1} \stackrel{n}{M}\left(M_{1}\left(M_{3}\left(f\left(E_{1}\left(\bar{u}_{F\left(e_{i}\right)}\left(x_{j}\right)\right.\right.\right.\right.\right.$,

$\left.\bar{u}_{G\left(e_{i}\right)}\left(x_{j}\right)\right), f\left(E_{2}\left(\underline{u}_{F\left(e_{i}\right)}\left(x_{j}\right), \underline{u}_{G\left(e_{i}\right)}\left(x_{j}\right)\right), f\left(E_{3}\left(\bar{v}_{F\left(e_{i}\right)}\left(x_{j}\right)\right.\right.\right.$,

$\left.\left.\left.\left.\bar{v}_{G\left(e_{i}\right)}\left(x_{j}\right)\right), f\left(E_{4}\left(\underline{v}_{F\left(e_{i}\right)}\left(x_{j}\right), \underline{v}_{G\left(e_{i}\right)}\left(x_{j}\right)\right)\right)\right)\right)\right)$.

$D_{3}((F, P),(G, P))=\stackrel{n}{M_{1}} \stackrel{m}{M}_{i=1}^{m}\left(f\left(M_{3}\left(E_{1}\left(\bar{u}_{F\left(e_{i}\right)}\left(x_{j}\right)\right.\right.\right.\right.$,

$\left.\bar{u}_{G\left(e_{i}\right)}\left(x_{j}\right)\right), E_{2}\left(\underline{u}_{F\left(e_{i}\right)}\left(x_{j}\right), \underline{u}_{G\left(e_{i}\right)}\left(x_{j}\right)\right), E_{3}\left(\bar{v}_{F\left(e_{i}\right)}\left(x_{j}\right)\right.$,

$\left.\left.\left.\left.\left.\bar{v}_{G\left(e_{i}\right)}\left(x_{j}\right)\right), E_{4}\left(\underline{v}_{F\left(e_{i}\right)}\left(x_{j}\right), \underline{v}_{G\left(e_{i}\right)}\left(x_{j}\right)\right)\right)\right)\right)\right)$.

$D_{4}((F, P),(G, P))=\stackrel{m}{M_{2}} \underset{i=1}{\stackrel{n}{M}}(f=1$

$\left.\bar{u}_{G\left(e_{i}\right)}\left(x_{j}\right)\right), E_{2}\left(\underline{u}_{F\left(e_{i}\right)}\left(x_{j}\right), \underline{u}_{G\left(e_{i}\right)}\left(x_{j}\right)\right), E_{3}\left(\bar{v}_{F\left(e_{i}\right)}\left(x_{j}\right)\right.$,

$\left.\left.\left.\left.\left.\bar{v}_{G\left(e_{i}\right)}\left(x_{j}\right)\right), E_{4}\left(\underline{v}_{F\left(e_{i}\right)}\left(x_{j}\right), \underline{v}_{G\left(e_{i}\right)}\left(x_{j}\right)\right)\right)\right)\right)\right)$. 


$$
\begin{aligned}
& D_{5}((F, P),(G, P))=\stackrel{n}{M}_{j=1}^{n}\left(\underset{i=1}{\stackrel{m}{M_{2}}\left(M _ { 3 } \left(E _ { 1 } \left(\bar{u}_{F\left(e_{i}\right)}\left(x_{j}\right),\right.\right.\right.}\right. \\
& \left.\bar{u}_{G\left(e_{i}\right)}\left(x_{j}\right)\right), E_{2}\left(\underline{u}_{F\left(e_{i}\right)}\left(x_{j}\right), \underline{u}_{G\left(e_{i}\right)}\left(x_{j}\right)\right), E_{3}\left(\bar{v}_{F\left(e_{i}\right)}\left(x_{j}\right),\right. \\
& \left.\left.\left.\left.\left.\bar{v}_{G\left(e_{i}\right)}\left(x_{j}\right)\right), E_{4}\left(\underline{v}_{F\left(e_{i}\right)}\left(x_{j}\right), \underline{v}_{G\left(e_{i}\right)}\left(x_{j}\right)\right)\right)\right)\right)\right) .
\end{aligned}
$$

$$
\begin{aligned}
& D_{6}((F, P),(G, P))=\stackrel{m}{M}_{i=1}^{m}\left(f \underset{j=1}{\stackrel{n}{M}\left(M _ { 3 } \left(E _ { 1 } \left(\bar{u}_{F\left(e_{i}\right)}\left(x_{j}\right),\right.\right.\right.}\right. \\
& \left.\bar{u}_{G\left(e_{i}\right)}\left(x_{j}\right)\right), E_{2}\left(\underline{u}_{F\left(e_{i}\right)}\left(x_{j}\right), \underline{u}_{G\left(e_{i}\right)}\left(x_{j}\right)\right), E_{3}\left(\bar{v}_{F\left(e_{i}\right)}\left(x_{j}\right),\right. \\
& \left.\left.\left.\left.\left.\bar{v}_{G\left(e_{i}\right)}\left(x_{j}\right)\right), E_{4}\left(\underline{v}_{F\left(e_{i}\right)}\left(x_{j}\right), \underline{v}_{G\left(e_{i}\right)}\left(x_{j}\right)\right)\right)\right)\right)\right) .
\end{aligned}
$$

$$
\begin{aligned}
& D_{7}((F, P),(G, P))=\underset{j=1}{\stackrel{n}{M_{1}}\left(M _ { i = 1 } ^ { m } \left(M _ { 3 } \left(E _ { 1 } \left(\bar{u}_{F\left(e_{i}\right)}\left(x_{j}\right),\right.\right.\right.\right.} \\
& \left.\bar{u}_{G\left(e_{i}\right)}\left(x_{j}\right)\right), E_{2}\left(\underline{u}_{F\left(e_{i}\right)}\left(x_{j}\right), \underline{u}_{G\left(e_{i}\right)}\left(x_{j}\right)\right), E_{3}\left(\bar{v}_{F\left(e_{i}\right)}\left(x_{j}\right),\right. \\
& \left.\left.\left.\left.\left.\bar{v}_{G\left(e_{i}\right)}\left(x_{j}\right)\right), E_{4}\left(\underline{v}_{F\left(e_{i}\right)}\left(x_{j}\right), \underline{v}_{G\left(e_{i}\right)}\left(x_{j}\right)\right)\right)\right)\right)\right) .
\end{aligned}
$$

$$
\begin{aligned}
& D_{8}((F, P),(G, P))=\underset{i=1}{\stackrel{m}{M_{2}}\left(\stackrel { n } { M } _ { j = 1 } ^ { n } \left(M _ { 3 } \left(E _ { 1 } \left(\bar{u}_{F\left(e_{i}\right)}\left(x_{j}\right),\right.\right.\right.\right.} \\
& \left.\bar{u}_{G\left(e_{i}\right)}\left(x_{j}\right)\right), E_{2}\left(\underline{u}_{F\left(e_{i}\right)}\left(x_{j}\right), \underline{u}_{G\left(e_{i}\right)}\left(x_{j}\right)\right), E_{3}\left(\bar{v}_{F\left(e_{i}\right)}\left(x_{j}\right),\right. \\
& \left.\left.\left.\left.\bar{v}_{G\left(e_{i}\right)}\left(x_{j}\right)\right), E_{4}\left(\underline{v}_{F\left(e_{i}\right)}\left(x_{j}\right), \underline{\underline{v}}_{G\left(e_{i}\right)}\left(x_{j}\right)\right)\right)\right)\right) .
\end{aligned}
$$

Theorem 1. $D_{q}((F, P),(G, P))(q \in\{1,2, \ldots 8\})$ in Definition 16 are distance measures between interval-valued intuitionistic fuzzy soft sets $(F, P)$ and $(G, P)$.

Proof. (1) If $(F, P)$ is a classical soft set, we have

$\left[\underline{u}_{F\left(e_{i}\right)}\left(x_{j}\right), \bar{u}_{F\left(e_{i}\right)}\left(x_{j}\right)\right]=[1,1],\left[\underline{v}_{F\left(e_{i}\right)}\left(x_{j}\right), \bar{v}_{F\left(e_{i}\right)}\left(x_{j}\right)\right]=$ $[0,0]$ or

$\left[\underline{u}_{F\left(e_{i}\right)}\left(x_{j}\right), \bar{u}_{F\left(e_{i}\right)}\left(x_{j}\right)\right]=[0,0],\left[\underline{v}_{F\left(e_{i}\right)}\left(x_{j}\right), \bar{v}_{F\left(e_{i}\right)}\left(x_{j}\right)\right]=$ $[1,1], \forall e_{i} \in P, x_{j} \in U$.

Then we get

$\left[\underline{u}_{F^{C}\left(e_{i}\right)}\left(x_{j}\right), \bar{u}_{F^{C}\left(e_{i}\right)}\left(x_{j}\right)\right]=[0,0],\left[\underline{v}_{F^{C}\left(e_{i}\right)}\left(x_{j}\right), \bar{v}_{F^{C}\left(e_{i}\right)}\left(x_{j}\right)\right]$ $=[1,1]$ or

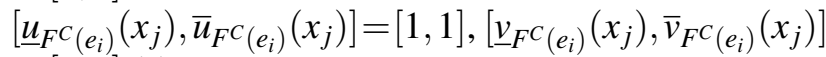
$=[0,0], \forall e_{i} \in P, x_{j} \in U$.

By property (3) of fuzzy equivalence operators, we have

$E_{1}\left(\bar{u}_{F\left(e_{i}\right)}\left(x_{j}\right), \bar{u}_{F^{C}\left(e_{i}\right)}\left(x_{j}\right)\right)=E_{2}\left(\underline{u}_{F\left(e_{i}\right)}\left(x_{j}\right), \underline{u}_{F^{C}\left(e_{i}\right)}\left(x_{j}\right)\right)$

$=E_{3}\left(\bar{v}_{F\left(e_{i}\right)}\left(x_{j}\right), \bar{v}_{F^{C}\left(e_{i}\right)}\left(x_{j}\right)\right)=E_{4}\left(\underline{v}_{F\left(e_{i}\right)}\left(x_{j}\right), \underline{v}_{F^{C}\left(e_{i}\right)}\left(x_{j}\right)\right)$ $=0, \forall e_{i} \in P, x_{j} \in U$.

Thus, we have
$D_{q}((F, P),(G, P))=1(q \in\{1,2, \ldots 8\})$.

(2) If $(F, P)=(G, P)$, it is obviously that $D_{q}((F, P),(G, P))=0(q \in\{1,2, \ldots 8\})$.

For $q \in\{1,2, \ldots 8\}$, assume that $D_{q}((F, P),(G, P))=$ 0 , if there exists a $e_{i} \in P$, and a $x_{j} \in U$, s.t.

$E_{1}\left(\bar{u}_{F\left(e_{i}\right)}\left(x_{j}\right), \bar{u}_{G\left(e_{i}\right)}\left(x_{j}\right)\right)<1$ or

$E_{2}\left(\underline{u}_{F\left(e_{i}\right)}\left(x_{j}\right), \underline{u}_{G\left(e_{i}\right)}\left(x_{j}\right)\right)<1$ or

$E_{3}\left(\bar{v}_{F\left(e_{i}\right)}\left(x_{j}\right), \bar{v}_{G\left(e_{i}\right)}\left(x_{j}\right)\right)<1$ or

$\left.E_{4}\left(\underline{v}_{F\left(e_{i}\right)}\left(x_{j}\right), \underline{v}_{G\left(e_{i}\right)}\left(x_{j}\right)\right)\right)<1$,

since $f$ is a strict fuzzy negation, we get $D_{q}((F, P),(G, P))>0$. It is a contradiction.

So, we have

$E_{1}\left(\bar{u}_{F\left(e_{i}\right)}\left(x_{j}\right), \bar{u}_{G\left(e_{i}\right)}\left(x_{j}\right)\right)=E_{2}\left(\underline{u}_{F\left(e_{i}\right)}\left(x_{j}\right), \underline{u}_{G\left(e_{i}\right)}\left(x_{j}\right)\right)=$ $\left.E_{3}\left(\bar{v}_{F\left(e_{i}\right)}\left(x_{j}\right), \bar{v}_{G\left(e_{i}\right)}\left(x_{j}\right)\right)=E_{4}\left(\underline{v}_{F\left(e_{i}\right)}\left(x_{j}\right), \underline{v}_{G\left(e_{i}\right)}\left(x_{j}\right)\right)\right)=$ $1, \forall e_{i} \in P, x_{j} \in U$.

Thus, we have for any $e_{i} \in P, x_{j} \in U$,

$\bar{u}_{F\left(e_{i}\right)}\left(x_{j}\right)=\bar{u}_{G\left(e_{i}\right)}\left(x_{j}\right), \underline{u}_{F\left(e_{i}\right)}\left(x_{j}\right)=\underline{u}_{G\left(e_{i}\right)}\left(x_{j}\right)$,

$\bar{v}_{F\left(e_{i}\right)}\left(x_{j}\right)=\bar{v}_{G\left(e_{i}\right)}\left(x_{j}\right), \underline{v}_{F\left(e_{i}\right)}\left(x_{j}\right)=\underline{v}_{G\left(e_{i}\right)}\left(x_{j}\right)$,

that is, $(F, P)=(G, P)$.

(3) By the commutative law of the fuzzy equivalence operators, we can easily get that

$D_{q}((F, P),(G, P))=D_{q}((G, P),(F, P))(q \quad \in$ $\{1,2, \ldots 8\})$.

(4) Since $(F, P) \subseteq(G, P) \subseteq(H, P)$, we have for any $e_{i} \in P, x_{j} \in U$,

$\left[\underline{u}_{F\left(e_{i}\right)}\left(x_{j}\right), \bar{u}_{F\left(e_{i}\right)}\left(x_{j}\right)\right] \leqslant\left[\underline{u}_{G\left(e_{i}\right)}\left(x_{j}\right), \bar{u}_{G\left(e_{i}\right)}\left(x_{j}\right)\right] \leqslant$ $\left[\underline{u}_{H\left(e_{i}\right)}\left(x_{j}\right), \bar{u}_{H\left(e_{i}\right)}\left(x_{j}\right)\right]$,

$\left[\underline{v}_{F\left(e_{i}\right)}\left(x_{j}\right), \bar{v}_{F\left(e_{i}\right)}\left(x_{j}\right)\right] \geqslant\left[\underline{v}_{G\left(e_{i}\right)}\left(x_{j}\right), \bar{v}_{G\left(e_{i}\right)}\left(x_{j}\right)\right] \geqslant$ $\left[\underline{v}_{H\left(e_{i}\right)}\left(x_{j}\right), \bar{v}_{H\left(e_{i}\right)}\left(x_{j}\right)\right]$.

By the property of fuzzy equivalence operators, we get for any $e_{i} \in P, x_{j} \in U$,

$E_{1}\left(\bar{u}_{F\left(e_{i}\right)}\left(x_{j}\right), \bar{u}_{H\left(e_{i}\right)}\left(x_{j}\right)\right) \leqslant E_{1}\left(\bar{u}_{F\left(e_{i}\right)}\left(x_{j}\right), \bar{u}_{G\left(e_{i}\right)}\left(x_{j}\right)\right)$, $E_{2}\left(\underline{u}_{F\left(e_{i}\right)}\left(x_{j}\right), \underline{u}_{H\left(e_{i}\right)}\left(x_{j}\right)\right) \leqslant E_{2}\left(\underline{u}_{F\left(e_{i}\right)}\left(x_{j}\right), \underline{u}_{G\left(e_{i}\right)}\left(x_{j}\right)\right)$, $E_{3}\left(\bar{v}_{F\left(e_{i}\right)}\left(x_{j}\right), \bar{v}_{H\left(e_{i}\right)}\left(x_{j}\right)\right) \leqslant E_{3}\left(\bar{v}_{F\left(e_{i}\right)}\left(x_{j}\right), \bar{v}_{G\left(e_{i}\right)}\left(x_{j}\right)\right)$, $E_{4}\left(\underline{v}_{F\left(e_{i}\right)}\left(x_{j}\right), \underline{v}_{H\left(e_{i}\right)}\left(x_{j}\right)\right) \leqslant E_{4}\left(\underline{v}_{F\left(e_{i}\right)}\left(x_{j}\right), \underline{v}_{G\left(e_{i}\right)}\left(x_{j}\right)\right)$. Thus, $D_{q}((F, P),(H, P)) \geqslant D_{q}((F, P),(G, P))(q \in$ $\{1,2, \ldots 8\})$.

Remark 1. All of the distance measures for IVIFSSs are discussed on discrete universes here, the cases for continuous universes can be researched similarly.

Remark 2. If the IVIFSSS degenerate to IVIFSs, the distance measures of IVIFSSs degenerate to the corresponding distance measures of IVIFSs.

Example 2. Considering $(F, P),(G, P) \in$ 


\section{$\operatorname{IVIFSS}(U)$, let}

(1) $M_{1}\left(x_{1}, x_{2}, \ldots x_{n}\right)=M_{2}\left(x_{1}, x_{2}, \ldots x_{n}\right)=\frac{1}{n} \sum_{i=1}^{n} x_{i}$, $x_{i} \in[0,1], \forall n \in N$;

(2) $E_{1}\left(x_{1}, x_{2}\right)=E_{2}\left(x_{1}, x_{2}\right)=E_{3}\left(x_{1}, x_{2}\right)=$ $E_{4}\left(x_{1}, x_{2}\right)=1-\left|x_{1}-x_{2}\right|$, for any $x_{1}, x_{2} \in$ $[0,1]$;

(3) $f(x)=1-x, \forall x \in[0,1]$,

then, we may construct the following distance measures for IVIF SSs by Eq.(2) in Definition 16.

(1) Let $M_{3}\left(x_{1}, x_{2}, x_{3}, x_{4}\right)=\left[\frac{1}{4}\left(x_{1}^{2}+x_{2}^{2}+x_{3}^{2}+x_{4}^{2}\right)\right]^{\frac{1}{2}}$, then we get the Normalized Euclidean distance

$$
\begin{aligned}
& d_{1}((F, P),(G, P))=\left\{\frac { 1 } { 4 m n } \sum _ { i = 1 } ^ { m } \sum _ { j = 1 } ^ { n } \left[\left(\bar{u}_{F\left(e_{i}\right)}\left(x_{j}\right)-\bar{u}_{G\left(e_{i}\right)}\left(x_{j}\right)\right)^{2}\right.\right. \\
& +\left(\underline{u}_{F\left(e_{i}\right)}\left(x_{j}\right)-\underline{u}_{G\left(e_{i}\right)}\left(x_{j}\right)\right)^{2}+\left(\bar{v}_{F\left(e_{i}\right)}\left(x_{j}\right)-\bar{v}_{G\left(e_{i}\right)}\left(x_{j}\right)\right)^{2} \\
& \left.\left.+\left(\underline{v}_{F\left(e_{i}\right)}\left(x_{j}\right)-\underline{v}_{G\left(e_{i}\right)}\left(x_{j}\right)\right)^{2}\right]\right\}^{\frac{1}{2}} .
\end{aligned}
$$

(2) Let $M_{3}\left(x_{1}, x_{2}, x_{3}, x_{4}\right)=\frac{1}{4}\left(x_{1}+x_{2}+x_{3}+x_{4}\right)$, then we get the Normalized hamming distance

$$
\begin{aligned}
& d_{2}((F, P),(G, P))=\frac{1}{4 m n} \sum_{j=1}^{n} \sum_{i=1}^{m}\left[\left|\bar{u}_{F\left(e_{i}\right)}\left(x_{j}\right)-\bar{u}_{G\left(e_{i}\right)}\left(x_{j}\right)\right|\right. \\
& +\left|\underline{u}_{F\left(e_{i}\right)}\left(x_{j}\right)-\underline{u}_{G\left(e_{i}\right)}\left(x_{j}\right)\right|+\left|\bar{v}_{F\left(e_{i}\right)}\left(x_{j}\right)-\bar{v}_{G\left(e_{i}\right)}\left(x_{j}\right)\right| \\
& \left.\left.+\mid \underline{v}_{F\left(e_{i}\right)}\left(x_{j}\right)-\underline{v}_{G\left(e_{i}\right)}\left(x_{j}\right)\right) \mid\right] .
\end{aligned}
$$

(3) Let $M_{3}\left(x_{1}, x_{2}, x_{3}, x_{4}\right)=\frac{1}{2}\left(x_{1} \vee x_{2}+x_{3} \vee x_{4}\right)$, then we get the Normalized hamming distance measure induced by Hausdorff metric

$d_{3}((F, P),(G, P))=\frac{1}{2 m n} \sum_{j=1}^{n} \sum_{i=1}^{m}\left[\left(\left|\bar{u}_{F\left(e_{i}\right)}\left(x_{j}\right)-\bar{u}_{G\left(e_{i}\right)}\left(x_{j}\right)\right|\right.\right.$

$\left.\vee\left|\underline{u}_{F\left(e_{i}\right)}\left(x_{j}\right)-\underline{u}_{G\left(e_{i}\right)}\left(x_{j}\right)\right|\right)+\left(\left|\bar{v}_{F\left(e_{i}\right)}\left(x_{j}\right)-\bar{v}_{G\left(e_{i}\right)}\left(x_{j}\right)\right|\right.$ $\left.\left.\left.\vee \mid \underline{v}_{F\left(e_{i}\right)}\left(x_{j}\right)-\underline{v}_{G\left(e_{i}\right)}\left(x_{j}\right)\right) \mid\right)\right]$.

(4) Let $M_{3}\left(x_{1}, x_{2}, x_{3}, x_{4}\right)=x_{1}^{2} \vee x_{2}^{2} \vee x_{3}^{2} \vee x_{4}^{2}$, then we get the fourth distance

$$
\begin{aligned}
& d_{4}((F, P),(G, P))=\frac{1}{m n} \sum_{j=1}^{n} \sum_{i=1}^{m}\left[\left(\bar{u}_{F\left(e_{i}\right)}\left(x_{j}\right)-\bar{u}_{G\left(e_{i}\right)}\left(x_{j}\right)\right)^{2}\right. \\
& \vee\left(\underline{u}_{F\left(e_{i}\right)}\left(x_{j}\right)-\underline{u}_{G\left(e_{i}\right)}\left(x_{j}\right)\right)^{2} \vee\left(\bar{v}_{F\left(e_{i}\right)}\left(x_{j}\right)-\bar{v}_{G\left(e_{i}\right)}\left(x_{j}\right)\right)^{2} \\
& \left.\vee\left({ }_{-} v_{F\left(e_{i}\right)}\left(x_{j}\right)-\underline{v}_{G\left(e_{i}\right)}\left(x_{j}\right)\right)^{2}\right] .
\end{aligned}
$$

(5) Let $M_{3}\left(x_{1}, x_{2}, x_{3}, x_{4}\right)=\left[\frac{1}{4}\left(x_{1}^{3}+x_{2}^{3}+x_{3}^{3}+x_{4}^{3}\right)\right]^{\frac{1}{3}}$, then we get the fifth distance

$$
\begin{aligned}
& d_{5}((F, P),(G, P))=\left\{\frac { 1 } { 4 m n } \sum _ { i = 1 } ^ { m } \sum _ { j = 1 } ^ { n } \left[\left(\bar{u}_{F\left(e_{i}\right)}\left(x_{j}\right)-\bar{u}_{G\left(e_{i}\right)}\left(x_{j}\right)\right)^{3}\right.\right. \\
& +\left(\underline{u}_{F\left(e_{i}\right)}\left(x_{j}\right)-\underline{u}_{G\left(e_{i}\right)}\left(x_{j}\right)\right)^{3}+\left(\bar{v}_{F\left(e_{i}\right)}\left(x_{j}\right)-\bar{v}_{G\left(e_{i}\right)}\left(x_{j}\right)\right)^{3} \\
& \left.\left.+\left(\underline{v}_{F\left(e_{i}\right)}\left(x_{j}\right)-\underline{v}_{G\left(e_{i}\right)}\left(x_{j}\right)\right)^{3}\right]\right\}^{\frac{1}{3}} .
\end{aligned}
$$

If $(F, P),(G, P) \in \operatorname{IVIFSS}(U)$ are reduced to $F, G \in I V I F S(U)$, we get the following distance measures of IVIFSs. Note that the similarity measures $d_{1}^{\prime}-d_{3}^{\prime}$ of IVIFSs have been proposed in Ref. ${ }^{17}$, whereas $d_{4}^{\prime}-d_{5}^{\prime}$ are new for IVIF Ss.

(1) The Normalized Euclidean distance

$$
\begin{aligned}
& d_{1}^{\prime}(F, G)=\left\{\frac { 1 } { 4 n } \sum _ { j = 1 } ^ { n } \left[\left(\bar{u}_{F}\left(x_{j}\right)-\bar{u}_{G}\left(x_{j}\right)\right)^{2}+\left(\underline{u}_{F}\left(x_{j}\right)-\right.\right.\right. \\
& \left.\left.\left.\underline{u}_{G}\left(x_{j}\right)\right)^{2}+\left(\bar{v}_{F}\left(x_{j}\right)-\bar{v}_{G}\left(x_{j}\right)\right)^{2}+\left(\underline{v}_{F}\left(x_{j}\right)-\underline{v}_{G}\left(x_{j}\right)\right)^{2}\right]\right\}^{\frac{1}{2}} .
\end{aligned}
$$

(2) The Normalized hamming distance

$$
\begin{aligned}
& d_{2}^{\prime}(F, G)=\frac{1}{4 n} \sum_{j=1}^{n}\left[\left|\bar{u}_{F}\left(x_{j}\right)-\bar{u}_{G}\left(x_{j}\right)\right|+\left|\underline{u}_{F}\left(x_{j}\right)-\underline{u}_{G}\left(x_{j}\right)\right|\right. \\
& \left.\left.+\left|\bar{v}_{F}\left(x_{j}\right)-\bar{v}_{G}\left(x_{j}\right)\right|+\mid \underline{v}_{F}\left(x_{j}\right)-\underline{v}_{G}\left(x_{j}\right)\right) \mid\right] .
\end{aligned}
$$

(3) The Normalized hamming distance measure induced by Hausdorff metric

$$
\begin{aligned}
& d_{3}^{\prime}(F, G)=\frac{1}{2 n} \sum_{j=1}^{n}\left[\left(\left|\bar{u}_{F}\left(x_{j}\right)-\bar{u}_{G}\left(x_{j}\right)\right| \vee\left|\underline{u}_{F}\left(x_{j}\right)-\underline{u}_{G}\left(x_{j}\right)\right|\right)\right. \\
& \left.\left.+\left(\left|\bar{v}_{F}\left(x_{j}\right)-\bar{v}_{G}\left(x_{j}\right)\right| \vee \mid \underline{v}_{F}\left(x_{j}\right)-\underline{v}_{G}\left(x_{j}\right)\right) \mid\right)\right] .
\end{aligned}
$$

(4) Let $M_{3}\left(x_{1}, x_{2}, x_{3}, x_{4}\right)=x_{1}^{2} \vee x_{2}^{2} \vee x_{3}^{2} \vee x_{4}^{2}$, then we get the fourth distance

$$
\begin{aligned}
& d_{4}^{\prime}(F, G)=\frac{1}{n} \sum_{j=1}^{n}\left[\left(\bar{u}_{F}\left(x_{j}\right)-\bar{u}_{G}\left(x_{j}\right)\right)^{2} \vee\left(\underline{u}_{F}\left(x_{j}\right)-\underline{u}_{G}\left(x_{j}\right)\right)^{2}\right. \\
& \left.\vee\left(\bar{v}_{F}\left(x_{j}\right)-\bar{v}_{G}\left(x_{j}\right)\right)^{2} \vee\left(\underline{v}_{F}\left(x_{j}\right)-\underline{v}_{G}\left(x_{j}\right)\right)^{2}\right] .
\end{aligned}
$$

(5) Let $M_{3}\left(x_{1}, x_{2}, x_{3}, x_{4}\right)=\left[\frac{1}{4}\left(x_{1}^{3}+x_{2}^{3}+x_{3}^{3}+x_{4}^{3}\right)\right]^{\frac{1}{3}}$, then we get the fifth distance

$$
\begin{aligned}
& \left.d_{5}^{\prime}(F, G)\right)=\left\{\frac { 1 } { 4 n } \sum _ { j = 1 } ^ { n } \left[\left(\bar{u}_{F}\left(x_{j}\right)-\bar{u}_{G}\left(x_{j}\right)^{3}+\left(\underline{u}_{F}\left(x_{j}\right)-\right.\right.\right.\right. \\
& \left.\left.\underline{u}_{G}\left(x_{j}\right)^{3}+\left(\bar{v}_{F}\left(x_{j}\right)-\bar{v}_{G}\left(x_{j}\right)\right)^{3}+\left(\underline{v}_{F}\left(x_{j}\right)-\underline{v}_{G}\left(x_{j}\right)\right)^{3}\right]\right\}^{\frac{1}{3}} .
\end{aligned}
$$


Example 3. Considering $(F, P),(G, P) \quad \in$ $\operatorname{IVIFSS}(U)$, let

(1) $M_{1}\left(x_{1}, x_{2}, \ldots, x_{n}\right)=\lambda \min \left(x_{1}, x_{2}, \ldots, x_{n}\right)+(1-$ $\lambda) \max \left(x_{1}, x_{2}, \ldots, x_{n}\right)$ with $\lambda \in[0,1]$,

$M_{2}\left(x_{1}, x_{2}, \ldots, x_{n}\right)=\frac{1}{n} \sum_{i=1}^{n} x_{i}$,

$M_{3}\left(x_{1}, x_{2}, \ldots, x_{n}\right)=x_{1} \vee x_{2}, \ldots, \vee x_{n}$,

for each $n \in N$ and $x_{i} \in[0,1], i \in\{1,2, \ldots, n\}$.

(2) $E_{1}\left(x_{1}, x_{2}\right)=E_{2}\left(x_{1}, x_{2}\right)=1-\left|x_{1}^{2}-x_{2}^{2}\right|$, $E_{3}\left(x_{1}, x_{2}\right)=E_{4}\left(x_{1}, x_{2}\right)=\frac{2 x_{1} x_{2}}{x_{1}^{2}+x_{2}^{2}}$ for any $x_{1}, x_{2} \in[0,1]$.

(3) $f(x)=1-x$, for any $x \in[0,1]$.

We may construct the distance measure for IVIF SSS by Eq.(3) in Definition 16 as follows.

$d_{6}((F, P),(G, P))=\lambda \min \left(\alpha_{1}, \alpha_{2}, \ldots, \alpha_{n}\right)+(1-$ $\lambda) \max \left(\alpha_{1}, \alpha_{2}, \ldots, \alpha_{n}\right)$,

where $\lambda \in[0,1]$ and $\alpha_{j}=\frac{1}{m} \sum_{i=1}^{m}\{1-[(1-$ $\left.\left|\bar{u}_{F\left(e_{i}\right)}\left(x_{j}\right)^{2}-\bar{u}_{G\left(e_{i}\right)}\left(x_{j}\right)^{2}\right|\right) \vee\left(1-\mid \underline{u}_{F\left(e_{i}\right)}\left(x_{j}\right)^{2}-\right.$ $\left.\left.\left.\underline{u}_{G\left(e_{i}\right)}\left(x_{j}\right)^{2} \mid\right) \vee \frac{2 \bar{v}_{F\left(e_{i}\right)}\left(x_{j}\right) \bar{v}_{G\left(e_{i}\right)}\left(x_{j}\right)}{\overline{\bar{v}}_{F\left(e_{i}\right)}\left(x_{j}\right)^{2}+\bar{v}_{G\left(e_{i}\right)}\left(x_{j}\right)^{2}} \vee \frac{\left.2 \underline{v}_{F\left(e_{i}\right)}\right)\left(x_{j}\right) \underline{v}_{G\left(e_{i}\right)}\left(x_{j}\right)}{\underline{\underline{v}}_{F\left(e_{i}\right)}\left(x_{j}\right)^{2}+\underline{v}_{G\left(e_{i}\right)}\left(x_{j}\right)^{2}}\right]\right\}$, $(j=1,2, \ldots, n)$.

\section{Relationships between distance, similarity, inclusion measures and entropy for IVIF SSs}

\subsection{Transformation of distance measures into similarity measures for IVIF SSS}

Theorem 2. Let $f^{\prime}$ be a strict fuzzy negation and $D$ be a distance measure of interval-valued intuitionistic fuzzy soft sets. Then a similarity measure $S$ of interval-valued intuitionistic fuzzy soft sets can be deduced from the distance measure $D$ as follows:

$$
S((F, P)(G, P))=f^{\prime}(D((F, P),(G, P)))
$$

Remark 3. If we take the strict fuzzy negation $f^{\prime}(x)=1-x$ for all $x \in[0,1]$, by the distance measures $D_{i}((F, P),(G, P))(1 \leqslant i \leqslant 8)$ given in Definition 16 , we can generate the corresponding similarity measures of interval-valued intuitionistic fuzzy soft sets as $S_{i}((F, P),(G, P))=1-$ $D_{i}((F, P),(G, P)),(1 \leqslant i \leqslant 8)$.

Example 4. Considering the distance measure given in Example 3, take $f^{\prime}(x)=1-x$, one can get a similarity measure of IVIFSSs as follows.
$S((F, P),(G, P))=1-\left[\lambda \min \left(\alpha_{1}, \alpha_{2}, \ldots, \alpha_{n}\right)+(1-\right.$ $\left.\lambda) \max \left(\alpha_{1}, \alpha_{2}, \ldots, \alpha_{n}\right)\right]$,

where $\lambda \in[0,1]$ and $\alpha_{j}=\frac{1}{m} \sum_{i=1}^{m}\{1-[(1-$ $\left.\left|\bar{u}_{F\left(e_{i}\right)}\left(x_{j}\right)^{2}-\bar{u}_{G\left(e_{i}\right)}\left(x_{j}\right)^{2}\right|\right) \vee\left(1-\mid \underline{u}_{F\left(e_{i}\right)}\left(x_{j}\right)^{2}-\right.$ $\left.\left.\left.\underline{u}_{G\left(e_{i}\right)}\left(x_{j}\right)^{2} \mid\right) \vee \frac{2 \bar{v}_{F\left(e_{i}\right)}\left(x_{j}\right) \bar{v}_{G\left(e_{i}\right)}\left(x_{j}\right)}{\bar{v}_{F\left(e_{i}\right)}\left(x_{j}\right)^{2}+\bar{v}_{G\left(e_{i}\right)}\left(x_{j}\right)^{2}} \vee \frac{2 \underline{v}_{F\left(e_{i}\right)}\left(x_{j}\right) \underline{v}_{G\left(e_{i}\right.}\left(x_{j}\right)}{\left.\underline{v}_{F\left(e_{i}\right)}\left(x_{j}\right)^{2}+\underline{v}_{G\left(e_{i}\right)}\right)\left(x_{j}\right)^{2}}\right]\right\}$, $(j=1,2, \ldots, n)$.

\subsection{Transformation of distance measures into entropies for IVIF SSs}

Now we present a transformation method for constructing entropy of IVIF SSs based on the distance measure of IVIFSSs as follows.

Theorem 3. Let $(Q, P)$ be an interval-valued intuitionistic fuzzy soft set on $U$, s.t. for any $e_{i} \in P$, $\left.Q\left(e_{i}\right)=\left\{\left\langle x_{j},[1 / 2,1 / 2]\right),[1 / 2,1 / 2]\right\rangle \mid x_{j} \in U\right\}$. Suppose that

(1) for each $p \in\{1,2,3\}, M_{p}$ is both a bottomaggregation operator and an idempotentaggregation operator;

(2) $M_{3}\left(x_{1}, x_{2}, x_{3}, x_{4}\right)=M_{3}\left(x_{3}, x_{4}, x_{1}, x_{2}\right)$ for $x_{1}, x_{2}, x_{3}, x_{4} \in[0,1]$;

(3) $E_{1}\left(x_{1}, x_{2}\right)=E_{2}\left(x_{1}, x_{2}\right)=E_{3}\left(x_{1}, x_{2}\right)=$ $E_{4}\left(x_{1}, x_{2}\right)=1-\left|x_{1}-x_{2}\right|$ for any $x_{1}, x_{2} \in[0,1]$;

(4) $f(x)=1-x$, for any $x \in[0,1]$;

(5) $D_{1}((F, P),(Q, P))$ and $D_{2}((F, P),(Q, P))$ are distance measures between $(F, P)$ and $(Q, P)$ constructed by Eq.(1) and Eq.(2) in Definition 16 , respectively;

(6) $f^{\prime}$ is a strict fuzzy negation,

then for any $(F, P) \in \operatorname{IVIFSS}(U)$,

$$
I_{q}((F, P))=f^{\prime}\left(2 D_{q}((F, P),(Q, P))\right)(q=1,2)
$$

are entropies for interval-valued intuitionistic fuzzy soft sets.

Proof. It is sufficient to show that $I((F, P))$ satisfies the requirements (1)-(4) listed in Definition 12. (1) If $(F, P)$ is a classical soft set, we have $\left[\underline{u}_{F\left(e_{i}\right)}\left(x_{j}\right), \bar{u}_{F\left(e_{i}\right)}\left(x_{j}\right)\right]=[1,1],\left[\underline{v}_{F\left(e_{i}\right)}\left(x_{j}\right), \bar{v}_{F\left(e_{i}\right)}\left(x_{j}\right)\right]=$ $[0,0]$ or $\left[\underline{u}_{F\left(e_{i}\right)}\left(x_{j}\right), \bar{u}_{F\left(e_{i}\right)}\left(x_{j}\right)\right]=[0,0],\left[\underline{v}_{F\left(e_{i}\right)}\left(x_{j}\right), \bar{v}_{F\left(e_{i}\right)}\left(x_{j}\right)\right]=$ 
$[1,1], \forall e_{i} \in P, x_{j} \in U$.

Since $E_{1}\left(x_{1}, x_{2}\right)=E_{2}\left(x_{1}, x_{2}\right)=E_{3}\left(x_{1}, x_{2}\right)=$ $E_{4}\left(x_{1}, x_{2}\right)=1-\left|x_{1}-x_{2}\right|$ for any $x_{1}, x_{2} \in[0,1]$, and $f(x)=1-x$ for any $x \in[0,1]$,

we have $f\left(E_{1}\left(\bar{u}_{F\left(e_{i}\right)}\left(x_{j}\right), \frac{1}{2}\right)\right)=f\left(E_{2}\left(\underline{u}_{F\left(e_{i}\right)}\left(x_{j}\right), \frac{1}{2}\right)\right)=$ $f\left(E_{3}\left(\bar{v}_{F\left(e_{i}\right)}\left(x_{j}\right), \frac{1}{2}\right)\right)=f\left(E_{4}\left(\underline{v}_{F\left(e_{i}\right)}\left(x_{j}\right), \frac{1}{2}\right)\right)=\frac{1}{2}$,

$\forall e_{i} \in P, x_{j} \in U$.

Since $M_{p}(p=1,2,3)$ is an idempotent-aggregation operator, we have $D_{q}((F, P),(Q, P))=\frac{1}{2}(q=1,2)$, i.e., $2 D_{q}((F, P),(Q, P))=1(q=1,2)$.

Thus, we get $I_{q}((F, P))=f^{\prime}(1)=0(q=1,2)$.

(2) Since $M_{p}(p=1,2,3)$ is a bottom-aggregation operator and $f^{\prime}$ is a strict fuzzy negation, we get

$I_{q}((F, P))=1(q=1,2)$

$\Leftrightarrow 2 D_{q}((F, P),(Q, P))=0(q=1,2)$

$\Leftrightarrow D_{q}((F, P),(Q, P))=0(q=1,2)$

$\Leftrightarrow f\left(E_{1}\left(\bar{u}_{F\left(e_{i}\right)}\left(x_{j}\right), \frac{1}{2}\right)\right)=f\left(E_{2}\left(\underline{u}_{F\left(e_{i}\right)}\left(x_{j}\right), \frac{1}{2}\right)\right)=$ $f\left(E_{3}\left(\bar{v}_{F\left(e_{i}\right)}\left(x_{j}\right), \frac{1}{2}\right)\right)=f\left(E_{4}\left(\underline{v}_{F\left(e_{i}\right)}\left(x_{j}\right), \frac{1}{2}\right)\right)=0$, $\forall e_{i} \in P, x_{j} \in U$.

$\Leftrightarrow E_{1}\left(\bar{u}_{F\left(e_{i}\right)}\left(x_{j}\right), \frac{1}{2}\right)=E_{2}\left(\underline{u}_{F\left(e_{i}\right)}\left(x_{j}\right), \frac{1}{2}\right)=$

$E_{3}\left(\bar{v}_{F\left(e_{i}\right)}\left(x_{j}\right), \frac{1}{2}\right)=E_{4}\left(\underline{v}_{F\left(e_{i}\right)}\left(x_{j}\right), \frac{1}{2}\right)=1, \forall e_{i} \in P$, $x_{j} \in U$.

$\Leftrightarrow u_{F\left(e_{i}\right)}\left(x_{j}\right)=v_{F\left(e_{i}\right)}\left(x_{j}\right)=\left[\frac{1}{2}, \frac{1}{2}\right], \forall e_{i} \in P, x_{j} \in U$.

(3) For any $e_{i} \in P$,

if $F\left(e_{i}\right)=\left\langle x_{j}, u_{F\left(e_{i}\right)}\left(x_{j}\right), v_{F\left(e_{i}\right)}\left(x_{j}\right)\right\rangle, \forall x_{j} \in U$,

then $F^{C}\left(e_{i}\right)=\left\langle x_{j}, v_{F\left(e_{i}\right)}\left(x_{j}\right), u_{F\left(e_{i}\right)}\left(x_{j}\right)\right\rangle, \forall x_{j} \in U$.

Since $E_{1}\left(x_{1}, x_{2}\right)=E_{2}\left(x_{1}, x_{2}\right)=E_{3}\left(x_{1}, x_{2}\right)=$

$E_{4}\left(x_{1}, x_{2}\right)$ for any $x_{1}, x_{2} \in[0,1]$, we have

$E_{1}\left(\bar{u}_{F\left(e_{i}\right)}\left(x_{j}\right), \frac{1}{2}\right)=E_{3}\left(\bar{u}_{F\left(e_{i}\right)}\left(x_{j}\right), \frac{1}{2}\right)$,

$E_{2}\left(\underline{u}_{F\left(e_{i}\right)}\left(x_{j}\right), \frac{1}{2}\right)=E_{4}\left(\underline{u}_{F\left(e_{i}\right)}\left(x_{j}\right), \frac{1}{2}\right)$,

$E_{3}\left(\bar{v}_{F\left(e_{i}\right)}\left(x_{j}\right), \frac{1}{2}\right)=E_{1}\left(\bar{v}_{F\left(e_{i}\right)}\left(x_{j}\right), \frac{1}{2}\right)$,

$E_{4}\left(\underline{v}_{F\left(e_{i}\right)}\left(x_{j}\right), \frac{1}{2}\right)=E_{2}\left(\underline{v}_{F\left(e_{i}\right)}\left(x_{j}\right), \frac{1}{2}\right)$,

$\forall e_{i} \in P, x_{j} \in U$.

Since $M_{3}\left(x_{1}, x_{2}, x_{3}, x_{4}\right)=M_{3}\left(x_{3}, x_{4}, x_{1}, x_{2}\right)$ for any $x_{1}, x_{2}, x_{3}, x_{4} \in[0,1]$, we have

$M_{3}\left(f\left(E_{1}\left(\bar{u}_{F\left(e_{i}\right)}\left(x_{j}\right), \frac{1}{2}\right)\right), f\left(E_{2}\left(\underline{u}_{F\left(e_{i}\right)}\left(x_{j}\right), \frac{1}{2}\right)\right)\right.$,

$\left.f\left(E_{3}\left(\bar{v}_{F\left(e_{i}\right)}\left(x_{j}\right), \frac{1}{2}\right)\right), f\left(E_{4}\left(\underline{v}_{F\left(e_{i}\right)}\left(x_{j}\right), \frac{1}{2}\right)\right)\right)$

$=M_{3}\left(f\left(E_{3}\left(\bar{v}_{F\left(e_{i}\right)}\left(x_{j}\right), \frac{1}{2}\right)\right), f\left(E_{4}\left(\underline{v}_{F\left(e_{i}\right)}\left(x_{j}\right), \frac{1}{2}\right)\right)\right.$,

$\left.f\left(E_{1}\left(\bar{u}_{F\left(e_{i}\right)}\left(x_{j}\right), \frac{1}{2}\right)\right), f\left(E_{2}\left(\underline{u}_{F\left(e_{i}\right)}\left(x_{j}\right), \frac{1}{2}\right)\right)\right)$

$=M_{3}\left(f\left(E_{1}\left(\bar{v}_{F\left(e_{i}\right)}\left(x_{j}\right), \frac{1}{2}\right)\right), f\left(E_{2}\left(\underline{v}_{F\left(e_{i}\right)}\left(x_{j}\right), \frac{1}{2}\right)\right)\right.$,

$\left.f\left(E_{3}\left(\bar{u}_{F\left(e_{i}\right)}\left(x_{j}\right), \frac{1}{2}\right)\right), f\left(E_{4}\left(\underline{u}_{F\left(e_{i}\right)}\left(x_{j}\right), \frac{1}{2}\right)\right)\right)$,

$\forall e_{i} \in P, x_{j} \in U$.

By Definition 16 we get

$D_{q}((F, P),(Q, P))=D_{q}\left(\left(F^{C}, P\right),(Q, P)\right)(q=1,2)$,
Thus, $I((F, P))=I\left((F, P)^{C}\right)$.

(4) Since $f^{\prime}$ is a fuzzy negation,

if $D_{q}((F, P),(Q, P)) \geqslant D_{q}((G, P),(Q, P))(q=1,2)$, then $f^{\prime}\left(2 D_{q}((F, P),(Q, P))\right) \leqslant f^{\prime}\left(2 D_{q}((G, P),(Q, P))\right)$ $(q=1,2)$, i.e., $I_{q}((F, P)) \leqslant I_{q}((G, P))(q=1,2)$.

Example 5. Now we list some aggregation operators $M_{3}$ which satisfy the conditions in Theorem 3: for any $x_{1}, x_{2}, x_{3}, x_{4} \in[0,1]$,

(1) $M_{3}\left(x_{1}, x_{2}, x_{3}, x_{4}\right)=\left(\frac{\left(x_{1} \vee x_{2}\right)^{p}+\left(x_{3} \vee x_{4}\right)^{p}}{2}\right)^{\frac{1}{p}}, p \geqslant 1$.

(2) $M_{3}\left(x_{1}, x_{2}, x_{3}, x_{4}\right)=\left(\frac{\left(x_{1}+x_{2}\right)^{p} \vee\left(x_{3}+x_{4}\right)^{p}}{2}\right)^{\frac{1}{p}}, p \geqslant 1$.

(3) $M_{3}\left(x_{1}, x_{2}, x_{3}, x_{4}\right)=\left(\frac{x_{1}^{p}+x_{2}^{p}+x_{3}^{p}+x_{4}^{p}}{4}\right)^{\frac{1}{p}}, p \geqslant 1$.

Theorem 4. Let $(Q, P)$ be an interval-valued intuitionistic fuzzy soft set on $U$, s.t. for any $e_{i} \in P$, $\left.Q\left(e_{i}\right)=\left\{\left\langle x_{j},[1 / 2,1 / 2]\right),[1 / 2,1 / 2]\right\rangle \mid x_{j} \in U\right\}$. Suppose that

(1) for each $p \in\{1,2\}, M_{p}$ is both a bottomaggregation operator and an idempotentaggregation operator;

(2) $M_{3}$ is both a top-aggregation operator and an idempotent-aggregation operator;

(3) $M_{3}\left(x_{1}, x_{2}, x_{3}, x_{4}\right)=M_{3}\left(x_{3}, x_{4}, x_{1}, x_{2}\right)$ for any $x_{1}, x_{2}, x_{3}, x_{4} \in[0,1]$;

(4) $E_{1}\left(x_{1}, x_{2}\right)=E_{2}\left(x_{1}, x_{2}\right)=E_{3}\left(x_{1}, x_{2}\right)=$ $E_{4}\left(x_{1}, x_{2}\right)=1-\left|x_{1}-x_{2}\right|$ for any $x_{1}, x_{2} \in[0,1]$;

(5) $f(x)=1-x$, for any $x \in[0,1]$;

(6) $D_{3}((F, P),(Q, P))$ and $D_{4}((F, P),(Q, P))$ are distance measures between $(F, P)$ and $(Q, P)$ given by Eq.(3) and Eq.(4) in Definition 16, respectively;

(7) $f^{\prime}$ is a strict fuzzy negation,

then for any $(F, P) \in I V I F S S(U)$,

$I_{q}((F, P))=f^{\prime}\left(2 D_{q}((F, P),(Q, P))\right)(q=3,4)$,

is an entropy for interval-valued intuitionistic fuzzy soft sets based on the corresponding distance $D_{q}$ $(q=3,4)$.

Theorem 5. Let $(Q, P)$ be an interval-valued intuitionistic fuzzy soft set on $U$, s.t. for any $e_{i} \in P$, $\left.Q\left(e_{i}\right)=\left\{\left\langle x_{j},[1 / 2,1 / 2]\right),[1 / 2,1 / 2]\right\rangle \mid x_{j} \in U\right\}$. Suppose that 
(1) $M_{1}$ is both a bottom-aggregation operator and an idempotent-aggregation operator;

(2) for each $p \in\{2,3\}, M_{p}$ is both a topaggregation operator and an idempotentaggregation operator;

(3) $M_{3}\left(x_{1}, x_{2}, x_{3}, x_{4}\right)=M_{3}\left(x_{3}, x_{4}, x_{1}, x_{2}\right)$ for any $x_{1}, x_{2}, x_{3}, x_{4} \in[0,1]$;

(4) $E_{1}\left(x_{1}, x_{2}\right)=E_{2}\left(x_{1}, x_{2}\right)=E_{3}\left(x_{1}, x_{2}\right)=$ $E_{4}\left(x_{1}, x_{2}\right)=1-\left|x_{1}-x_{2}\right|$ for any $x_{1}, x_{2} \in[0,1]$;

(5) $f(x)=1-x$, for any $x \in[0,1]$;

(6) $D_{5}((F, P),(Q, P))$ and $D_{6}((F, P),(Q, P))$ are distance measures between $(F, P)$ and $(Q, P)$ given by Eq.(5) and Eq.(6) in Definition 16, respectively;

(7) $f^{\prime}$ is a strict fuzzy negation,

then for any $(F, P) \in \operatorname{IVIFSS}(U)$, $I_{q}((F, P))=f^{\prime}\left(2 D_{q}((F, P),(Q, P))\right)(q=5,6)$,

is an entropy for interval-valued intuitionistic fuzzy soft sets based on the corresponding distance $D_{q}$ $(q=5,6)$.

Theorem 6. Let $(Q, P)$ be an interval-valued intuitionistic fuzzy soft set on $U$, s.t. for any $e_{i} \in P$, $\left.Q\left(e_{i}\right)=\left\{\left\langle x_{j},[1 / 2,1 / 2]\right),[1 / 2,1 / 2]\right\rangle \mid x_{j} \in U\right\}$. Suppose that

(1) for each $p \in\{1,2,3\}, \quad M_{p}$ is both an idempotent-aggregation operator and a topaggregation operator;

(2) $M_{3}\left(x_{1}, x_{2}, x_{3}, x_{4}\right)=M_{3}\left(x_{3}, x_{4}, x_{1}, x_{2}\right)$ for any $x_{1}, x_{2}, x_{3}, x_{4} \in[0,1]$;

(3) $E_{1}\left(x_{1}, x_{2}\right)=E_{2}\left(x_{1}, x_{2}\right)=E_{3}\left(x_{1}, x_{2}\right)=$ $E_{4}\left(x_{1}, x_{2}\right)=1-\left|x_{1}-x_{2}\right|$ for any $x_{1}, x_{2} \in[0,1]$;

(4) $f(x)=1-x$, for any $x \in[0,1]$;

(5) $D_{q}((F, P),(Q, P))(q=7,8)$ are distance measures between $(F, P)$ and $(Q, P)$ given by Eq.(7) and Eq.(8) in Definition 16, respectively;

(6) $f^{\prime}$ is a strict fuzzy negation, then for any $(F, P) \in I V I F S(U)$, $I_{q}((F, P))=f^{\prime}\left(2 D_{q}((F, P),(Q, P))\right)(q=7,8)$, is an entropy for interval-valued intuitiionistic fuzzy soft sets based on the corresponding distance $D_{q}$ $(q=7,8)$.

Example 6. Now we list some aggregation operators $M_{3}$ which satisfies the conditions in Theorem 4-6: for any $x_{1}, x_{2}, x_{3}, x_{4} \in[0,1]$,

(1) $M_{3}\left(x_{1}, x_{2}, x_{3}, x_{4}\right)=\left(\frac{\left(x_{1} \wedge x_{2}\right)^{p}+\left(x_{3} \wedge x_{4}\right)^{p}}{2}\right)^{\frac{1}{p}}, p \geqslant 1$.

(2) $M_{3}\left(x_{1}, x_{2}, x_{3}, x_{4}\right)=\left(\frac{\left(x_{1}+x_{2}\right)^{p} \wedge\left(x_{3}+x_{4}\right)^{p}}{2}\right)^{\frac{1}{p}}, p \geqslant 1$.

(3) $M_{3}\left(x_{1}, x_{2}, x_{3}, x_{4}\right)=\left(\frac{x_{1}^{p}+x_{2}^{p}+x_{3}^{p}+x_{4}^{p}}{4}\right)^{\frac{1}{p}}, p \geqslant 1$.

Remark 4. We can easily obtain a large number of distances by Definition 16, employing different aggregation operators. Furthermore, we can easily obtain a large number of entropies by Theorem 3-6, employing different distances.

\subsection{Transformation of entropies into similarity measures for IVIF SSs}

Next, we provide a transformational method of constructing similarity measure of IVIFSSs based on the entropy of IVIFSSs as below.

Definition 17. Let $(F, P),(G, P) \in \operatorname{IVIFSS}(U)$, assume that: for any $e_{i} \in P$,

$F\left(e_{i}\right)=\left\{\left\langle x_{j}, u_{F\left(e_{i}\right)}\left(x_{j}\right), v_{F\left(e_{i}\right)}\left(x_{j}\right)\right\rangle \mid x_{j} \in U\right\}=\left\{\left\langle x_{j}\right.\right.$, $\left.\left.\left[\underline{u}_{F\left(e_{i}\right)}\left(x_{j}\right), \bar{u}_{F\left(e_{i}\right)}\left(x_{j}\right)\right],\left[\underline{v}_{F\left(e_{i}\right)}\left(x_{j}\right), \bar{v}_{F\left(e_{i}\right)}\left(x_{j}\right)\right]\right\rangle \mid x_{j} \in U\right\}$,

$G\left(e_{i}\right)=\left\{\left\langle x_{j}, u_{G\left(e_{i}\right)}\left(x_{j}\right), v_{G\left(e_{i}\right)}\left(x_{j}\right)\right\rangle \mid x_{j} \in U\right\}=\left\{\left\langle x_{j}\right.\right.$, $\left.\left.\left[\underline{u}_{G\left(e_{i}\right)}\left(x_{j}\right), \bar{u}_{G\left(e_{i}\right)}\left(x_{j}\right)\right],\left[\underline{v}_{G\left(e_{i}\right)}\left(x_{j}\right), \bar{v}_{G\left(e_{i}\right)}\left(x_{j}\right)\right]\right\rangle \mid x_{j} \in U\right\}$.

Suppose that

(1) $M_{1}$ is a bottom-aggregation operator,

(2) $M_{1}\left(x_{1}, x_{2}, x_{3}, x_{4}\right) \geqslant M_{2}\left(x_{1}, x_{2}, x_{3}, x_{4}\right)$ for any $x_{1}, x_{2}, x_{3}, x_{4} \in[0,1]$

(3) $f$ is a strict fuzzy negation,

(4) $E_{l}(l=1,2,3,4)$ are fuzzy equivalence operators, 
then for any $\alpha \in[1,+\infty), \beta \in[1,+\infty)$, we can define a new interval-valued intuitionistic fuzzy set $\left(\psi_{1}(F, G), P\right)$ from $(F, P)$ and $(G, P)$ as follows: for any $e_{i} \in P, x_{j} \in U$,

$\underline{u}_{\psi_{1}(F, G)\left(e_{i}\right)}\left(x_{j}\right)=\frac{1}{2}\left\{1-\left[M_{1}\left(f\left(E_{1}\left(\underline{u}_{F\left(e_{i}\right)}\left(x_{j}\right), \underline{u}_{G\left(e_{i}\right)}\left(x_{j}\right)\right)\right)\right.\right.\right.$, $f\left(E_{2}\left(\bar{u}_{F\left(e_{i}\right)}\left(x_{j}\right), \bar{u}_{G\left(e_{i}\right)}\left(x_{j}\right)\right)\right), f\left(E_{3}\left(\underline{v}_{F\left(e_{i}\right)}\left(x_{j}\right), \underline{v}_{G\left(e_{i}\right)}\left(x_{j}\right)\right)\right)$, $\left.\left.\left.f\left(E_{4}\left(\bar{v}_{F\left(e_{i}\right)}\left(x_{j}\right), \bar{v}_{G\left(e_{i}\right)}\left(x_{j}\right)\right)\right)\right)\right]^{1 / \alpha}\right\}$;

$\bar{u}_{\psi_{1}(F, G)\left(e_{i}\right)}\left(x_{j}\right)=\frac{1}{2}\left\{1-\left[M_{1}\left(f\left(E_{1}\left(\underline{u}_{F\left(e_{i}\right)}\left(x_{j}\right), \underline{u}_{G\left(e_{i}\right)}\left(x_{j}\right)\right)\right)\right.\right.\right.$, $f\left(E_{2}\left(\bar{u}_{F\left(e_{i}\right)}\left(x_{j}\right), \bar{u}_{G\left(e_{i}\right)}\left(x_{j}\right)\right)\right), f\left(E_{3}\left(\underline{v}_{F\left(e_{i}\right)}\left(x_{j}\right), \underline{v}_{G\left(e_{i}\right)}\left(x_{j}\right)\right)\right)$, $\left.\left.\left.f\left(E_{4}\left(\bar{v}_{F\left(e_{i}\right)}\left(x_{j}\right), \bar{v}_{G\left(e_{i}\right)}\left(x_{j}\right)\right)\right)\right)\right]\right\}$;

$\underline{v}_{\psi_{1}(F, G)\left(e_{i}\right)}\left(x_{j}\right)=\frac{1}{2}\left\{1+\left[M_{2}\left(f\left(E_{1}\left(\underline{u}_{F\left(e_{i}\right)}\left(x_{j}\right), \underline{u}_{G\left(e_{i}\right)}\left(x_{j}\right)\right)\right)\right.\right.\right.$, $f\left(E_{2}\left(\bar{u}_{F\left(e_{i}\right)}\left(x_{j}\right), \bar{u}_{G\left(e_{i}\right)}\left(x_{j}\right)\right)\right), f\left(E_{3}\left(\underline{v}_{F\left(e_{i}\right)}\left(x_{j}\right), \underline{v}_{G\left(e_{i}\right)}\left(x_{j}\right)\right)\right)$, $\left.\left.\left.f\left(E_{4}\left(\bar{v}_{F\left(e_{i}\right)}\left(x_{j}\right), \bar{v}_{G\left(e_{i}\right)}\left(x_{j}\right)\right)\right)\right)\right]^{\beta}\right\}$;

$\bar{v}_{\psi_{1}(F, G)\left(e_{i}\right)}\left(x_{j}\right)=\frac{1}{2}\left\{1+\left[M_{2}\left(f\left(E_{1}\left(\underline{u}_{F\left(e_{i}\right)}\left(x_{j}\right), \underline{u}_{G\left(e_{i}\right)}\left(x_{j}\right)\right)\right)\right.\right.\right.$, $f\left(E_{2}\left(\bar{u}_{F\left(e_{i}\right)}\left(x_{j}\right), \bar{u}_{G\left(e_{i}\right)}\left(x_{j}\right)\right)\right), f\left(E_{3}\left(\underline{v}_{F\left(e_{i}\right)}\left(x_{j}\right), \underline{v}_{G\left(e_{i}\right)}\left(x_{j}\right)\right)\right)$, $\left.\left.\left.f\left(E_{4}\left(\bar{v}_{F\left(e_{i}\right)}\left(x_{j}\right), \bar{v}_{G\left(e_{i}\right)}\left(x_{j}\right)\right)\right)\right)\right]\right\}$.

Theorem 7. Let I be an entropy measure of interval-valued intuitionistic fuzzy soft set. For $(F, P),(G, P) \in \operatorname{IVIFSS}(U)$, then $I\left(\left(\psi_{1}(F, G), P\right)\right)$ is a similarity measure of $(F, P)$ and $(G, P)$.

Proof. We only need to prove that all the properties in Definition 10 hold.

(1)If $(F, P)$ is a classical soft set, then for all $e_{i} \in P, x_{j} \in U$, we know

$u_{F\left(e_{i}\right)}\left(x_{j}\right)=[1,1], v_{F\left(e_{i}\right)}\left(x_{j}\right)=[0,0], u_{F^{C}\left(e_{i}\right)}\left(x_{j}\right)=$ $[0,0], v_{F}{ }^{C}\left(e_{i}\right)\left(x_{j}\right)=[1,1]$, or

$u_{F\left(e_{i}\right)}\left(x_{j}\right)=[0,0], v_{F\left(e_{i}\right)}\left(x_{j}\right)=[1,1], u_{F^{C}\left(e_{i}\right)}\left(x_{j}\right)=$ $[1,1], v_{F^{C}\left(e_{i}\right)}\left(x_{j}\right)=[0,0]$,

then we have

$E_{1}\left(\underline{u}_{F\left(e_{i}\right)}\left(x_{j}\right), \underline{u}_{F^{C}\left(e_{i}\right)}\left(x_{j}\right)\right)=E_{2}\left(\bar{u}_{F\left(e_{i}\right)}\left(x_{j}\right), \bar{u}_{F^{C}\left(e_{i}\right)}\left(x_{j}\right)\right)=$

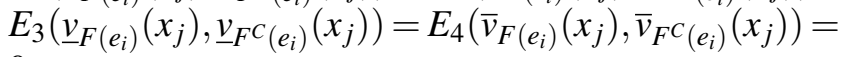
0 .

Since $M_{1}, M_{2}$ are aggregation operators, we get $M_{1}\left(f\left(E_{1}\left(\underline{u}_{F\left(e_{i}\right)}\left(x_{j}\right), \underline{u}_{F C}\left(e_{i}\right)\left(x_{j}\right)\right)\right), f\left(E_{2}\left(\bar{u}_{F\left(e_{i}\right)}\left(x_{j}\right)\right.\right.\right.$,

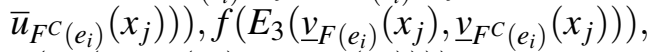
$\left.f\left(E_{4}\left(\bar{v}_{F\left(e_{i}\right)}\left(x_{j}\right), \bar{v}_{F}{ }^{C}\left(e_{i}\right)\left(x_{j}\right)\right)\right)\right)=1$,
$M_{2}\left(f\left(E_{1}\left(\underline{u}_{F\left(e_{i}\right)}\left(x_{j}\right), \underline{u}_{F C}\left(e_{i}\right)\left(x_{j}\right)\right)\right), f\left(E_{2}\left(\bar{u}_{F\left(e_{i}\right)}\left(x_{j}\right)\right.\right.\right.$, $\left.\left.\bar{u}_{F C\left(e_{i}\right)}\left(x_{j}\right)\right)\right), f\left(E_{3}\left(\underline{v}_{F\left(e_{i}\right)}\left(x_{j}\right), \underline{v}_{F}{ }^{C}\left(e_{i}\right)\left(x_{j}\right)\right)\right)$, $\left.f\left(E_{4}\left(\bar{v}_{F\left(e_{i}\right)}\left(x_{j}\right), \bar{v}_{F}\left(e_{i}\right)\left(x_{j}\right)\right)\right)\right)=1$, $\forall e_{i} \in P, x_{j} \in U$. hence we get

$\underline{u}_{\psi_{1}\left(F, F^{C}\right)\left(e_{i}\right)}\left(x_{j}\right)=\bar{u}_{\psi_{1}\left(F, F^{C}\right)\left(e_{i}\right)}\left(x_{j}\right)=0$,

$\underline{v}_{\psi_{1}\left(F, F^{C}\right)\left(e_{i}\right)}\left(x_{j}\right)=\bar{v}_{\psi_{1}\left(F, F^{C}\right)\left(e_{i}\right)}\left(x_{j}\right)=1$,

$\forall e_{i} \in P, x_{j} \in U$.

So, $\left(\psi_{1}\left(F, F^{C}\right), P\right)$ is crisp soft set in $U$.

By Definition 12 of entropy for IVIFSSs, we have $S\left((F, P),\left(F^{C}, P\right)\right)=I\left(\left(\psi_{1}\left(F, F^{C}\right), P\right)\right)=0$.

(2) $S((F, P),(G, P))=I\left(\left(\psi_{1}(F, G), P\right)\right)=1$.

$\Leftrightarrow u_{\psi_{1}(F, G)\left(e_{i}\right)}\left(x_{j}\right)=v_{\psi_{1}(F, G)\left(e_{i}\right)}\left(x_{j}\right)=\left[\frac{1}{2}, \frac{1}{2}\right]$,

$\forall e_{i} \in P, x_{j} \in U$.

$\Leftrightarrow M_{1}\left(f\left(E_{1}\left(\underline{u}_{F\left(e_{i}\right)}\left(x_{j}\right), \underline{u}_{G\left(e_{i}\right)}\left(x_{j}\right)\right)\right), f\left(E_{2}\left(\bar{u}_{F\left(e_{i}\right)}\left(x_{j}\right)\right.\right.\right.$, $\left.\left.\bar{u}_{G\left(e_{i}\right)}\left(x_{j}\right)\right)\right), f\left(E_{3}\left(\underline{v}_{F\left(e_{i}\right)}\left(x_{j}\right), \underline{v}_{G\left(e_{i}\right)}\left(x_{j}\right)\right)\right)$,

$\left.f\left(E_{4}\left(\bar{v}_{F\left(e_{i}\right)}\left(x_{j}\right), \bar{v}_{G\left(e_{i}\right)}\left(x_{j}\right)\right)\right)\right)=0$ and

$M_{2}\left(f\left(E_{1}\left(\underline{u}_{F\left(e_{i}\right)}\left(x_{j}\right), \underline{u}_{G\left(e_{i}\right)}\left(x_{j}\right)\right)\right), f\left(E_{2}\left(\bar{u}_{F\left(e_{i}\right)}\left(x_{j}\right)\right.\right.\right.$,

$\left.\left.\bar{u}_{G\left(e_{i}\right)}\left(x_{j}\right)\right)\right), f\left(E_{3}\left(\underline{v}_{F\left(e_{i}\right)}\left(x_{j}\right), \underline{v}_{G\left(e_{i}\right)}\left(x_{j}\right)\right)\right)$,

$\left.f\left(E_{4}\left(\bar{v}_{F\left(e_{i}\right)}\left(x_{j}\right), \bar{v}_{G\left(e_{i}\right)}\left(x_{j}\right)\right)\right)\right)=0$,

$\forall e_{i} \in P, \forall x_{j} \in U$.

$\Leftrightarrow f\left(E_{1}\left(\underline{u}_{F\left(e_{i}\right)}\left(x_{j}\right), \underline{u}_{G\left(e_{i}\right)}\left(x_{j}\right)\right)\right)=0$,

$f\left(E_{2}\left(\bar{u}_{F\left(e_{i}\right)}\left(x_{j}\right), \bar{u}_{G\left(e_{i}\right)}\left(x_{j}\right)\right)\right)=0$,

$f\left(E_{3}\left(\underline{v}_{F\left(e_{i}\right)}\left(x_{j}\right), \underline{v}_{G\left(e_{i}\right)}\left(x_{j}\right)\right)\right)=0$,

$f\left(E_{4}\left(\bar{v}_{F\left(e_{i}\right)}\left(x_{j}\right), \bar{v}_{G\left(e_{i}\right)}\left(x_{j}\right)\right)\right)=0$,

$\forall e_{i} \in P, \forall x_{j} \in U$.

$\Leftrightarrow E_{1}\left(\underline{u}_{F\left(e_{i}\right)}\left(x_{j}\right), \underline{u}_{G\left(e_{i}\right)}\left(x_{j}\right)\right)=E_{2}\left(\bar{u}_{F\left(e_{i}\right)}\left(x_{j}\right), \bar{u}_{G\left(e_{i}\right)}\left(x_{j}\right)\right)$ $=E_{3}\left(\underline{v}_{F\left(e_{i}\right)}\left(x_{j}\right), \underline{v}_{G\left(e_{i}\right)}\left(x_{j}\right)\right)=E_{4}\left(\bar{v}_{F\left(e_{i}\right)}\left(x_{j}\right), \bar{v}_{G\left(e_{i}\right)}\left(x_{j}\right)\right)$

$=1, \forall e_{i} \in P, \forall x_{j} \in U$.

$\Leftrightarrow \underline{u}_{F\left(e_{i}\right)}\left(x_{j}\right)=\underline{u}_{G\left(e_{i}\right)}\left(x_{j}\right), \bar{u}_{F\left(e_{i}\right)}\left(x_{j}\right)=\bar{u}_{G\left(e_{i}\right)}\left(x_{j}\right)$, $\underline{v}_{F\left(e_{i}\right)}\left(x_{j}\right)=\underline{v}_{G\left(e_{i}\right)}\left(x_{j}\right)$, and $\bar{v}_{F\left(e_{i}\right)}\left(x_{j}\right)=\bar{v}_{G\left(e_{i}\right)}\left(x_{j}\right)$,

$\forall e_{i} \in P, \forall x_{j} \in U$.

$\Leftrightarrow(F, P)=(G, P)$.

(3)From the definition of $\left(\psi_{1}(F, G), E\right)$, we know for any $e_{i} \in P, x_{j} \in U$,

$u_{\psi_{1}(F, G)\left(e_{i}\right)}\left(x_{j}\right)=u_{\psi_{1}(G, F)\left(e_{i}\right)}\left(x_{j}\right)$,

$v_{\psi_{1}(F, G)\left(e_{i}\right)}\left(x_{j}\right)=v_{\psi_{1}(G, F)\left(e_{i}\right)}\left(x_{j}\right)$,

that is, $\left(\psi_{1}(F, G), P\right)=\left(\psi_{1}(G, F), P\right)$,

then we get $I\left(\left(\psi_{1}(F, G), P\right)\right)=I\left(\left(\psi_{1}(G, F), P\right)\right)$

$\Leftrightarrow S((F, P),(G, P))=S((G, P),(F, P))$.

(4)If $(F, P) \subseteq(G, P) \subseteq(H, P)$, we know for any $e_{i} \in P, x_{j} \in U$,

$\underline{u}_{F\left(e_{i}\right)}\left(x_{j}\right) \leqslant \underline{u}_{G\left(e_{i}\right)}\left(x_{j}\right) \leqslant \underline{u}_{H\left(e_{i}\right)}\left(x_{j}\right)$,

$\bar{u}_{F\left(e_{i}\right)}\left(x_{j}\right) \leqslant \bar{u}_{G\left(e_{i}\right)}\left(x_{j}\right) \leqslant \bar{u}_{H\left(e_{i}\right)}\left(x_{j}\right)$,

$\underline{v}_{F\left(e_{i}\right)}\left(x_{j}\right) \geqslant \underline{v}_{G\left(e_{i}\right)}\left(x_{j}\right) \geqslant \underline{v}_{H\left(e_{i}\right)}\left(x_{j}\right)$,

$\bar{v}_{F\left(e_{i}\right)}\left(x_{j}\right) \geqslant \bar{v}_{G\left(e_{i}\right)}\left(x_{j}\right) \geqslant \bar{v}_{H\left(e_{i}\right)}\left(x_{j}\right)$, 
hence,

$E_{1}\left(\underline{u}_{F\left(e_{i}\right)}\left(x_{j}\right), \underline{u}_{H\left(e_{i}\right)}\left(x_{j}\right)\right) \leqslant E_{1}\left(\underline{u}_{F\left(e_{i}\right)}\left(x_{j}\right), \underline{u}_{G\left(e_{i}\right)}\left(x_{j}\right)\right)$, $E_{2}\left(\bar{u}_{F\left(e_{i}\right)}\left(x_{j}\right), \bar{u}_{H\left(e_{i}\right)}\left(x_{j}\right)\right) \leqslant E_{2}\left(\bar{u}_{F\left(e_{i}\right)}\left(x_{j}\right), \bar{u}_{G\left(e_{i}\right)}\left(x_{j}\right)\right)$, $E_{3}\left(\underline{v}_{F\left(e_{i}\right)}\left(x_{j}\right), \underline{v}_{H\left(e_{i}\right)}\left(x_{j}\right)\right) \leqslant E_{3}\left(\underline{v}_{F\left(e_{i}\right)}\left(x_{j}\right), \underline{v}_{G\left(e_{i}\right)}\left(x_{j}\right)\right)$, $E_{4}\left(\bar{v}_{F\left(e_{i}\right)}\left(x_{j}\right), \bar{v}_{H\left(e_{i}\right)}\left(x_{j}\right)\right) \leqslant E_{4}\left(\bar{v}_{F\left(e_{i}\right)}\left(x_{j}\right), \bar{v}_{G\left(e_{i}\right)}\left(x_{j}\right)\right)$, from properties of aggregation operators and decreasing monotone property of $f$ we have $M_{1}\left(f\left(E_{1}\left(\underline{u}_{F\left(e_{i}\right)}\left(x_{j}\right), \underline{u}_{H\left(e_{i}\right)}\left(x_{j}\right)\right)\right), f\left(E_{2}\left(\bar{u}_{F\left(e_{i}\right)}\left(x_{j}\right)\right.\right.\right.$, $\left.\left.\bar{u}_{H\left(e_{i}\right)}\left(x_{j}\right)\right)\right), f\left(E_{3}\left(\underline{v}_{F\left(e_{i}\right)}\left(x_{j}\right), \underline{v}_{H\left(e_{i}\right)}\left(x_{j}\right)\right)\right)$,

$\left.f\left(E_{4}\left(\bar{v}_{F\left(e_{i}\right)}\left(x_{j}\right), \bar{v}_{H\left(e_{i}\right)}\left(x_{j}\right)\right)\right)\right) \geqslant$

$M_{1}\left(f\left(E_{1}\left(\underline{u}_{F\left(e_{i}\right)}\left(x_{j}\right), \underline{u}_{G\left(e_{i}\right)}\left(x_{j}\right)\right)\right), f\left(E_{2}\left(\bar{u}_{F\left(e_{i}\right)}\left(x_{j}\right)\right.\right.\right.$, $\left.\left.\bar{u}_{G\left(e_{i}\right)}\left(x_{j}\right)\right)\right), f\left(E_{3}\left(\underline{v}_{F\left(e_{i}\right)}\left(x_{j}\right), \underline{v}_{G\left(e_{i}\right)}\left(x_{j}\right)\right)\right)$,

$\left.f\left(E_{4}\left(\bar{v}_{F\left(e_{i}\right)}\left(x_{j}\right), \bar{v}_{G\left(e_{i}\right)}\left(x_{j}\right)\right)\right)\right)$,

and

$M_{2}\left(f\left(E_{1}\left(\underline{u}_{F\left(e_{i}\right)}\left(x_{j}\right), \underline{u}_{H\left(e_{i}\right)}\left(x_{j}\right)\right)\right), f\left(E_{2}\left(\bar{u}_{F\left(e_{i}\right)}\left(x_{j}\right)\right.\right.\right.$,

$\left.\left.\bar{u}_{H\left(e_{i}\right)}\left(x_{j}\right)\right)\right), f\left(E_{3}\left(\underline{v}_{F\left(e_{i}\right)}\left(x_{j}\right), \underline{v}_{H\left(e_{i}\right)}\left(x_{j}\right)\right)\right)$,

$\left.f\left(E_{4}\left(\bar{v}_{F\left(e_{i}\right)}\left(x_{j}\right), \bar{v}_{H\left(e_{i}\right)}\left(x_{j}\right)\right)\right)\right) \geqslant$

$M_{2}\left(f\left(E_{1}\left(\underline{u}_{F\left(e_{i}\right)}\left(x_{j}\right), \underline{u}_{G\left(e_{i}\right)}\left(x_{j}\right)\right)\right), f\left(E_{2}\left(\bar{u}_{F\left(e_{i}\right)}\left(x_{j}\right)\right.\right.\right.$,

$\left.\left.\bar{u}_{G\left(e_{i}\right)}\left(x_{j}\right)\right)\right), f\left(E_{3}\left(\underline{v}_{F\left(e_{i}\right)}\left(x_{j}\right), \underline{v}_{G\left(e_{i}\right)}\left(x_{j}\right)\right)\right)$,

$\left.f\left(E_{4}\left(\bar{v}_{F\left(e_{i}\right)}\left(x_{j}\right), \bar{v}_{G\left(e_{i}\right)}\left(x_{j}\right)\right)\right)\right)$.

Thus, we get

$u_{\psi_{1}(F, H)\left(e_{i}\right)}\left(x_{j}\right) \leqslant u_{\psi_{1}(F, G)\left(e_{i}\right)}\left(x_{j}\right) \leqslant\left[\frac{1}{2}, \frac{1}{2}\right]$,

$v_{\psi_{1}(F, H)\left(e_{i}\right)}\left(x_{j}\right) \geqslant v_{\psi_{1}(F, G)\left(e_{i}\right)}\left(x_{j}\right) \geqslant\left[\frac{1}{2}, \frac{1}{2}\right]$,

$\forall e_{i} \in P, \forall x_{j} \in U$.

Let $(Q, P) \in \operatorname{IVIFSS}(U)$ and $Q\left(e_{i}\right)=$ $\left.\left\{\left\langle x_{j},[1 / 2,1 / 2]\right),[1 / 2,1 / 2]\right\rangle \mid x_{j} \in U\right\}$ for any $e_{i} \in P$, then we get

$\left(\psi_{1}(F, H), P\right) \subseteq\left(\psi_{1}(F, G), P\right) \subseteq(Q, P)$.

Similarly, we get

$\left(\psi_{1}(F, H), P\right) \subseteq\left(\psi_{1}(G, H), P\right) \subseteq(Q, P)$.

By Definition 9 of distance measure for IVIFSSs, we know

$D\left(\left(\psi_{1}(F, G), P\right),(Q, P)\right) \leqslant D\left(\left(\psi_{1}(F, H), P\right),(Q, P)\right)$,

$D\left(\left(\psi_{1}(G, H), P\right),(Q, P)\right) \leqslant D\left(\left(\psi_{1}(F, H), P\right),(Q, P)\right)$.

By Definition 12 of entropy for IVIFSSs, we conclude that

$I\left(\left(\psi_{1}(F, H), P\right)\right) \leqslant I\left(\left(\psi_{1}(F, G), P\right)\right)$,

$I\left(\left(\psi_{1}(F, H), P\right)\right) \leqslant I\left(\left(\psi_{1}(G, H), P\right)\right)$.

Hence,

$I\left(\left(\psi_{1}(F, H), P\right)\right) \leqslant I\left(\left(\psi_{1}(F, G), P\right)\right) \wedge I\left(\left(\psi_{1}(G, H), P\right)\right)$, that is,

$S((F, P),(H, P)) \leqslant S((F, P),(G, P)) \wedge S((G, P),(H, P))$.

Example 7. Let $I$ be an entropy measure of interval-valued intuitionistic fuzzy soft sets. For $(F, P),(G, P) \in \operatorname{IVIFSS}(U)$, assume that: for any $e_{i} \in P$,

$F\left(e_{i}\right)=\left\{\left\langle x_{j}, u_{F\left(e_{i}\right)}\left(x_{j}\right), v_{F\left(e_{i}\right)}\left(x_{j}\right)\right\rangle \mid x_{j} \in U\right\}=\left\{\left\langle x_{j}\right.\right.$, $\left.\left.\left[\underline{u}_{F\left(e_{i}\right)}\left(x_{j}\right), \bar{u}_{F\left(e_{i}\right)}\left(x_{j}\right)\right],\left[\underline{v}_{F\left(e_{i}\right)}\left(x_{j}\right), \bar{v}_{F\left(e_{i}\right)}\left(x_{j}\right)\right]\right\rangle \mid x_{j} \in U\right\}$,

$G\left(e_{i}\right)=\left\{\left\langle x_{j}, u_{G\left(e_{i}\right)}\left(x_{j}\right), v_{G\left(e_{i}\right)}\left(x_{j}\right)\right\rangle \mid x_{j} \in U\right\}=\left\{\left\langle x_{j}\right.\right.$, $\left.\left.\left[\underline{u}_{G\left(e_{i}\right)}\left(x_{j}\right), \bar{u}_{G\left(e_{i}\right)}\left(x_{j}\right)\right],\left[\underline{v}_{G\left(e_{i}\right)}\left(x_{j}\right), \bar{v}_{G\left(e_{i}\right)}\left(x_{j}\right)\right]\right\rangle \mid x_{j} \in U\right\}$. let

(1) $M_{1}\left(x_{1}, x_{2}, x_{3}, x_{4}\right)=\left(x_{1} \vee x_{2}\right) \vee\left(x_{3} \vee x_{4}\right)$,

(2) $M_{2}\left(x_{1}, x_{2}, x_{3}, x_{4}\right)=\left(x_{1} \vee x_{2}\right) \wedge\left(x_{3} \vee x_{4}\right)$,

(3) $E_{1}\left(x_{1}, x_{2}\right)=E_{2}\left(x_{1}, x_{2}\right)=E_{3}\left(x_{1}, x_{2}\right)=$ $E_{4}\left(x_{1}, x_{2}\right)=1-\left|x_{1}-x_{2}\right|$,

(4) $\alpha=\beta=2$,

(5) $f(x)=1-x$,

we get an interval-valued intuitionistic fuzzy soft set $\left(\psi_{1}^{\prime}(F, G), P\right)$ from $(F, P)$ and $(G, P)$ by Definition 17 as follows: for any $e_{i} \in P, x_{j} \in U$,

$\underline{u}_{\psi_{1}^{\prime}(F, G)\left(e_{i}\right)}\left(x_{j}\right)=\frac{1}{2}\left\{1-\left[\max \left(\left(\left|\underline{u}_{F\left(e_{i}\right)}\left(x_{j}\right)-\underline{u}_{G\left(e_{i}\right)}\left(x_{j}\right)\right|\right.\right.\right.\right.$ $\left.\vee\left|\bar{u}_{F\left(e_{i}\right)}\left(x_{j}\right)-\bar{u}_{G\left(e_{i}\right)}\left(x_{j}\right)\right|\right),\left(\left|\underline{v}_{F\left(e_{i}\right)}\left(x_{j}\right)-\underline{v}_{G\left(e_{i}\right)}\left(x_{j}\right)\right| \mathrm{V}\right.$ $\left.\left.\left.\left.\left|\bar{v}_{F\left(e_{i}\right)}\left(x_{j}\right)-\bar{v}_{G\left(e_{i}\right)}\left(x_{j}\right)\right|\right)\right)\right]^{1 / 2}\right\}$;

$\bar{u}_{\psi_{1}^{\prime}(F, G)\left(e_{i}\right)}\left(x_{j}\right)=\frac{1}{2}\left\{1-\left[\max \left(\left(\left|\underline{u}_{F\left(e_{i}\right)}\left(x_{j}\right)-\underline{u}_{G\left(e_{i}\right)}\left(x_{j}\right)\right|\right.\right.\right.\right.$ $\left.\vee\left|\bar{u}_{F\left(e_{i}\right)}\left(x_{j}\right)-\bar{u}_{G\left(e_{i}\right)}\left(x_{j}\right)\right|\right),\left(\left|\underline{v}_{F\left(e_{i}\right)}\left(x_{j}\right)-\underline{v}_{G\left(e_{i}\right)}\left(x_{j}\right)\right| \vee\right.$ $\left.\left.\left.\left.\left|\bar{v}_{F\left(e_{i}\right)}\left(x_{j}\right)-\bar{v}_{G\left(e_{i}\right)}\left(x_{j}\right)\right|\right)\right)\right]\right\}$;

$\underline{v}_{\psi_{1}^{\prime}(F, G)\left(e_{i}\right)}\left(x_{j}\right)=\frac{1}{2}\left\{1+\left[\min \left(\left(\left|\underline{u}_{F\left(e_{i}\right)}\left(x_{j}\right)-\underline{u}_{G\left(e_{i}\right)}\left(x_{j}\right)\right|\right.\right.\right.\right.$ $\left.\vee\left|\bar{u}_{F\left(e_{i}\right)}\left(x_{j}\right)-\bar{u}_{G\left(e_{i}\right)}\left(x_{j}\right)\right|\right),\left(\left|\underline{v}_{F\left(e_{i}\right)}\left(x_{j}\right)-\underline{v}_{G\left(e_{i}\right)}\left(x_{j}\right)\right| \mathrm{V}\right.$ $\left.\left.\left.\left.\left|\bar{v}_{F\left(e_{i}\right)}\left(x_{j}\right)-\bar{v}_{G\left(e_{i}\right)}\left(x_{j}\right)\right|\right)\right)\right]^{2}\right\}$;

$\bar{v}_{\psi_{1}^{\prime}(F, G)\left(e_{i}\right)}\left(x_{j}\right)=\frac{1}{2}\left\{1+\left[\min \left(\left(\left|\underline{u}_{F\left(e_{i}\right)}\left(x_{j}\right)-\underline{u}_{G\left(e_{i}\right)}\left(x_{j}\right)\right|\right.\right.\right.\right.$ $\left.\vee\left|\bar{u}_{F\left(e_{i}\right)}\left(x_{j}\right)-\bar{u}_{G\left(e_{i}\right)}\left(x_{j}\right)\right|\right),\left(\left|\underline{v}_{F\left(e_{i}\right)}\left(x_{j}\right)-\underline{v}_{G\left(e_{i}\right)}\left(x_{j}\right)\right|\right.$ $\left.\left.\left.\left.\vee\left|\bar{v}_{F\left(e_{i}\right)}\left(x_{j}\right)-\bar{v}_{G\left(e_{i}\right)}\left(x_{j}\right)\right|\right)\right)\right]\right\}$,

then $I\left(\left(\psi_{1}^{\prime}(F, G), P\right)\right)$ is a similarity measure of $(F, P)$ and $(G, P)$. 
Definition 18. Let $(F, P)$ and $(G, P)$ be two $\operatorname{IVIFSS}(U)$ in universe $U=\left\{x_{1}, x_{2}, \ldots x_{n}\right\}$, assume that: for any $e_{i} \in P$,

$F\left(e_{i}\right)=\left\{\left\langle x_{j}, u_{F\left(e_{i}\right)}\left(x_{j}\right), v_{F\left(e_{i}\right)}\left(x_{j}\right)\right\rangle \mid x_{j} \in U\right\}=\left\{\left\langle x_{j}\right.\right.$,

$\left.\left.\left[\underline{u}_{F\left(e_{i}\right)}\left(x_{j}\right), \bar{u}_{F\left(e_{i}\right)}\left(x_{j}\right)\right],\left[\underline{v}_{F\left(e_{i}\right)}\left(x_{j}\right), \bar{v}_{F\left(e_{i}\right)}\left(x_{j}\right)\right]\right\rangle \mid x_{j} \in U\right\}$,

$G\left(e_{i}\right)=\left\{\left\langle x_{j}, u_{G\left(e_{i}\right)}\left(x_{j}\right), v_{G\left(e_{i}\right)}\left(x_{j}\right)\right\rangle \mid x_{j} \in U\right\}=\left\{\left\langle x_{j}\right.\right.$,

$\left.\left.\left[\underline{u}_{G\left(e_{i}\right)}\left(x_{j}\right), \bar{u}_{G\left(e_{i}\right)}\left(x_{j}\right)\right],\left[\underline{v}_{G\left(e_{i}\right)}\left(x_{j}\right), \bar{v}_{G\left(e_{i}\right)}\left(x_{j}\right)\right]\right\rangle \mid x_{j} \in U\right\}$.

Suppose that $M_{1}, M_{2}$ are aggregation operators which satisfy that

(1) $M_{1}$ is a top-aggregation operator,

(2) $M_{1}\left(x_{1}, x_{2}, x_{3}, x_{4}\right) \leqslant M_{2}\left(x_{1}, x_{2}, x_{3}, x_{4}\right)$ for any $x_{1}, x_{2}, x_{3}, x_{4} \in[0,1]$

(3) $f$ is a strict fuzzy negation,

(4) $E_{l}(l=1,2,3,4)$ are fuzzy equivalence operators,

then for any $\alpha \in[1,+\infty), \beta \in[1,+\infty)$, we can define a new interval-valued intuitionistic fuzzy set $\left(\psi_{2}(F, G), P\right)$ from $(F, P)$ and $(G, P)$ as follows: for any $e_{i} \in P, x_{j} \in U$,

$\underline{u}_{\psi_{2}(F, G)\left(e_{i}\right)}\left(x_{j}\right)=\frac{1}{2}\left\{1-\left[f\left(M_{1}\left(E_{1}\left(\underline{u}_{F\left(e_{i}\right)}\left(x_{j}\right), \underline{u}_{G\left(e_{i}\right)}\left(x_{j}\right)\right)\right.\right.\right.\right.$,

$E_{2}\left(\bar{u}_{F\left(e_{i}\right)}\left(x_{j}\right), \bar{u}_{G\left(e_{i}\right)}\left(x_{j}\right)\right), E_{3}\left(\underline{v}_{F\left(e_{i}\right)}\left(x_{j}\right), \underline{v}_{G\left(e_{i}\right)}\left(x_{j}\right)\right)$,

$\left.\left.\left.\left.E_{4}\left(\bar{v}_{F\left(e_{i}\right)}\left(x_{j}\right), \bar{v}_{G\left(e_{i}\right)}\left(x_{j}\right)\right)\right)\right)\right]^{1 / \alpha}\right\}$;

$\bar{u}_{\psi_{2}(F, G)\left(e_{i}\right)}\left(x_{j}\right)=\frac{1}{2}\left\{1-\left[f\left(M_{1}\left(E_{1}\left(\underline{u}_{F\left(e_{i}\right)}\left(x_{j}\right), \underline{u}_{G\left(e_{i}\right)}\left(x_{j}\right)\right)\right.\right.\right.\right.$,

$E_{2}\left(\bar{u}_{F\left(e_{i}\right)}\left(x_{j}\right), \bar{u}_{G\left(e_{i}\right)}\left(x_{j}\right)\right), E_{3}\left(\underline{v}_{F\left(e_{i}\right)}\left(x_{j}\right), \underline{v}_{G\left(e_{i}\right)}\left(x_{j}\right)\right)$,

$\left.\left.\left.\left.E_{4}\left(\bar{v}_{F\left(e_{i}\right)}\left(x_{j}\right), \bar{v}_{G\left(e_{i}\right)}\left(x_{j}\right)\right)\right)\right)\right]\right\}$;

$\underline{v}_{\psi_{2}(F, G)\left(e_{i}\right)}\left(x_{j}\right)=\frac{1}{2}\left\{1+\left[f\left(M_{2}\left(E_{1}\left(\underline{u}_{F\left(e_{i}\right)}\left(x_{j}\right), \underline{u}_{G\left(e_{i}\right)}\left(x_{j}\right)\right)\right.\right.\right.\right.$,

$E_{2}\left(\bar{u}_{F\left(e_{i}\right)}\left(x_{j}\right), \bar{u}_{G\left(e_{i}\right)}\left(x_{j}\right)\right), E_{3}\left(\underline{v}_{F\left(e_{i}\right)}\left(x_{j}\right), \underline{v}_{G\left(e_{i}\right)}\left(x_{j}\right)\right)$,

$\left.\left.\left.\left.E_{4}\left(\bar{v}_{F\left(e_{i}\right)}\left(x_{j}\right), \bar{v}_{G\left(e_{i}\right)}\left(x_{j}\right)\right)\right)\right)\right]^{\beta}\right\}$;

$\bar{v}_{\psi_{2}(F, G)\left(e_{i}\right)}\left(x_{j}\right)=\frac{1}{2}\left\{1+\left[f\left(M_{2}\left(E_{1}\left(\underline{u}_{F\left(e_{i}\right)}\left(x_{j}\right), \underline{u}_{G\left(e_{i}\right)}\left(x_{j}\right)\right)\right.\right.\right.\right.$,

$E_{2}\left(\bar{u}_{F\left(e_{i}\right)}\left(x_{j}\right), \bar{u}_{G\left(e_{i}\right)}\left(x_{j}\right)\right), E_{3}\left(\underline{v}_{F\left(e_{i}\right)}\left(x_{j}\right), \underline{v}_{G\left(e_{i}\right)}\left(x_{j}\right)\right)$,

$\left.\left.\left.\left.E_{4}\left(\bar{v}_{F\left(e_{i}\right)}\left(x_{j}\right), \bar{v}_{G\left(e_{i}\right)}\left(x_{j}\right)\right)\right)\right)\right]\right\}$.
Theorem 8. Let I be an entropy measure of interval-valued intuitionistic fuzzy soft set. For $(F, P),(G, P) \in \operatorname{IVIFSS}(U)$, then $I\left(\left(\psi_{2}(F, G), P\right)\right)$ is a similarity measure of $(F, P)$ and $(G, P)$.

Example 8. Let $I$ be an entropy measure of interval-valued intuitionistic fuzzy soft sets. For $(F, P),(G, P) \in \operatorname{IVIFSS}(U)$, assume that: for any $e_{i} \in P$,

$F\left(e_{i}\right)=\left\{\left\langle x_{j}, u_{F\left(e_{i}\right)}\left(x_{j}\right), v_{F\left(e_{i}\right)}\left(x_{j}\right)\right\rangle \mid x_{j} \in U\right\}=\left\{\left\langle x_{j}\right.\right.$,

$\left.\left.\left[\underline{u}_{F\left(e_{i}\right)}\left(x_{j}\right), \bar{u}_{F\left(e_{i}\right)}\left(x_{j}\right)\right],\left[\underline{v}_{F\left(e_{i}\right)}\left(x_{j}\right), \bar{v}_{F\left(e_{i}\right)}\left(x_{j}\right)\right]\right\rangle \mid x_{j} \in U\right\}$,

$G\left(e_{i}\right)=\left\{\left\langle x_{j}, u_{G\left(e_{i}\right)}\left(x_{j}\right), v_{G\left(e_{i}\right)}\left(x_{j}\right)\right\rangle \mid x_{j} \in U\right\}=\left\{\left\langle x_{j}\right.\right.$,

$\left.\left.\left[\underline{u}_{G\left(e_{i}\right)}\left(x_{j}\right), \bar{u}_{G\left(e_{i}\right)}\left(x_{j}\right)\right],\left[\underline{v}_{G\left(e_{i}\right)}\left(x_{j}\right), \bar{v}_{G\left(e_{i}\right)}\left(x_{j}\right)\right]\right\rangle \mid x_{j} \in U\right\}$. let

(1) $M_{1}\left(x_{1}, x_{2}, x_{3}, x_{4}\right)=\frac{\left(x_{1}+x_{2}\right) \wedge\left(x_{3}+x_{4}\right)}{2}$ for any $x_{1}, x_{2}, x_{3}, x_{4} \in[0,1]$

(2) $M_{2}\left(x_{1}, x_{2}, x_{3}, x_{4}\right)=\frac{\left(x_{1}+x_{2}\right) \vee\left(x_{3}+x_{4}\right)}{2}$ for any $x_{1}, x_{2}, x_{3}, x_{4} \in[0,1]$

(3) $E_{1}\left(x_{1}, x_{2}\right)=E_{2}\left(x_{1}, x_{2}\right)=E_{3}\left(x_{1}, x_{2}\right)=$ $E_{4}\left(x_{1}, x_{2}\right)=\frac{2 x_{1} x_{2}}{x_{1}^{2}+x_{2}^{2}}$ for any $x_{1}, x_{2} \in[0,1]$.

(4) $\alpha=8, \beta=4, f(x)=1-x$,

we get an interval-valued intuitionistic fuzzy soft set $\left(\psi_{2}^{\prime}(F, G), P\right)$ from $(F, P)$ and $(G, P)$ by Definition 18 as follows: for any $e_{i} \in P, x_{j} \in U$,

$\underline{u}_{\psi_{1}^{\prime}(F, G)\left(e_{i}\right)}\left(x_{j}\right)=\frac{1}{2}\left\{1-\left[1-\frac{1}{2}\left(\frac{2 \underline{u}_{F\left(e_{i}\right)}\left(x_{j}\right) \underline{u}_{G\left(e_{i}\right)}\left(x_{j}\right)}{\underline{u}_{F\left(e_{i}\right)}\left(x_{j}\right)^{2}+\underline{u}_{G\left(e_{i}\right)}\left(x_{j}\right)^{2}}\right.\right.\right.$

$\left.+\frac{2 \bar{u}_{F\left(e_{i}\right)}\left(x_{j}\right) \bar{u}_{G\left(e_{i}\right)}\left(x_{j}\right)}{\bar{u}_{F\left(e_{i}\right)}\left(x_{j}\right)^{2}+\bar{u}_{G\left(e_{i}\right)}\left(x_{j}\right)^{2}}\right) \wedge\left(\frac{2 \underline{v}_{F\left(e_{i}\right)}\left(x_{j}\right) \underline{v}_{G\left(e_{i}\right)}\left(x_{j}\right)}{\underline{v}_{F\left(e_{i}\right)}\left(x_{j}\right)^{2}+\underline{v}_{G\left(e_{i}\right)}\left(x_{j}\right)^{2}}\right.$

$\left.\left.\left.+\frac{2 \bar{v}_{F\left(e_{i}\right)}\left(x_{j}\right) \bar{v}_{G\left(e_{i}\right)}\left(x_{j}\right)}{\bar{v}_{F\left(e_{i}\right)}\left(x_{j}\right)^{2}+\bar{v}_{G\left(e_{i}\right)}\left(x_{j}\right)^{2}}\right)\right]^{1 / 8}\right\}$

$\bar{u}_{\psi_{2}^{\prime}(F, G)\left(e_{i}\right)}\left(x_{j}\right)=\frac{1}{2}\left\{1-\left[1-\frac{1}{2}\left(\frac{2 \underline{u}_{F\left(e_{i}\right)}\left(x_{j}\right) \underline{u}_{G\left(e_{i}\right)}\left(x_{j}\right)}{\underline{u}_{F\left(e_{i}\right)}\left(x_{j}\right)^{2}+\underline{u}_{G\left(e_{i}\right)}\left(x_{j}\right)^{2}}\right.\right.\right.$

$\left.+\frac{2 \bar{u}_{F\left(e_{i}\right)}\left(x_{j}\right) \bar{u}_{G\left(e_{i}\right)}\left(x_{j}\right)}{\bar{u}_{F\left(e_{i}\right)}\left(x_{j}\right)^{2}+\bar{u}_{G\left(e_{i}\right)}\left(x_{j}\right)^{2}}\right) \wedge\left(\frac{2 \underline{v}_{F\left(e_{i}\right)}\left(x_{j}\right) \underline{v}_{G\left(e_{i}\right)}\left(x_{j}\right)}{\underline{v}_{F\left(e_{i}\right)}\left(x_{j}\right)^{2}+\underline{v}_{G\left(e_{i}\right)}\left(x_{j}\right)^{2}}\right.$

$\left.\left.\left.+\frac{2 \bar{v}_{F\left(e_{i}\right)}\left(x_{j}\right) \bar{v}_{G\left(e_{i}\right)}\left(x_{j}\right)}{\bar{v}_{F\left(e_{i}\right)}\left(x_{j}\right)^{2}+\bar{v}_{G\left(e_{i}\right)}\left(x_{j}\right)^{2}}\right)\right]\right\}$; 


$$
\begin{aligned}
& \underline{v}_{\psi_{2}^{\prime}(F, G)\left(e_{i}\right)}\left(x_{j}\right)=\frac{1}{2}\left\{1+\left[1-\frac{1}{2}\left(\frac{2 \underline{u}_{F\left(e_{i}\right)}\left(x_{j}\right) \underline{u}_{G\left(e_{i}\right)}\left(x_{j}\right)}{\underline{u}_{F\left(e_{i}\right)}\left(x_{j}\right)^{2}+\underline{u}_{G\left(e_{i}\right)}\left(x_{j}\right)^{2}}\right.\right.\right. \\
& \left.+\frac{2 \bar{u}_{F\left(e_{i}\right)}\left(x_{j}\right) \bar{u}_{G\left(e_{i}\right)}\left(x_{j}\right)}{\bar{u}_{F\left(e_{i}\right)}\left(x_{j}\right)^{2}+\bar{u}_{G\left(e_{i}\right)}\left(x_{j}\right)^{2}}\right) \vee\left(\frac{2 \underline{v}_{F\left(e_{i}\right)}\left(x_{j}\right) \underline{v}_{G\left(e_{i}\right)}\left(x_{j}\right)}{\underline{v}_{F\left(e_{i}\right)}\left(x_{j}\right)^{2}+\underline{v}_{G\left(e_{i}\right)}\left(x_{j}\right)^{2}}\right. \\
& \left.\left.\left.+\frac{2 \bar{v}_{F\left(e_{i}\right)}\left(x_{j}\right) \bar{v}_{G\left(e_{i}\right)}\left(x_{j}\right)}{\bar{v}_{F\left(e_{i}\right)}\left(x_{j}\right)^{2}+\bar{v}_{G\left(e_{i}\right)}\left(x_{j}\right)^{2}}\right)\right]^{4}\right\} ;
\end{aligned}
$$$$
\bar{v}_{\psi_{2}^{\prime}(F, G)\left(e_{i}\right)}\left(x_{j}\right)=\frac{1}{2}\left\{1+\left[1-\frac{1}{2}\left(\frac{2 \underline{u}_{F\left(e_{i}\right)}\left(x_{j}\right) \underline{u}_{G\left(e_{i}\right)}\left(x_{j}\right)}{\underline{u}_{F\left(e_{i}\right)}\left(x_{j}\right)^{2}+\underline{u}_{G\left(e_{i}\right)}\left(x_{j}\right)^{2}}\right.\right.\right.
$$$$
\left.+\frac{2 \bar{u}_{F\left(e_{i}\right)}\left(x_{j}\right) \bar{u}_{G\left(e_{i}\right)}\left(x_{j}\right)}{\bar{u}_{F\left(e_{i}\right)}\left(x_{j}\right)^{2}+\bar{u}_{G\left(e_{i}\right)}\left(x_{j}\right)^{2}}\right) \vee\left(\frac{2 \underline{v}_{F\left(e_{i}\right)}\left(x_{j}\right) \underline{v}_{G\left(e_{i}\right)}\left(x_{j}\right)}{\underline{v}_{F\left(e_{i}\right)}\left(x_{j}\right)^{2}+\underline{v}_{G\left(e_{i}\right)}\left(x_{j}\right)^{2}}\right.
$$$$
\left.\left.\left.+\frac{2 \bar{v}_{F\left(e_{i}\right)}\left(x_{j}\right) \bar{v}_{G\left(e_{i}\right)}\left(x_{j}\right)}{\bar{v}_{F\left(e_{i}\right)}\left(x_{j}\right)^{2}+\bar{v}_{G\left(e_{i}\right)}\left(x_{j}\right)^{2}}\right)\right]\right\},
$$

then $I\left(\left(\psi_{2}^{\prime}(F, G), P\right)\right)$ is a similarity measure of $(F, P)$ and $(G, P)$.

Definition 19. Let $(F, P),(G, P) \in \operatorname{IVIFSS}(U)$, assume that: for any $e_{i} \in P$,

$F\left(e_{i}\right)=\left\{\left\langle x_{j}, u_{F\left(e_{i}\right)}\left(x_{j}\right), v_{F\left(e_{i}\right)}\left(x_{j}\right)\right\rangle \mid x_{j} \in U\right\}=\left\{\left\langle x_{j}\right.\right.$,

$\left.\left.\left[\underline{u}_{F\left(e_{i}\right)}\left(x_{j}\right), \bar{u}_{F\left(e_{i}\right)}\left(x_{j}\right)\right],\left[\underline{v}_{F\left(e_{i}\right)}\left(x_{j}\right), \bar{v}_{F\left(e_{i}\right)}\left(x_{j}\right)\right]\right\rangle \mid x_{j} \in U\right\}$,

$G\left(e_{i}\right)=\left\{\left\langle x_{j}, u_{G\left(e_{i}\right)}\left(x_{j}\right), v_{G\left(e_{i}\right)}\left(x_{j}\right)\right\rangle \mid x_{j} \in U\right\}=\left\{\left\langle x_{j}\right.\right.$,

$\left.\left.\left[\underline{u}_{G\left(e_{i}\right)}\left(x_{j}\right), \bar{u}_{G\left(e_{i}\right)}\left(x_{j}\right)\right],\left[\underline{v}_{G\left(e_{i}\right)}\left(x_{j}\right), \bar{v}_{G\left(e_{i}\right)}\left(x_{j}\right)\right]\right\rangle \mid x_{j} \in U\right\}$.

Suppose that,

(1) $M$ is a top-aggregation operator,

(2) $f$ is a strict fuzzy negation,

(3) $E_{l}(l=1,2,3,4)$ are fuzzy equivalence operators,

then for $0<\alpha_{1} \leqslant \alpha_{2} \leqslant \alpha_{4} \leqslant \alpha_{3}$, we can define a new interval-valued intuitionistic fuzzy set $\left(\psi_{3}(F, G), P\right)$ from $(F, P)$ and $(G, P)$ as follows: for any $e_{i} \in P$, $x_{j} \in U$,

$$
\begin{aligned}
& \underline{u}_{\psi_{3}(F, G)\left(e_{i}\right)}\left(x_{j}\right)=\frac{1}{2}\left\{1-\left[f \left(M \left(E_{1}\left(\underline{u}_{F\left(e_{i}\right)}\left(x_{j}\right), \underline{u}_{G\left(e_{i}\right)}\left(x_{j}\right)\right),\right.\right.\right.\right. \\
& E_{2}\left(\bar{u}_{F\left(e_{i}\right)}\left(x_{j}\right), \bar{u}_{G\left(e_{i}\right)}\left(x_{j}\right)\right), E_{3}\left(\underline{v}_{F\left(e_{i}\right)}\left(x_{j}\right), \underline{v}_{G\left(e_{i}\right)}\left(x_{j}\right)\right), \\
& \left.\left.\left.\left.E_{4}\left(\bar{v}_{F\left(e_{i}\right)}\left(x_{j}\right), \bar{v}_{G\left(e_{i}\right)}\left(x_{j}\right)\right)\right)\right)\right]^{\alpha_{1}}\right\} ;
\end{aligned}
$$

$$
\begin{aligned}
& \bar{u}_{\psi_{3}(F, G)\left(e_{i}\right)}\left(x_{j}\right)=\frac{1}{2}\left\{1-\left[f \left(M \left(E_{1}\left(\underline{u}_{F\left(e_{i}\right)}\left(x_{j}\right), \underline{u}_{G\left(e_{i}\right)}\left(x_{j}\right)\right),\right.\right.\right.\right. \\
& E_{2}\left(\bar{u}_{F\left(e_{i}\right)}\left(x_{j}\right), \bar{u}_{G\left(e_{i}\right)}\left(x_{j}\right)\right), E_{3}\left(\underline{v}_{F\left(e_{i}\right)}\left(x_{j}\right), \underline{v}_{G\left(e_{i}\right)}\left(x_{j}\right)\right), \\
& \left.\left.\left.\left.E_{4}\left(\bar{v}_{F\left(e_{i}\right)}\left(x_{j}\right), \bar{v}_{G\left(e_{i}\right)}\left(x_{j}\right)\right)\right)\right)\right]^{\alpha_{2}}\right\} ; \\
& \underline{v}_{\psi_{3}(F, G)\left(e_{i}\right)}\left(x_{j}\right)=\frac{1}{2}\left\{1+\left[f \left(M \left(E_{1}\left(\underline{u}_{F\left(e_{i}\right)}\left(x_{j}\right), \underline{u}_{G\left(e_{i}\right)}\left(x_{j}\right)\right),\right.\right.\right.\right. \\
& E_{2}\left(\bar{u}_{F\left(e_{i}\right)}\left(x_{j}\right), \bar{u}_{G\left(e_{i}\right)}\left(x_{j}\right)\right), E_{3}\left(\underline{v}_{F\left(e_{i}\right)}\left(x_{j}\right), \underline{v}_{G\left(e_{i}\right)}\left(x_{j}\right)\right), \\
& \left.\left.\left.E_{4}\left(\bar{v}_{F\left(e_{i}\right)}\left(x_{j}\right), \bar{v}_{G\left(e_{i}\right)}\left(x_{j}\right)\right)\right)\right)\right]^{\left.\alpha_{3}\right\} ;} \\
& \bar{v}_{\psi_{3}(F, G)\left(e_{i}\right)}\left(x_{j}\right)=\frac{1}{2}\left\{1+\left[f \left(M \left(E_{1}\left(\underline{u}_{F\left(e_{i}\right)}\left(x_{j}\right), \underline{u}_{G\left(e_{i}\right)}\left(x_{j}\right)\right),\right.\right.\right.\right. \\
& E_{2}\left(\bar{u}_{F\left(e_{i}\right)}\left(x_{j}\right), \bar{u}_{G\left(e_{i}\right)}\left(x_{j}\right)\right), E_{3}\left(\underline{v}_{F\left(e_{i}\right)}\left(x_{j}\right), \underline{v}_{G\left(e_{i}\right)}\left(x_{j}\right)\right), \\
& \left.\left.\left.E_{4}\left(\bar{v}_{F\left(e_{i}\right)}\left(x_{j}\right), \bar{v}_{G\left(e_{i}\right)}\left(x_{j}\right)\right)\right)\right)\right]^{\left.\alpha_{4}\right\} .}
\end{aligned}
$$

Theorem 9. Let I be an entropy measure of interval-valued intuitionistic fuzzy soft set. For $(F, P),(G, P) \in \operatorname{IVIFSS}(U)$, then $I\left(\left(\psi_{3}(F, G), P\right)\right)$ is a similarity measure of $(F, P)$ and $(G, P)$.

Proof. We only need to prove that all the properties in Definition 10 hold.

(1)If $(F, P)$ is a classical soft set, then for $\forall e_{i} \in P$, $x_{j} \in U$, we know

$u_{F\left(e_{i}\right)}\left(x_{j}\right)=[1,1], v_{F\left(e_{i}\right)}\left(x_{j}\right)=[0,0], u_{F C}\left(e_{i}\right)\left(x_{j}\right)=$ $[0,0], v_{F C}\left(e_{i}\right)\left(x_{j}\right)=[1,1]$ or

$u_{F\left(e_{i}\right)}\left(x_{j}\right)=[0,0], v_{F\left(e_{i}\right)}\left(x_{j}\right)=[1,1], u_{F^{C}\left(e_{i}\right)}\left(x_{j}\right)=$ $[1,1], v_{F^{C}\left(e_{i}\right)}\left(x_{j}\right)=[0,0]$,

so we get

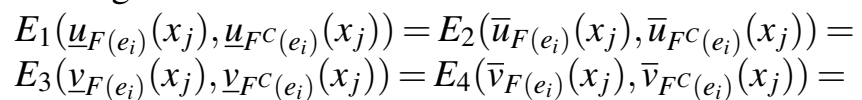
0 .

For $\forall \alpha_{i} \in\left\{\alpha_{1}, \alpha_{2}, \alpha_{3}, \alpha_{4}\right\}$ we have

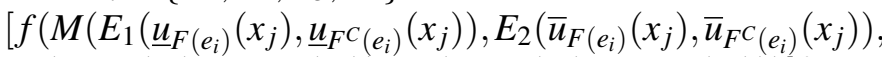

$\left.\left.E_{3}\left(\underline{v}_{F\left(e_{i}\right)}\left(x_{j}\right), \underline{v}_{F^{C}\left(e_{i}\right)}\left(x_{j}\right)\right), E_{4}\left(\bar{v}_{F\left(e_{i}\right)}\left(x_{j}\right), \bar{v}_{F}{ }^{C}\left(e_{i}\right)\left(x_{j}\right)\right)\right)\right]^{\alpha_{i}}=$ $1, \forall e_{i} \in P, x_{j} \in U$.

Hence, $\underline{u}_{\psi_{3}\left(F, F^{C}\right)\left(e_{i}\right)}\left(x_{j}\right)=\bar{u}_{\psi_{3}\left(F, F^{C}\right)\left(e_{i}\right)}\left(x_{j}\right)=0$,

$\underline{v}_{\psi_{3}\left(F, F^{C}\right)\left(e_{i}\right)}\left(x_{j}\right)=\bar{v}_{\psi_{3}\left(F, F^{C}\right)\left(e_{i}\right)}\left(x_{j}\right)=1$,

$\forall e_{i} \in P, x_{j} \in U$.

Thus, $\left(\psi_{3}\left(F, F^{C}\right), P\right)$ is classical soft set in $U$.

By Definition 12 of entropy for IVIFSSs, we have

$S\left((F, P),\left(F^{C}, P\right)\right)=I\left(\left(\psi_{3}\left(F, F^{C}\right), P\right)\right)=0$.

(2) $S((F, P),(G, P))=I\left(\left(\psi_{3}(F, G), P\right)\right)=1$

$\Leftrightarrow u_{\psi_{3}(F, G)\left(e_{i}\right)}\left(x_{j}\right)=v_{\psi_{3}(F, G)\left(e_{i}\right)}\left(x_{j}\right)=\left[\frac{1}{2}, \frac{1}{2}\right]$,

$\forall e_{i} \in P, x_{j} \in U$,

$\Leftrightarrow$ for $\forall \alpha_{i} \in\left\{\alpha_{1}, \alpha_{2}, \alpha_{3}, \alpha_{4}\right\}$,

$\left[f\left(M\left(E_{1}\left(\underline{u}_{F\left(e_{i}\right)}\left(x_{j}\right), \underline{u}_{G\left(e_{i}\right)}\left(x_{j}\right)\right), E_{2}\left(\bar{u}_{F\left(e_{i}\right)}\left(x_{j}\right), \bar{u}_{G\left(e_{i}\right)}\left(x_{j}\right)\right)\right.\right.\right.$, 
$\left.\left.\left.E_{3}\left(\underline{v}_{F\left(e_{i}\right)}\left(x_{j}\right), \underline{v}_{G\left(e_{i}\right)}\left(x_{j}\right)\right), E_{4}\left(\bar{v}_{F\left(e_{i}\right)}\left(x_{j}\right), \bar{v}_{G\left(e_{i}\right)}\left(x_{j}\right)\right)\right)\right)\right]^{\alpha_{i}}=$ $0, \forall e_{i} \in P, x_{j} \in U$,

$\Leftrightarrow M\left(E_{1}\left(\underline{u}_{F\left(e_{i}\right)}\left(x_{j}\right), \underline{u}_{G\left(e_{i}\right)}\left(x_{j}\right)\right), E_{2}\left(\bar{u}_{F\left(e_{i}\right)}\left(x_{j}\right), \bar{u}_{G\left(e_{i}\right)}\left(x_{j}\right)\right)\right.$, $\left.\left.E_{3}\left(\underline{v}_{F\left(e_{i}\right)}\left(x_{j}\right), \underline{v}_{G\left(e_{i}\right)}\left(x_{j}\right)\right), E_{4}\left(\bar{v}_{F\left(e_{i}\right)}\left(x_{j}\right), \bar{v}_{G\left(e_{i}\right)}\left(x_{j}\right)\right)\right)\right)=$ $1, \forall e_{i} \in P, x_{j} \in U$,

$\Leftrightarrow E_{1}\left(\underline{u}_{F\left(e_{i}\right)}\left(x_{j}\right), \underline{u}_{G\left(e_{i}\right)}\left(x_{j}\right)\right)=E_{2}\left(\bar{u}_{F\left(e_{i}\right)}\left(x_{j}\right), \bar{u}_{G\left(e_{i}\right)}\left(x_{j}\right)\right)=$ $E_{3}\left(\underline{v}_{F\left(e_{i}\right)}\left(x_{j}\right), \underline{v}_{G\left(e_{i}\right)}\left(x_{j}\right)\right)=E_{4}\left(\bar{v}_{F\left(e_{i}\right)}\left(x_{j}\right), \bar{v}_{G\left(e_{i}\right)}\left(x_{j}\right)\right)=$ $1, \forall e_{i} \in P, x_{j} \in U$,

$\Leftrightarrow \underline{u}_{F\left(e_{i}\right)}\left(x_{j}\right)=\underline{u}_{G\left(e_{i}\right)}\left(x_{j}\right), \bar{u}_{F\left(e_{i}\right)}\left(x_{j}\right)=\bar{u}_{G\left(e_{i}\right)}\left(x_{j}\right)$, $\underline{v}_{F\left(e_{i}\right)}\left(x_{j}\right)=\underline{v}_{G\left(e_{i}\right)}\left(x_{j}\right), \bar{v}_{F\left(e_{i}\right)}\left(x_{j}\right)=\bar{v}_{G\left(e_{i}\right)}\left(x_{j}\right)$,

$\forall e_{i} \in P, x_{j} \in U$,

$\Leftrightarrow(F, P)=(G, P)$.

(3)From the definition of $\left(\psi_{3}(F, G), P\right)$ we easily know that

$u_{\psi_{3}(F, G)\left(e_{i}\right)}\left(x_{j}\right)=u_{\psi_{3}(G, F)\left(e_{i}\right)}\left(x_{j}\right)$, $v_{\psi_{3}(F, G)\left(e_{i}\right)}\left(x_{j}\right)=v_{\psi_{3}(G, F)\left(e_{i}\right)}\left(x_{j}\right)$,

i.e. $\left(\psi_{3}(F, G), P\right)=\left(\psi_{3}(G, F), P\right)$.

Thus, $I\left(\left(\psi_{3}(F, G), P\right)\right)=I\left(\left(\psi_{3}(G, F), P\right)\right)$

$\Leftrightarrow S((F, P),(G, P))=S((G, P),(F, P))$.

(4)If $(F, P) \subseteq(G, P) \subseteq(H, P)$, then we know

$\underline{u}_{F\left(e_{i}\right)}\left(x_{j}\right) \leqslant \underline{u}_{G\left(e_{i}\right)}\left(x_{j}\right) \leqslant \underline{u}_{H\left(e_{i}\right)}\left(x_{j}\right)$,

$\bar{u}_{F\left(e_{i}\right)}\left(x_{j}\right) \leqslant \bar{u}_{G\left(e_{i}\right)}\left(x_{j}\right) \leqslant \bar{u}_{H\left(e_{i}\right)}\left(x_{j}\right)$,

$\underline{v}_{F\left(e_{i}\right)}\left(x_{j}\right) \geqslant \underline{v}_{G\left(e_{i}\right)}\left(x_{j}\right) \geqslant \underline{v}_{H\left(e_{i}\right)}\left(x_{j}\right)$,

$\bar{v}_{F\left(e_{i}\right)}\left(x_{j}\right) \geqslant \bar{v}_{G\left(e_{i}\right)}\left(x_{j}\right) \geqslant \bar{v}_{H\left(e_{i}\right)}\left(x_{j}\right)$,

$\forall e_{i} \in P, x_{j} \in U$,

hence,

$E_{1}\left(\underline{u}_{F\left(e_{i}\right)}\left(x_{j}\right), \underline{u}_{H\left(e_{i}\right)}\left(x_{j}\right)\right) \leqslant E_{1}\left(\underline{u}_{F\left(e_{i}\right)}\left(x_{j}\right), \underline{u}_{G\left(e_{i}\right)}\left(x_{j}\right)\right)$, $E_{2}\left(\bar{u}_{F\left(e_{i}\right)}\left(x_{j}\right), \bar{u}_{H\left(e_{i}\right)}\left(x_{j}\right)\right) \leqslant E_{2}\left(\bar{u}_{F\left(e_{i}\right)}\left(x_{j}\right), \bar{u}_{G\left(e_{i}\right)}\left(x_{j}\right)\right)$, $E_{3}\left(\underline{v}_{F\left(e_{i}\right)}\left(x_{j}\right), \underline{v}_{H\left(e_{i}\right)}\left(x_{j}\right)\right) \leqslant E_{3}\left(\underline{v}_{F\left(e_{i}\right)}\left(x_{j}\right), \underline{v}_{G\left(e_{i}\right)}\left(x_{j}\right)\right)$, $E_{4}\left(\bar{v}_{F\left(e_{i}\right)}\left(x_{j}\right), \bar{v}_{H\left(e_{i}\right)}\left(x_{j}\right)\right) \leqslant E_{4}\left(\bar{v}_{F\left(e_{i}\right)}\left(x_{j}\right), \bar{v}_{G\left(e_{i}\right)}\left(x_{j}\right)\right)$, $\forall e_{i} \in P, x_{j} \in U$,

then we have, for $\forall \alpha_{i} \in\left\{\alpha_{1}, \alpha_{2}, \alpha_{3}, \alpha_{4}\right\}$,

$\left[f\left(M\left(E_{1}\left(\underline{u}_{F\left(e_{i}\right)}\left(x_{j}\right), \underline{u}_{H\left(e_{i}\right)}\left(x_{j}\right)\right), E_{2}\left(\bar{u}_{F\left(e_{i}\right)}\left(x_{j}\right), \bar{u}_{H\left(e_{i}\right)}\left(x_{j}\right)\right)\right.\right.\right.$, $\left.\left.\left.E_{3}\left(\underline{v}_{F\left(e_{i}\right)}\left(x_{j}\right), \underline{v}_{H\left(e_{i}\right)}\left(x_{j}\right)\right), E_{4}\left(\bar{v}_{F\left(e_{i}\right)}\left(x_{j}\right), \bar{v}_{H\left(e_{i}\right)}\left(x_{j}\right)\right)\right)\right)\right]^{\alpha_{i}} \geqslant$ $\left[f\left(M\left(E_{1}\left(\underline{u}_{F\left(e_{i}\right)}\left(x_{j}\right), \underline{u}_{G\left(e_{i}\right)}\left(x_{j}\right)\right), E_{2}\left(\bar{u}_{F\left(e_{i}\right)}\left(x_{j}\right), \bar{u}_{G\left(e_{i}\right)}\left(x_{j}\right)\right)\right.\right.\right.$, $\left.\left.\left.E_{3}\left(\underline{v}_{F\left(e_{i}\right)}\left(x_{j}\right), \underline{v}_{G\left(e_{i}\right)}\left(x_{j}\right)\right), E_{4}\left(\bar{v}_{F\left(e_{i}\right)}\left(x_{j}\right), \bar{v}_{G\left(e_{i}\right)}\left(x_{j}\right)\right)\right)\right)\right]^{\alpha_{i}}$, $\forall e_{i} \in P, x_{j} \in U$,

so we get,

$u_{\psi_{3}(F, H)\left(e_{i}\right)}\left(x_{j}\right) \leqslant u_{\psi_{3}(F, G)\left(e_{i}\right)}\left(x_{j}\right) \leqslant\left[\frac{1}{2}, \frac{1}{2}\right]$,

$v_{\psi_{3}(F, H)\left(e_{i}\right)}\left(x_{j}\right) \geqslant v_{\psi_{3}(F, G)\left(e_{i}\right)}\left(x_{j}\right) \geqslant\left[\frac{1}{2}, \frac{1}{2}\right]$,

$\forall e_{i} \in P, \forall x_{j} \in U$.

Let $(Q, P) \in \operatorname{IVIFSS}(U)$ and $Q\left(e_{i}\right)=$ $\left.\left\{\left\langle x_{j},[1 / 2,1 / 2]\right),[1 / 2,1 / 2]\right\rangle \mid x_{j} \in U\right\}$ for any $e_{i} \in P$, then we get

$\left(\psi_{3}(F, H), P\right) \subseteq\left(\psi_{3}(F, G), P\right) \subseteq(Q, P)$.

Similarly, we get
$\left(\psi_{3}(F, H), P\right) \subseteq\left(\psi_{3}(G, H), P\right) \subseteq(Q, P)$.

By Definition 9 of distance measure for IVIFSSs, we know

$D\left(\left(\psi_{3}(F, G), P\right),(Q, P)\right) \leqslant D\left(\left(\psi_{3}(F, H), P\right),(Q, P)\right)$,

$D\left(\left(\psi_{3}(G, H), P\right),(Q, P)\right) \leqslant D\left(\left(\psi_{3}(F, H), P\right),(Q, P)\right)$.

By Definition 12 of entropy for IVIFSSs, we conclude that

$I\left(\left(\psi_{3}(F, H), P\right)\right) \leqslant I\left(\left(\psi_{3}(F, G), P\right)\right)$,

$I\left(\left(\psi_{3}(F, H), P\right)\right) \leqslant I\left(\left(\psi_{3}(G, H), P\right)\right)$.

Hence,

$I\left(\left(\psi_{3}(F, H), P\right)\right) \leqslant I\left(\left(\psi_{3}(F, G), P\right)\right) \wedge I\left(\left(\psi_{3}(G, H), P\right)\right)$, that is,

$S((F, P),(H, P)) \leqslant S((F, P),(G, P)) \wedge S((G, P),(H, P))$.

Definition 20. Let $(F, P),(G, P) \in \operatorname{IVIFSS}(U)$, assume that: for any $e_{i} \in P$,

$F\left(e_{i}\right)=\left\{\left\langle x_{j}, u_{F\left(e_{i}\right)}\left(x_{j}\right), v_{F\left(e_{i}\right)}\left(x_{j}\right)\right\rangle \mid x_{j} \in U\right\}=\left\{\left\langle x_{j}\right.\right.$,

$\left.\left.\left[\underline{u}_{F\left(e_{i}\right)}\left(x_{j}\right), \bar{u}_{F\left(e_{i}\right)}\left(x_{j}\right)\right],\left[\underline{v}_{F\left(e_{i}\right)}\left(x_{j}\right), \bar{v}_{F\left(e_{i}\right)}\left(x_{j}\right)\right]\right\rangle \mid x_{j} \in U\right\}$,

$G\left(e_{i}\right)=\left\{\left\langle x_{j}, u_{G\left(e_{i}\right)}\left(x_{j}\right), v_{G\left(e_{i}\right)}\left(x_{j}\right)\right\rangle \mid x_{j} \in U\right\}=\left\{\left\langle x_{j}\right.\right.$,

$\left.\left.\left[\underline{u}_{G\left(e_{i}\right)}\left(x_{j}\right), \bar{u}_{G\left(e_{i}\right)}\left(x_{j}\right)\right],\left[\underline{v}_{G\left(e_{i}\right)}\left(x_{j}\right), \bar{v}_{G\left(e_{i}\right)}\left(x_{j}\right)\right]\right\rangle \mid x_{j} \in U\right\}$.

Suppose that,

(1) $M$ is a bottom-aggregation operator,

(2) $f$ is a strict fuzzy negation,

(3) $E_{l}(l=1,2,3,4)$ are fuzzy equivalence operators,

then for $0<\alpha_{1} \leqslant \alpha_{2} \leqslant \alpha_{4} \leqslant \alpha_{3}$, we can define a new interval-valued intuitionistic fuzzy set $\left(\psi_{4}(F, G), P\right)$ from $(F, P)$ and $(G, P)$ as follows: for any $e_{i} \in P$, $x_{j} \in U$,

$\underline{u}_{\psi_{4}(F, G)\left(e_{i}\right)}\left(x_{j}\right)=\frac{1}{2}\left\{1-\left[M\left(f\left(E_{1}\left(\underline{u}_{F\left(e_{i}\right)}\left(x_{j}\right), \underline{u}_{G\left(e_{i}\right)}\left(x_{j}\right)\right)\right)\right.\right.\right.$, $f\left(E_{2}\left(\bar{u}_{F\left(e_{i}\right)}\left(x_{j}\right), \bar{u}_{G\left(e_{i}\right)}\left(x_{j}\right)\right)\right), f\left(E_{3}\left(\underline{v}_{F\left(e_{i}\right)}\left(x_{j}\right), \underline{v}_{G\left(e_{i}\right)}\left(x_{j}\right)\right)\right)$, $\left.\left.\left.f\left(E_{4}\left(\bar{v}_{F\left(e_{i}\right)}\left(x_{j}\right), \bar{v}_{G\left(e_{i}\right)}\left(x_{j}\right)\right)\right)\right)\right]^{\alpha_{1}}\right\}$;

$\bar{u}_{\psi_{4}(F, G)\left(e_{i}\right)}\left(x_{j}\right)=\frac{1}{2}\left\{1-\left[M\left(f\left(E_{1}\left(\underline{u}_{F\left(e_{i}\right)}\left(x_{j}\right), \underline{u}_{G\left(e_{i}\right)}\left(x_{j}\right)\right)\right)\right.\right.\right.$, $f\left(E_{2}\left(\bar{u}_{F\left(e_{i}\right)}\left(x_{j}\right), \bar{u}_{G\left(e_{i}\right)}\left(x_{j}\right)\right)\right), f\left(E_{3}\left(\underline{v}_{F\left(e_{i}\right)}\left(x_{j}\right), \underline{v}_{G\left(e_{i}\right)}\left(x_{j}\right)\right)\right)$, $\left.\left.\left.f\left(E_{4}\left(\bar{v}_{F\left(e_{i}\right)}\left(x_{j}\right), \bar{v}_{G\left(e_{i}\right)}\left(x_{j}\right)\right)\right)\right)\right]^{\alpha_{2}}\right\} ;$ 
$\underline{v}_{\psi_{4}(F, G)\left(e_{i}\right)}\left(x_{j}\right)=\frac{1}{2}\left\{1+\left[M\left(f\left(E_{1}\left(\underline{u}_{F\left(e_{i}\right)}\left(x_{j}\right), \underline{u}_{G\left(e_{i}\right)}\left(x_{j}\right)\right)\right)\right.\right.\right.$, $f\left(E_{2}\left(\bar{u}_{F\left(e_{i}\right)}\left(x_{j}\right), \bar{u}_{G\left(e_{i}\right)}\left(x_{j}\right)\right)\right), f\left(E_{3}\left(\underline{v}_{F\left(e_{i}\right)}\left(x_{j}\right), \underline{v}_{G\left(e_{i}\right)}\left(x_{j}\right)\right)\right)$, $\left.\left.\left.f\left(E_{4}\left(\bar{v}_{F\left(e_{i}\right)}\left(x_{j}\right), \bar{v}_{G\left(e_{i}\right)}\left(x_{j}\right)\right)\right)\right)\right]^{\alpha_{3}}\right\}$;

$\bar{v}_{\psi_{4}(F, G)\left(e_{i}\right)}\left(x_{j}\right)=\frac{1}{2}\left\{1+\left[M\left(f\left(E_{1}\left(\underline{u}_{F\left(e_{i}\right)}\left(x_{j}\right), \underline{u}_{G\left(e_{i}\right)}\left(x_{j}\right)\right)\right)\right.\right.\right.$, $f\left(E_{2}\left(\bar{u}_{F\left(e_{i}\right)}\left(x_{j}\right), \bar{u}_{G\left(e_{i}\right)}\left(x_{j}\right)\right)\right), f\left(E_{3}\left(\underline{v}_{F\left(e_{i}\right)}\left(x_{j}\right), \underline{v}_{G\left(e_{i}\right)}\left(x_{j}\right)\right)\right)$, $\left.\left.\left.f\left(E_{4}\left(\bar{v}_{F\left(e_{i}\right)}\left(x_{j}\right), \bar{v}_{G\left(e_{i}\right)}\left(x_{j}\right)\right)\right)\right)\right]^{\alpha_{4}}\right\}$.

Theorem 10. Let I be an entropy measure of interval-valued intuitionistic fuzzy soft set. For $(F, P),(G, P) \in \operatorname{IVIFSS}(U)$, then $I\left(\left(\psi_{4}(F, G), P\right)\right)$ is a similarity measure of $(F, P)$ and $(G, P)$.

Example 9. Let $I$ be an entropy measure of interval-valued intuitionistic fuzzy soft sets. For $(F, P),(G, P) \in \operatorname{IVIFSS}(U)$, for any $e_{i} \in P$,

$F\left(e_{i}\right)=\left\{\left\langle x_{j}, u_{F\left(e_{i}\right)}\left(x_{j}\right), v_{F\left(e_{i}\right)}\left(x_{j}\right)\right\rangle \mid x_{j} \in U\right\}=\left\{\left\langle x_{j}\right.\right.$,

$\left.\left.\left[\underline{u}_{F\left(e_{i}\right)}\left(x_{j}\right), \bar{u}_{F\left(e_{i}\right)}\left(x_{j}\right)\right],\left[\underline{v}_{F\left(e_{i}\right)}\left(x_{j}\right), \bar{v}_{F\left(e_{i}\right)}\left(x_{j}\right)\right]\right\rangle \mid x_{j} \in U\right\}$,

$G\left(e_{i}\right)=\left\{\left\langle x_{j}, u_{G\left(e_{i}\right)}\left(x_{j}\right), v_{G\left(e_{i}\right)}\left(x_{j}\right)\right\rangle \mid x_{j} \in U\right\}=\left\{\left\langle x_{j}\right.\right.$, $\left.\left.\left[\underline{u}_{G\left(e_{i}\right)}\left(x_{j}\right), \bar{u}_{G\left(e_{i}\right)}\left(x_{j}\right)\right],\left[\underline{v}_{G\left(e_{i}\right)}\left(x_{j}\right), \bar{v}_{G\left(e_{i}\right)}\left(x_{j}\right)\right]\right\rangle \mid x_{j} \in U\right\}$.

Let

(1) $M\left(x_{1}, x_{2}, x_{3}, x_{4}\right)=\frac{x_{1}+x_{2}+x_{3}+x_{4}}{4}$ for any $x_{1}, x_{2}, x_{3}, x_{4} \in[0,1]$,

(2) $E_{1}\left(x_{1}, x_{2}\right)=E_{2}\left(x_{1}, x_{2}\right)=E_{3}\left(x_{1}, x_{2}\right)=$ $E_{4}\left(x_{1}, x_{2}\right)=1-\left|x_{1}-x_{2}\right|$ for any $x_{1}, x_{2} \in[0,1]$,

(3) $\alpha_{1}=2, \alpha_{2}=3, \alpha_{3}=5, \alpha_{4}=4$,

(4) $f(x)=1-x$,

we get an interval-valued intuitionistic fuzzy soft set $\left(\psi_{4}^{\prime}(F, G), P\right)$ from $(F, P)$ and $(G, P)$ by Definition 20 as follows: for any $e_{i} \in P, x_{j} \in U$,

$$
\begin{aligned}
& \underline{u}_{\psi_{4}^{\prime}(F, G)\left(e_{i}\right)}\left(x_{j}\right)=\frac{1}{2}\left\{1-\left[\frac { 1 } { 4 } \left(\left|\underline{u}_{F\left(e_{i}\right)}\left(x_{j}\right)-\underline{u}_{G\left(e_{i}\right)}\left(x_{j}\right)\right|\right.\right.\right. \\
& +\left|\bar{u}_{F\left(e_{i}\right)}\left(x_{j}\right)-\bar{u}_{G\left(e_{i}\right)}\left(x_{j}\right)\right|+\left|\underline{v}_{F\left(e_{i}\right)}\left(x_{j}\right)-\underline{v}_{G\left(e_{i}\right)}\left(x_{j}\right)\right| \\
& \left.\left.\left.+\left|\bar{v}_{F\left(e_{i}\right)}\left(x_{j}\right)-\bar{v}_{G\left(e_{i}\right)}\left(x_{j}\right)\right|\right)\right]^{2}\right\} ; \\
& \bar{u}_{\psi_{4}^{\prime}(F, G)\left(e_{i}\right)}\left(x_{j}\right)=\frac{1}{2}\left\{1-\left[\frac { 1 } { 4 } \left(\left|\underline{u}_{F\left(e_{i}\right)}\left(x_{j}\right)-\underline{u}_{G\left(e_{i}\right)}\left(x_{j}\right)\right|\right.\right.\right. \\
& +\left|\bar{u}_{F\left(e_{i}\right)}\left(x_{j}\right)-\bar{u}_{G\left(e_{i}\right)}\left(x_{j}\right)\right|+\left|\underline{v}_{F\left(e_{i}\right)}\left(x_{j}\right)-\underline{v}_{G\left(e_{i}\right)}\left(x_{j}\right)\right| \\
& \left.\left.\left.+\left|\bar{v}_{F\left(e_{i}\right)}\left(x_{j}\right)-\bar{v}_{G\left(e_{i}\right)}\left(x_{j}\right)\right|\right)\right]^{3}\right\} ;
\end{aligned}
$$

$$
\begin{aligned}
& \underline{v}_{\psi_{4}^{\prime}(F, G)\left(e_{i}\right)}\left(x_{j}\right)=\frac{1}{2}\left\{1+\left[\frac { 1 } { 4 } \left(\left|\underline{u}_{F\left(e_{i}\right)}\left(x_{j}\right)-\underline{u}_{G\left(e_{i}\right)}\left(x_{j}\right)\right|\right.\right.\right. \\
& +\left|\bar{u}_{F\left(e_{i}\right)}\left(x_{j}\right)-\bar{u}_{G\left(e_{i}\right)}\left(x_{j}\right)\right|+\left|\underline{v}_{F\left(e_{i}\right)}\left(x_{j}\right)-\underline{v}_{G\left(e_{i}\right)}\left(x_{j}\right)\right| \\
& \left.\left.\left.+\left|\bar{v}_{F\left(e_{i}\right)}\left(x_{j}\right)-\bar{v}_{G\left(e_{i}\right)}\left(x_{j}\right)\right|\right)\right]^{5}\right\} ; \\
& \bar{v}_{\psi_{4}^{\prime}(F, G)\left(e_{i}\right)}\left(x_{j}\right)=\frac{1}{2}\left\{1+\left[\frac { 1 } { 4 } \left(\left|\underline{u}_{F\left(e_{i}\right)}\left(x_{j}\right)-\underline{u}_{G\left(e_{i}\right)}\left(x_{j}\right)\right|\right.\right.\right. \\
& +\left|\bar{u}_{F\left(e_{i}\right)}\left(x_{j}\right)-\bar{u}_{G\left(e_{i}\right)}\left(x_{j}\right)\right|+\left|\underline{v}_{F\left(e_{i}\right)}\left(x_{j}\right)-\underline{v}_{G\left(e_{i}\right)}\left(x_{j}\right)\right| \\
& \left.\left.\left.+\left|\bar{v}_{F\left(e_{i}\right)}\left(x_{j}\right)-\bar{v}_{G\left(e_{i}\right)}\left(x_{j}\right)\right|\right)\right]^{4}\right\},
\end{aligned}
$$

then $I\left(\left(\psi_{4}^{\prime}(F, G), P\right)\right)$ is a similarity measure of $(F, P)$ and $(G, P)$.

Theorem 11. If I is an entropy measure of IVIFSSs and $\left(\psi_{h}(F, G), P\right)(h=1,2,3,4)$ is given by Definition 17-20, then $I\left(\left(\psi_{h}(F, G)^{C}, P\right)\right)(h=1,2,3,4)$ is also a similarity measure between $(F, P)$ and $(G, P)$.

Remark 5. Based on Definition 17-20, by selecting different aggregation operators and fuzzy equivalences, we can obtain a large number of IVIFSSs, which can be used to transform an entropy measure into a similarity measure for IVIFSSs.

Remark 6. If $(F, P),(G, P) \in I V I F S S(U)$ degenerate to $F, G \in I V I F S(U)$, the specific interval-valued intuitionistic fuzzy soft set $\left(\psi_{1}^{\prime}(F, G), P\right)$ in Example 7 degenerates to $\psi_{1}^{\prime}(F, G) \in I V I F S(U)$. The entropy of $\psi_{1}^{\prime}(F, G)$ has been proven a similarity measure between $F$ and $G$ in Ref. ${ }^{17}$. Our research in this subsection can be regarded as a generalization and extension of the research in Ref. ${ }^{17}$ based on fuzzy equivalences and aggregation operators. However, even if it degenerates to the IVIFSs situation, all the formulae given by Definition 18-20 in this work are new.

\subsection{Transformation of entropies into inclusion measures for IVIF SSS}

Definition 21. Let $(F, P),(G, P) \in I V I F S S(U)$, assume that: for any $e_{i} \in P$,

$$
\begin{aligned}
& F\left(e_{i}\right)=\left\{\left\langle x_{j}, u_{F\left(e_{i}\right)}\left(x_{j}\right), v_{F\left(e_{i}\right)}\left(x_{j}\right)\right\rangle \mid x_{j} \in U\right\}=\left\{\left\langlex_{j},\right.\right. \\
& \left.\left.\left[\underline{u}_{F\left(e_{i}\right)}\left(x_{j}\right), \bar{u}_{F\left(e_{i}\right)}\left(x_{j}\right)\right],\left[\underline{v}_{F\left(e_{i}\right)}\left(x_{j}\right), \bar{v}_{F\left(e_{i}\right)}\left(x_{j}\right)\right]\right\rangle \mid x_{j} \in U\right\}, \\
& G\left(e_{i}\right)=\left\{\left\langle x_{j}, u_{G\left(e_{i}\right)}\left(x_{j}\right), v_{G\left(e_{i}\right)}\left(x_{j}\right)\right\rangle \mid x_{j} \in U\right\}=\left\{\left\langlex_{j},\right.\right. \\
& \left.\left.\left[\underline{u}_{G\left(e_{i}\right)}\left(x_{j}\right), \bar{u}_{G\left(e_{i}\right)}\left(x_{j}\right)\right],\left[\underline{v}_{G\left(e_{i}\right)}\left(x_{j}\right), \bar{v}_{G\left(e_{i}\right)}\left(x_{j}\right)\right]\right\rangle \mid x_{j} \in U\right\} .
\end{aligned}
$$


Suppose that,

(1) $M_{1}$ is a bottom-aggregation operator,

(2) $M_{1}\left(x_{1}, x_{2}, x_{3}, x_{4}\right) \geqslant M_{2}\left(x_{1}, x_{2}, x_{3}, x_{4}\right)$ for any $x_{1}, x_{2}, x_{3}, x_{4} \in[0,1]$,

(3) $f$ is a strict fuzzy negation,

(4) $E_{l}(l=1,2,3,4)$ are fuzzy equivalence operators,

then for any $\alpha \in[1,+\infty), \beta \in[1,+\infty)$, we can define a new interval-valued intuitionistic fuzzy set $\left(\phi_{1}(F, G), P\right)$ from $(F, P)$ and $(G, P)$ as follows: for any $e_{i} \in P, x_{j} \in U$,

$\underline{u}_{\phi_{1}(F, G)\left(e_{i}\right)}\left(x_{j}\right)=\frac{1}{2}\left\{1-\left[M_{1}\left(f\left(E_{1}\left(\underline{u}_{F\left(e_{i}\right)}\left(x_{j}\right), \underline{u}_{F\left(e_{i}\right)}\left(x_{j}\right)\right.\right.\right.\right.\right.$

$\left.\left.\wedge \underline{u}_{G\left(e_{i}\right)}\left(x_{j}\right)\right)\right), f\left(E_{2}\left(\bar{u}_{F\left(e_{i}\right)}\left(x_{j}\right), \bar{u}_{F\left(e_{i}\right)}\left(x_{j}\right) \wedge \bar{u}_{G\left(e_{i}\right)}\left(x_{j}\right)\right)\right)$, $f\left(E_{3}\left(\underline{v}_{F\left(e_{i}\right)}\left(x_{j}\right), \underline{v}_{F\left(e_{i}\right)}\left(x_{j}\right) \vee \underline{v}_{G\left(e_{i}\right)}\left(x_{j}\right)\right)\right), f\left(E_{4}\left(\bar{v}_{F\left(e_{i}\right)}\left(x_{j}\right)\right.\right.$, $\left.\left.\left.\left.\left.\bar{v}_{F\left(e_{i}\right)}\left(x_{j}\right) \vee \bar{v}_{G\left(e_{i}\right)}\left(x_{j}\right)\right)\right)\right)\right]^{1 / \alpha}\right\}$;

$\bar{u}_{\phi_{1}(F, G)\left(e_{i}\right)}\left(x_{j}\right)=\frac{1}{2}\left\{1-\left[M_{1}\left(f\left(E_{1}\left(\underline{u}_{F\left(e_{i}\right)}\left(x_{j}\right), \underline{u}_{F\left(e_{i}\right)}\left(x_{j}\right)\right.\right.\right.\right.\right.$ $\left.\left.\wedge \underline{u}_{G\left(e_{i}\right)}\left(x_{j}\right)\right)\right), f\left(E_{2}\left(\bar{u}_{F\left(e_{i}\right)}\left(x_{j}\right), \bar{u}_{F\left(e_{i}\right)}\left(x_{j}\right) \wedge \bar{u}_{G\left(e_{i}\right)}\left(x_{j}\right)\right)\right)$, $f\left(E_{3}\left(\underline{v}_{F\left(e_{i}\right)}\left(x_{j}\right), \underline{v}_{F\left(e_{i}\right)}\left(x_{j}\right) \vee \underline{v}_{G\left(e_{i}\right)}\left(x_{j}\right)\right)\right), f\left(E_{4}\left(\bar{v}_{F\left(e_{i}\right)}\left(x_{j}\right)\right.\right.$, $\left.\left.\left.\left.\left.\bar{v}_{F\left(e_{i}\right)}\left(x_{j}\right) \vee \bar{v}_{G\left(e_{i}\right)}\left(x_{j}\right)\right)\right)\right)\right]\right\}$;

$\underline{v}_{\phi_{1}(F, G)\left(e_{i}\right)}\left(x_{j}\right)=\frac{1}{2}\left\{1+\left[M_{2}\left(f\left(E_{1}\left(\underline{u}_{F\left(e_{i}\right)}\left(x_{j}\right), \underline{u}_{F\left(e_{i}\right)}\left(x_{j}\right)\right.\right.\right.\right.\right.$ $\left.\left.\wedge \underline{u}_{G\left(e_{i}\right)}\left(x_{j}\right)\right)\right), f\left(E_{2}\left(\bar{u}_{F\left(e_{i}\right)}\left(x_{j}\right), \bar{u}_{F\left(e_{i}\right)}\left(x_{j}\right) \wedge \bar{u}_{G\left(e_{i}\right)}\left(x_{j}\right)\right)\right)$, $f\left(E_{3}\left(\underline{v}_{F\left(e_{i}\right)}\left(x_{j}\right), \underline{v}_{F\left(e_{i}\right)}\left(x_{j}\right) \vee \underline{v}_{G\left(e_{i}\right)}\left(x_{j}\right)\right)\right), f\left(E_{4}\left(\bar{v}_{F\left(e_{i}\right)}\left(x_{j}\right)\right.\right.$, $\left.\left.\left.\left.\left.\bar{v}_{F\left(e_{i}\right)}\left(x_{j}\right) \vee \bar{v}_{G\left(e_{i}\right)}\left(x_{j}\right)\right)\right)\right)\right]^{\beta}\right\}$;

$\bar{v}_{\phi_{1}(F, G)\left(e_{i}\right)}\left(x_{j}\right)=\frac{1}{2}\left\{1+\left[M_{2}\left(f\left(E_{1}\left(\underline{u}_{F\left(e_{i}\right)}\left(x_{j}\right), \underline{u}_{F\left(e_{i}\right)}\left(x_{j}\right)\right.\right.\right.\right.\right.$ $\left.\left.\wedge \underline{u}_{G\left(e_{i}\right)}\left(x_{j}\right)\right)\right), f\left(E_{2}\left(\bar{u}_{F\left(e_{i}\right)}\left(x_{j}\right), \bar{u}_{F\left(e_{i}\right)}\left(x_{j}\right) \wedge \bar{u}_{G\left(e_{i}\right)}\left(x_{j}\right)\right)\right)$, $f\left(E_{3}\left(\underline{v}_{F\left(e_{i}\right)}\left(x_{j}\right), \underline{v}_{F\left(e_{i}\right)}\left(x_{j}\right) \vee \underline{v}_{G\left(e_{i}\right)}\left(x_{j}\right)\right)\right), f\left(E_{4}\left(\bar{v}_{F\left(e_{i}\right)}\left(x_{j}\right)\right.\right.$, $\left.\left.\left.\left.\left.\bar{v}_{F\left(e_{i}\right)}\left(x_{j}\right) \vee \bar{v}_{G\left(e_{i}\right)}\left(x_{j}\right)\right)\right)\right)\right]\right\}$.

Theorem 12. Let I be an entropy measure of interval-valued intuitionistic fuzzy soft set. For $(F, P),(G, P) \in \operatorname{IVIFSS}(U)$, then $I\left(\left(\phi_{1}(F, G), P\right)\right)$ is an inclusion measure between $(F, P)$ and $(G, P)$.
Proof. We only need to prove that all the properties in Definition 11 hold.

(1) If $(F, P)=(U, P),(G, P)=(\emptyset, P)$, we get $F\left(e_{i}\right)=\left\{\left\langle x_{j},[1,1],[0,0]\right\rangle \mid x_{j} \in U\right\}, G\left(e_{i}\right)=$ $\left\{\left\langle x_{j},[0,0],[1,1]\right\rangle \mid x_{j} \in U\right\}$ for $\forall e_{i} \in P$, then we have for any $x_{j} \in U, e_{i} \in P$

$E_{1}\left(\underline{u}_{F\left(e_{i}\right)}\left(x_{j}\right), \underline{u}_{F\left(e_{i}\right)}\left(x_{j}\right) \wedge \underline{u}_{G\left(e_{i}\right)}\left(x_{j}\right)\right)=E_{1}(1,1 \wedge 0)=$ 0 ,

$E_{2}\left(\bar{u}_{F\left(e_{i}\right)}\left(x_{j}\right), \bar{u}_{F\left(e_{i}\right)}\left(x_{j}\right) \wedge \bar{u}_{G\left(e_{i}\right)}\left(x_{j}\right)\right)=E_{2}(1,1 \wedge 0)=$ 0 ,

$E_{3}\left(\underline{v}_{F\left(e_{i}\right)}\left(x_{j}\right), \underline{v}_{F\left(e_{i}\right)}\left(x_{j}\right) \vee \underline{v}_{G\left(e_{i}\right)}\left(x_{j}\right)\right)=E_{3}(0,0 \vee 1)=$ 0 ,

$E_{4}\left(\underline{v}_{F\left(e_{i}\right)}\left(x_{j}\right), \underline{v}_{F\left(e_{i}\right)}\left(x_{j}\right) \vee \underline{v}_{G\left(e_{i}\right)}\left(x_{j}\right)\right)=E_{4}(0,0 \vee 1)=$ 0 ,

so we get

$M_{1}\left(f\left(E_{1}\left(\underline{u}_{F\left(e_{i}\right)}\left(x_{j}\right), \underline{u}_{F\left(e_{i}\right)}\left(x_{j}\right) \wedge \underline{u}_{G\left(e_{i}\right)}\left(x_{j}\right)\right)\right)\right.$,

$f\left(E_{2}\left(\bar{u}_{F\left(e_{i}\right)}\left(x_{j}\right), \bar{u}_{F\left(e_{i}\right)}\left(x_{j}\right) \wedge \bar{u}_{G\left(e_{i}\right)}\left(x_{j}\right)\right)\right)$,

$f\left(E_{3}\left(\underline{v}_{F\left(e_{i}\right)}\left(x_{j}\right), \underline{v}_{F\left(e_{i}\right)}\left(x_{j}\right) \vee \underline{v}_{G\left(e_{i}\right)}\left(x_{j}\right)\right)\right)$,

$\left.f\left(E_{4}\left(\bar{v}_{F\left(e_{i}\right)}\left(x_{j}\right), \bar{v}_{F\left(e_{i}\right)}\left(x_{j}\right) \vee \bar{v}_{G\left(e_{i}\right)}\left(x_{j}\right)\right)\right)\right)$

$=M_{1}(1,1,1,1)=1$ and

$M_{2}\left(f\left(E_{1}\left(\underline{u}_{F\left(e_{i}\right)}\left(x_{j}\right), \underline{u}_{F\left(e_{i}\right)}\left(x_{j}\right) \wedge \underline{u}_{G\left(e_{i}\right)}\left(x_{j}\right)\right)\right)\right.$,

$f\left(E_{2}\left(\bar{u}_{F\left(e_{i}\right)}\left(x_{j}\right), \bar{u}_{F\left(e_{i}\right)}\left(x_{j}\right) \wedge \bar{u}_{G\left(e_{i}\right)}\left(x_{j}\right)\right)\right)$,

$f\left(E_{3}\left(\underline{v}_{F\left(e_{i}\right)}\left(x_{j}\right), \underline{v}_{F\left(e_{i}\right)}\left(x_{j}\right) \vee \underline{v}_{G\left(e_{i}\right)}\left(x_{j}\right)\right)\right)$,

$\left.f\left(E_{4}\left(\bar{v}_{F\left(e_{i}\right)}\left(x_{j}\right), \bar{v}_{F\left(e_{i}\right)}\left(x_{j}\right) \vee \bar{v}_{G\left(e_{i}\right)}\left(x_{j}\right)\right)\right)\right)$

$=M_{2}(1,1,1,1)=1$.

Thus, it is easy to get that

$\left[\underline{u}_{\phi_{1}(F, G)\left(e_{i}\right)}\left(x_{j}\right), \bar{u}_{\phi_{1}(F, G)\left(e_{i}\right)}\left(x_{j}\right)\right]=[0,0]$,

$\left[\underline{v}_{\phi_{1}(F, G)\left(e_{i}\right)}\left(x_{j}\right), \bar{v}_{\phi_{1}(F, G)\left(e_{i}\right)}\left(x_{j}\right)\right]=[1,1]$,

$\forall x_{j} \in U, e_{i} \in P$.

By Definition 12 of entropy for IVIFSSs, we know $I\left(\left(\phi_{1}(F, G), P\right)\right)=0 \Leftrightarrow J((F, P),(G, P))=0$.

(2) $I\left(\left(\phi_{1}(F, G), P\right)\right)=J((F, P),(G, P))=1$,

$\Leftrightarrow\left[\underline{u}_{\phi_{1}(F, G)\left(e_{i}\right)}\left(x_{j}\right), \bar{u}_{\phi_{1}(F, G)\left(e_{i}\right)}\left(x_{j}\right)\right]=\left[\frac{1}{2}, \frac{1}{2}\right]$,

$\left[\underline{v}_{\phi_{1}(F, G)\left(e_{i}\right)}\left(x_{j}\right), \bar{v}_{\phi_{1}(F, G)\left(e_{i}\right)}\left(x_{j}\right)\right]=\left[\frac{1}{2}, \frac{1}{2}\right]$,

$\forall x_{j} \in U, e_{i} \in P$.

$\Leftrightarrow M_{1}\left(f\left(E_{1}\left(\underline{u}_{F\left(e_{i}\right)}\left(x_{j}\right), \underline{u}_{F\left(e_{i}\right)}\left(x_{j}\right) \wedge \underline{u}_{G\left(e_{i}\right)}\left(x_{j}\right)\right)\right)\right.$,

$f\left(E_{2}\left(\bar{u}_{F\left(e_{i}\right)}\left(x_{j}\right), \bar{u}_{F\left(e_{i}\right)}\left(x_{j}\right) \wedge \bar{u}_{G\left(e_{i}\right)}\left(x_{j}\right)\right)\right)$,

$f\left(E_{3}\left(\underline{v}_{F\left(e_{i}\right)}\left(x_{j}\right), \underline{v}_{F\left(e_{i}\right)}\left(x_{j}\right) \vee \underline{v}_{G\left(e_{i}\right)}\left(x_{j}\right)\right)\right)$,

$\left.f\left(E_{4}\left(\bar{v}_{F\left(e_{i}\right)}\left(x_{j}\right), \bar{v}_{F\left(e_{i}\right)}\left(x_{j}\right) \vee \bar{v}_{G\left(e_{i}\right)}\left(x_{j}\right)\right)\right)\right)=0$,

and

$M_{2}\left(f\left(E_{1}\left(\underline{u}_{F\left(e_{i}\right)}\left(x_{j}\right), \underline{u}_{F\left(e_{i}\right)}\left(x_{j}\right) \wedge \underline{u}_{G\left(e_{i}\right)}\left(x_{j}\right)\right)\right)\right.$,

$f\left(E_{2}\left(\bar{u}_{F\left(e_{i}\right)}\left(x_{j}\right), \bar{u}_{F\left(e_{i}\right)}\left(x_{j}\right) \wedge \bar{u}_{G\left(e_{i}\right)}\left(x_{j}\right)\right)\right)$,

$f\left(E_{3}\left(\underline{v}_{F\left(e_{i}\right)}\left(x_{j}\right), \underline{v}_{F\left(e_{i}\right)}\left(x_{j}\right) \vee \underline{v}_{G\left(e_{i}\right)}\left(x_{j}\right)\right)\right)$,

$\left.f\left(E_{4}\left(\bar{v}_{F\left(e_{i}\right)}\left(x_{j}\right), \bar{v}_{F\left(e_{i}\right)}\left(x_{j}\right) \vee \bar{v}_{G\left(e_{i}\right)}\left(x_{j}\right)\right)\right)\right)=0$,

$\forall x_{j} \in U, e_{i} \in P$.

$\Leftrightarrow f\left(E_{1}\left(\underline{u}_{F\left(e_{i}\right)}\left(x_{j}\right), \underline{u}_{F\left(e_{i}\right)}\left(x_{j}\right) \wedge \underline{u}_{G\left(e_{i}\right)}\left(x_{j}\right)\right)\right)=0$, 
$f\left(E_{2}\left(\bar{u}_{F\left(e_{i}\right)}\left(x_{j}\right), \bar{u}_{F\left(e_{i}\right)}\left(x_{j}\right) \wedge \bar{u}_{G\left(e_{i}\right)}\left(x_{j}\right)\right)\right)=0$,
$f\left(E_{3}\left(\underline{v}_{F\left(e_{i}\right)}\left(x_{j}\right), \underline{v}_{F\left(e_{i}\right)}\left(x_{j}\right) \vee \underline{v}_{G\left(e_{i}\right)}\left(x_{j}\right)\right)\right)=0$,
$f\left(E_{4}\left(\bar{v}_{F\left(e_{i}\right)}\left(x_{j}\right), \bar{v}_{F\left(e_{i}\right)}\left(x_{j}\right) \vee \bar{v}_{G\left(e_{i}\right)}\left(x_{j}\right)\right)=0\right.$,
$\forall x_{j} \in U, e_{i} \in P$.
$\Leftrightarrow E_{1}\left(\underline{u}_{F\left(e_{i}\right)}\left(x_{j}\right), \underline{u}_{F\left(e_{i}\right)}\left(x_{j}\right) \wedge \underline{u}_{G\left(e_{i}\right)}\left(x_{j}\right)\right)=1$,
$E_{2}\left(\bar{u}_{F\left(e_{i}\right)}\left(x_{j}\right), \bar{u}_{F\left(e_{i}\right)}\left(x_{j}\right) \wedge \bar{u}_{G\left(e_{i}\right)}\left(x_{j}\right)\right)=1$,
$E_{3}\left(\underline{v}_{F\left(e_{i}\right)}\left(x_{j}\right), \underline{v}_{F\left(e_{i}\right)}\left(x_{j}\right) \vee \underline{v}_{G\left(e_{i}\right)}\left(x_{j}\right)\right)=1$,
$E_{4}\left(\bar{v}_{F\left(e_{i}\right)}\left(x_{j}\right), \bar{v}_{F\left(e_{i}\right)}\left(x_{j}\right) \vee \bar{v}_{G\left(e_{i}\right)}\left(x_{j}\right)\right)=1$,
$\forall x_{j} \in U, e_{i} \in P$.
$\Leftrightarrow \underline{u}_{F\left(e_{i}\right)}\left(x_{j}\right)=\underline{u}_{F\left(e_{i}\right)}\left(x_{j}\right) \wedge \underline{u}_{G\left(e_{i}\right)}\left(x_{j}\right)$,
$\bar{u}_{F\left(e_{i}\right)}\left(x_{j}\right)=\bar{u}_{F\left(e_{i}\right)}\left(x_{j}\right) \wedge \bar{u}_{G\left(e_{i}\right)}\left(x_{j}\right)$,
$\underline{v}_{F\left(e_{i}\right)}\left(x_{j}\right)=\underline{v}_{F\left(e_{i}\right)}\left(x_{j}\right) \vee \underline{v}_{G\left(e_{i}\right)}\left(x_{j}\right)$,
$\bar{v}_{F\left(e_{i}\right)}\left(x_{j}\right)=\bar{v}_{F\left(e_{i}\right)}\left(x_{j}\right) \vee \bar{v}_{G\left(e_{i}\right)}\left(x_{j}\right)$,
$\forall x_{j} \in U, e_{i} \in P$.
$\Leftrightarrow\left[\underline{u}_{F\left(e_{i}\right)}\left(x_{j}\right), \bar{u}_{F\left(e_{i}\right)}\left(x_{j}\right)\right] \leqslant\left[\underline{u}_{G\left(e_{i}\right)}\left(x_{j}\right), \bar{u}_{G\left(e_{i}\right)}\left(x_{j}\right)\right]$,
$\left[\underline{v}_{F\left(e_{i}\right)}\left(x_{j}\right), \bar{v}_{F\left(e_{i}\right)}\left(x_{j}\right)\right] \geqslant\left[\underline{v}_{G\left(e_{i}\right)}\left(x_{j}\right), \bar{v}_{G\left(e_{i}\right)}\left(x_{j}\right)\right]$,
$\forall x_{j} \in U, e_{i} \in P$.
$\Leftrightarrow(F, P) \subseteq(G, P)$
$\Leftrightarrow(3) I f(F, P) \subseteq(G, P) \subseteq(H, P)$, then for any $x_{j} \in U$,
$e_{i} \in P$,

$\left[\underline{u}_{F\left(e_{i}\right)}\left(x_{j}\right), \bar{u}_{F\left(e_{i}\right)}\left(x_{j}\right)\right] \leqslant\left[\underline{u}_{G\left(e_{i}\right)}\left(x_{j}\right), \bar{u}_{G\left(e_{i}\right)}\left(x_{j}\right)\right] \leqslant$ $\left[\underline{u}_{H\left(e_{i}\right)}\left(x_{j}\right), \bar{u}_{H\left(e_{i}\right)}\left(x_{j}\right)\right]$ and $\left[\underline{v}_{F\left(e_{i}\right)}\left(x_{j}\right), \bar{v}_{F\left(e_{i}\right)}\left(x_{j}\right)\right] \geqslant$ $\left[\underline{v}_{G\left(e_{i}\right)}\left(x_{j}\right), \bar{v}_{G\left(e_{i}\right)}\left(x_{j}\right)\right] \geqslant\left[\underline{v}_{H\left(e_{i}\right)}\left(x_{j}\right), \bar{v}_{H\left(e_{i}\right)}\left(x_{j}\right)\right]$,

so we have

$E_{1}\left(\underline{u}_{H\left(e_{i}\right)}\left(x_{j}\right), \underline{u}_{H\left(e_{i}\right)}\left(x_{j}\right) \wedge \underline{u}_{F\left(e_{i}\right)}\left(x_{j}\right)\right)=E_{1}\left(\underline{u}_{H\left(e_{i}\right)}\left(x_{j}\right)\right.$, $\left.\underline{u}_{F\left(e_{i}\right)}\left(x_{j}\right)\right) \leqslant E_{1}\left(\underline{u}_{G\left(e_{i}\right)}\left(x_{j}\right), \underline{u}_{F\left(e_{i}\right)}\left(x_{j}\right)\right)=E_{1}\left(\underline{u}_{G\left(e_{i}\right)}\left(x_{j}\right)\right.$, $\left.\underline{u}_{G\left(e_{i}\right)}\left(x_{j}\right) \wedge \underline{u}_{F\left(e_{i}\right)}\left(x_{j}\right)\right)$,

$E_{2}\left(\bar{u}_{H\left(e_{i}\right)}\left(x_{j}\right), \bar{u}_{H\left(e_{i}\right)}\left(x_{j}\right) \wedge \bar{u}_{F\left(e_{i}\right)}\left(x_{j}\right)\right)=E_{2}\left(\bar{u}_{H\left(e_{i}\right)}\left(x_{j}\right)\right.$, $\left.\bar{u}_{F\left(e_{i}\right)}\left(x_{j}\right)\right) \leqslant E_{2}\left(\bar{u}_{G\left(e_{i}\right)}\left(x_{j}\right), \bar{u}_{F\left(e_{i}\right)}\left(x_{j}\right)\right)=E_{2}\left(\bar{u}_{G\left(e_{i}\right)}\left(x_{j}\right)\right.$, $\left.\bar{u}_{G\left(e_{i}\right)}\left(x_{j}\right) \wedge \bar{u}_{F\left(e_{i}\right)}\left(x_{j}\right)\right)$,

$E_{3}\left(\underline{v}_{H\left(e_{i}\right)}\left(x_{j}\right), \underline{v}_{H\left(e_{i}\right)}\left(x_{j}\right) \vee \underline{v}_{F\left(e_{i}\right)}\left(x_{j}\right)\right)=E_{3}\left(\underline{v}_{H\left(e_{i}\right)}\left(x_{j}\right)\right.$, $\left.\underline{v}_{F\left(e_{i}\right)}\left(x_{j}\right)\right) \leqslant E_{3}\left(\underline{v}_{G\left(e_{i}\right)}\left(x_{j}\right), \underline{v}_{F\left(e_{i}\right)}\left(x_{j}\right)\right)=E_{3}\left(\underline{v}_{G\left(e_{i}\right)}\left(x_{j}\right)\right.$, $\left.\underline{v}_{G\left(e_{i}\right)}\left(x_{j}\right) \vee \underline{v}_{F\left(e_{i}\right)}\left(x_{j}\right)\right)$,

$E_{4}\left(\bar{v}_{H\left(e_{i}\right)}\left(x_{j}\right), \bar{v}_{H\left(e_{i}\right)}\left(x_{j}\right) \vee \bar{v}_{F\left(e_{i}\right)}\left(x_{j}\right)\right)=E_{4}\left(\bar{v}_{H\left(e_{i}\right)}\left(x_{j}\right)\right.$, $\left.\bar{v}_{F\left(e_{i}\right)}\left(x_{j}\right)\right) \leqslant E_{4}\left(\bar{v}_{G\left(e_{i}\right)}\left(x_{j}\right), \bar{v}_{F\left(e_{i}\right)}\left(x_{j}\right)\right)=E_{4}\left(\bar{v}_{G\left(e_{i}\right)}\left(x_{j}\right)\right.$, $\left.\bar{v}_{G\left(e_{i}\right)}\left(x_{j}\right) \vee \bar{v}_{F\left(e_{i}\right)}\left(x_{j}\right)\right)$,

then we get

$f\left(E_{l}\left(\underline{u}_{H\left(e_{i}\right)}\left(x_{j}\right), \underline{u}_{H\left(e_{i}\right)}\left(x_{j}\right) \wedge \underline{u}_{F\left(e_{i}\right)}\left(x_{j}\right)\right)\right) \geqslant$ $f\left(E_{l}\left(\underline{u}_{G\left(e_{i}\right)}\left(x_{j}\right), \underline{u}_{G\left(e_{i}\right)}\left(x_{j}\right) \wedge \underline{u}_{F\left(e_{i}\right)}\left(x_{j}\right)\right)\right)$, $f\left(E_{2}\left(\bar{u}_{H\left(e_{i}\right)}\left(x_{j}\right), \bar{u}_{H\left(e_{i}\right)}\left(x_{j}\right) \wedge \bar{u}_{F\left(e_{i}\right)}\left(x_{j}\right)\right)\right) \geqslant$ $f\left(E_{2}\left(\bar{u}_{G\left(e_{i}\right)}\left(x_{j}\right), \bar{u}_{G\left(e_{i}\right)}\left(x_{j}\right) \wedge \bar{u}_{F\left(e_{i}\right)}\left(x_{j}\right)\right)\right)$, $f\left(E_{3}\left(\underline{v}_{H\left(e_{i}\right)}\left(x_{j}\right), \underline{v}_{H\left(e_{i}\right)}\left(x_{j}\right) \vee \underline{v}_{F\left(e_{i}\right)}\left(x_{j}\right)\right)\right) \geqslant$ $f\left(E_{3}\left(\underline{v}_{G\left(e_{i}\right)}\left(x_{j}\right), \underline{v}_{G\left(e_{i}\right)}\left(x_{j}\right) \vee \underline{v}_{F\left(e_{i}\right)}\left(x_{j}\right)\right)\right)$, $f\left(E_{4}\left(\bar{v}_{H\left(e_{i}\right)}\left(x_{j}\right), \bar{v}_{H\left(e_{i}\right)}\left(x_{j}\right) \vee \bar{v}_{F\left(e_{i}\right)}\left(x_{j}\right)\right)\right) \geqslant$ $f\left(E_{4}\left(\bar{v}_{G\left(e_{i}\right)}\left(x_{j}\right), \bar{v}_{G\left(e_{i}\right)}\left(x_{j}\right) \vee \bar{v}_{F\left(e_{i}\right)}\left(x_{j}\right)\right)\right)$.

From the property of aggregation operators, we get
$\left[\underline{u}_{\phi_{1}(H, F)\left(e_{i}\right)}\left(x_{j}\right), \bar{u}_{\phi_{1}(H, F)\left(e_{i}\right)}\left(x_{j}\right)\right] \leqslant\left[\underline{u}_{\phi_{1}(G, F)\left(e_{i}\right)}\left(x_{j}\right)\right.$, $\left.\bar{u}_{\phi_{1}(G, F)\left(e_{i}\right)}\left(x_{j}\right)\right] \leqslant\left[\frac{1}{2}, \frac{1}{2}\right]$, $\left[\underline{v}_{\phi_{1}(H, F)\left(e_{i}\right)}\left(x_{j}\right), \bar{v}_{\phi_{1}(H, F)\left(e_{i}\right)}\left(x_{j}\right)\right] \geqslant\left[\underline{v}_{\phi_{1}(G, F)\left(e_{i}\right)}\left(x_{j}\right)\right.$, $\left.\bar{v}_{\phi_{1}(G, F)\left(e_{i}\right)}\left(x_{j}\right)\right] \geqslant\left[\frac{1}{2}, \frac{1}{2}\right]$.

Let $(Q, P) \in \operatorname{IVIFSS}(U)$ and $Q\left(e_{i}\right)=$ $\left.\left\{\left\langle x_{j},[1 / 2,1 / 2]\right),[1 / 2,1 / 2]\right\rangle \mid x_{j} \in U\right\}$ for any $e_{i} \in P$, then we get

$\left(\phi_{1}(H, F), P\right) \subseteq\left(\phi_{1}(G, F), P\right) \subseteq(Q, P)$,

thus,

$D\left(\left(\phi_{1}(H, F), P\right),(Q, P)\right) \geqslant D\left(\left(\phi_{1}(G, F), P\right),(Q, P)\right)$.

By Definition 12 of entropy for IVIFSSs, we get

$I\left(\left(\phi_{1}(H, F), P\right)\right) \leqslant I\left(\left(\phi_{1}(G, F), P\right)\right)$

$\Leftrightarrow J((H, P),(F, P)) \leqslant J((G, P),(F, P))$.

By the similar way, we get

$I\left(\left(\phi_{1}(H, F), P\right)\right) \leqslant I\left(\left(\phi_{1}(H, G), P\right)\right)$

$\Leftrightarrow J((H, P),(F, P)) \leqslant J((H, P),(G, P))$.

Definition 22. Let $(F, P),(G, P) \in \operatorname{IVIFSS}(U)$, assume that: for any $e_{i} \in P$,

$F\left(e_{i}\right)=\left\{\left\langle x_{j}, u_{F\left(e_{i}\right)}\left(x_{j}\right), v_{F\left(e_{i}\right)}\left(x_{j}\right)\right\rangle \mid x_{j} \in U\right\}=\left\{\left\langle x_{j}\right.\right.$, $\left.\left.\left[\underline{u}_{F\left(e_{i}\right)}\left(x_{j}\right), \bar{u}_{F\left(e_{i}\right)}\left(x_{j}\right)\right],\left[\underline{v}_{F\left(e_{i}\right)}\left(x_{j}\right), \bar{v}_{F\left(e_{i}\right)}\left(x_{j}\right)\right]\right\rangle \mid x_{j} \in U\right\}$,

$G\left(e_{i}\right)=\left\{\left\langle x_{j}, u_{G\left(e_{i}\right)}\left(x_{j}\right), v_{G\left(e_{i}\right)}\left(x_{j}\right)\right\rangle \mid x_{j} \in U\right\}=\left\{\left\langle x_{j}\right.\right.$, $\left.\left.\left[\underline{u}_{G\left(e_{i}\right)}\left(x_{j}\right), \bar{u}_{G\left(e_{i}\right)}\left(x_{j}\right)\right],\left[\underline{v}_{G\left(e_{i}\right)}\left(x_{j}\right), \bar{v}_{G\left(e_{i}\right)}\left(x_{j}\right)\right]\right\rangle \mid x_{j} \in U\right\}$.

Suppose that,

(1) $M_{1}$ is a top-aggregation operator,

(2) $M_{1}\left(x_{1}, x_{2}, x_{3}, x_{4}\right) \leqslant M_{2}\left(x_{1}, x_{2}, x_{3}, x_{4}\right)$ for any $x_{1}, x_{2}, x_{3}, x_{4} \in[0,1]$,

(3) $f$ is a strict fuzzy negation,

(4) $E_{l}(l=1,2,3,4)$ are fuzzy equivalence operators,

then for any $\alpha \in[1,+\infty), \beta \in[1,+\infty)$, we can define a new interval-valued intuitionistic fuzzy set $\left(\phi_{2}(F, G), P\right)$ from $(F, P)$ and $(G, P)$ as follows: for any $e_{i} \in P, x_{j} \in U$,

$$
\begin{aligned}
& \underline{u}_{\phi_{2}(F, G)\left(e_{i}\right)}\left(x_{j}\right)=\frac{1}{2}\left\{1-\left[f \left(M _ { 1 } \left(E _ { 1 } \left(\underline{u}_{F\left(e_{i}\right)}\left(x_{j}\right), \underline{u}_{F\left(e_{i}\right)}\left(x_{j}\right)\right.\right.\right.\right.\right. \\
& \left.\wedge \underline{u}_{G\left(e_{i}\right)}\left(x_{j}\right)\right), E_{2}\left(\bar{u}_{F\left(e_{i}\right)}\left(x_{j}\right), \bar{u}_{F\left(e_{i}\right)}\left(x_{j}\right) \wedge \bar{u}_{G\left(e_{i}\right)}\left(x_{j}\right)\right), \\
& E_{3}\left(\underline{v}_{F\left(e_{i}\right)}\left(x_{j}\right), \underline{v}_{F\left(e_{i}\right)}\left(x_{j}\right) \vee \underline{v}_{G\left(e_{i}\right)}\left(x_{j}\right)\right), E_{4}\left(\bar{v}_{F\left(e_{i}\right)}\left(x_{j}\right),\right. \\
& \left.\left.\left.\left.\left.\bar{v}_{F\left(e_{i}\right)}\left(x_{j}\right) \vee \bar{v}_{G\left(e_{i}\right)}\left(x_{j}\right)\right)\right)\right)\right]^{1 / \alpha}\right\} ;
\end{aligned}
$$




$$
\begin{aligned}
& \bar{u}_{\phi_{2}(F, G)\left(e_{i}\right)}\left(x_{j}\right)=\frac{1}{2}\left\{1-\left[f \left(M _ { 1 } \left(E _ { 1 } \left(\underline{u}_{F\left(e_{i}\right)}\left(x_{j}\right), \underline{u}_{F\left(e_{i}\right)}\left(x_{j}\right)\right.\right.\right.\right.\right. \\
& \left.\wedge \underline{u}_{G\left(e_{i}\right)}\left(x_{j}\right)\right), E_{2}\left(\bar{u}_{F\left(e_{i}\right)}\left(x_{j}\right), \bar{u}_{F\left(e_{i}\right)}\left(x_{j}\right) \wedge \bar{u}_{G\left(e_{i}\right)}\left(x_{j}\right)\right), \\
& E_{3}\left(\underline{v}_{F\left(e_{i}\right)}\left(x_{j}\right), \underline{v}_{F\left(e_{i}\right)}\left(x_{j}\right) \vee \underline{v}_{G\left(e_{i}\right)}\left(x_{j}\right)\right), E_{4}\left(\bar{v}_{F\left(e_{i}\right)}\left(x_{j}\right),\right. \\
& \left.\left.\left.\left.\left.\bar{v}_{F\left(e_{i}\right)}\left(x_{j}\right) \vee \bar{v}_{G\left(e_{i}\right)}\left(x_{j}\right)\right)\right)\right)\right]\right\} ; \\
& \underline{v}_{\phi_{2}(F, G)\left(e_{i}\right)}\left(x_{j}\right)=\frac{1}{2}\left\{1+\left[f \left(M _ { 2 } \left(E _ { 1 } \left(\underline{u}_{F\left(e_{i}\right)}\left(x_{j}\right), \underline{u}_{F\left(e_{i}\right)}\left(x_{j}\right)\right.\right.\right.\right.\right. \\
& \left.\wedge \underline{u}_{G\left(e_{i}\right)}\left(x_{j}\right)\right), E_{2}\left(\bar{u}_{F\left(e_{i}\right)}\left(x_{j}\right), \bar{u}_{F\left(e_{i}\right)}\left(x_{j}\right) \wedge \bar{u}_{G\left(e_{i}\right)}\left(x_{j}\right)\right), \\
& E_{3}\left(\underline{v}_{F\left(e_{i}\right)}\left(x_{j}\right), \underline{v}_{F\left(e_{i}\right)}\left(x_{j}\right) \vee \underline{v}_{G\left(e_{i}\right)}\left(x_{j}\right)\right), E_{4}\left(\bar{v}_{F\left(e_{i}\right)}\left(x_{j}\right),\right. \\
& \left.\left.\left.\left.\left.\bar{v}_{F\left(e_{i}\right)}\left(x_{j}\right) \vee \bar{v}_{G\left(e_{i}\right)}\left(x_{j}\right)\right)\right)\right)\right]^{\beta}\right\} ; \\
& \bar{v}_{\phi_{2}(F, G)\left(e_{i}\right)}\left(x_{j}\right)=\frac{1}{2}\left\{1+\left[f \left(M _ { 2 } \left(E _ { 1 } \left(\underline{u}_{F\left(e_{i}\right)}\left(x_{j}\right), \underline{u}_{F\left(e_{i}\right)}\left(x_{j}\right)\right.\right.\right.\right.\right. \\
& \left.\wedge \underline{u}_{G\left(e_{i}\right)}\left(x_{j}\right)\right), E_{2}\left(\bar{u}_{F\left(e_{i}\right)}\left(x_{j}\right), \bar{u}_{F\left(e_{i}\right)}\left(x_{j}\right) \wedge \bar{u}_{G\left(e_{i}\right)}\left(x_{j}\right)\right), \\
& E_{3}\left(\underline{v}_{F\left(e_{i}\right)}\left(x_{j}\right), \underline{v}_{F\left(e_{i}\right)}\left(x_{j}\right) \vee \underline{v}_{G\left(e_{i}\right)}\left(x_{j}\right)\right), E_{4}\left(\bar{v}_{F\left(e_{i}\right)}\left(x_{j}\right),\right. \\
& \left.\left.\left.\left.\left.\bar{v}_{F\left(e_{i}\right)}\left(x_{j}\right) \vee \bar{v}_{G\left(e_{i}\right)}\left(x_{j}\right)\right)\right)\right)\right]\right\} .
\end{aligned}
$$

Theorem 13. Let I be an entropy measure of interval-valued intuitionistic fuzzy soft set. For $(F, P),(G, P) \in I V I F S S(U)$, then $I\left(\left(\phi_{2}(F, G), P\right)\right)$ is an inclusion measure between $(F, P)$ and $(G, P)$.

Definition 23. Let $(F, P),(G, P) \in \operatorname{IVIFSS}(U)$, assume that: for any $e_{i} \in P$,

$F\left(e_{i}\right)=\left\{\left\langle x_{j}, u_{F\left(e_{i}\right)}\left(x_{j}\right), v_{F\left(e_{i}\right)}\left(x_{j}\right)\right\rangle \mid x_{j} \in U\right\}=\left\{\left\langle x_{j}\right.\right.$,

$\left.\left.\left[\underline{u}_{F\left(e_{i}\right)}\left(x_{j}\right), \bar{u}_{F\left(e_{i}\right)}\left(x_{j}\right)\right],\left[\underline{v}_{F\left(e_{i}\right)}\left(x_{j}\right), \bar{v}_{F\left(e_{i}\right)}\left(x_{j}\right)\right]\right\rangle \mid x_{j} \in U\right\}$,

$G\left(e_{i}\right)=\left\{\left\langle x_{j}, u_{G\left(e_{i}\right)}\left(x_{j}\right), v_{G\left(e_{i}\right)}\left(x_{j}\right)\right\rangle \mid x_{j} \in U\right\}=\left\{\left\langle x_{j}\right.\right.$, $\left.\left.\left[\underline{u}_{G\left(e_{i}\right)}\left(x_{j}\right), \bar{u}_{G\left(e_{i}\right)}\left(x_{j}\right)\right],\left[\underline{v}_{G\left(e_{i}\right)}\left(x_{j}\right), \bar{v}_{G\left(e_{i}\right)}\left(x_{j}\right)\right]\right\rangle \mid x_{j} \in U\right\}$.

Suppose that,

(1) $M$ is a top-aggregation operator,

(2) $f$ is a strict fuzzy negation,

(3) $E_{l}(l=1,2,3,4)$ are fuzzy equivalence operators,

then for any $0<\alpha_{1} \leqslant \alpha_{2} \leqslant \alpha_{4} \leqslant \alpha_{3}$, we can define a new interval-valued intuitionistic fuzzy set
$\left(\phi_{3}(F, G), P\right)$ from $(F, P)$ and $(G, P)$ as follows: for any $e_{i} \in P, x_{j} \in U$,

$\underline{u}_{\phi_{3}(F, G)\left(e_{i}\right)}\left(x_{j}\right)=\frac{1}{2}\left\{1-\left[f\left(M\left(E_{1}\left(\underline{u}_{F\left(e_{i}\right)}\left(x_{j}\right), \underline{u}_{F\left(e_{i}\right)}\left(x_{j}\right)\right.\right.\right.\right.\right.$

$\left.\wedge \underline{u}_{G\left(e_{i}\right)}\left(x_{j}\right)\right), E_{2}\left(\bar{u}_{F\left(e_{i}\right)}\left(x_{j}\right), \bar{u}_{F\left(e_{i}\right)}\left(x_{j}\right) \wedge \bar{u}_{G\left(e_{i}\right)}\left(x_{j}\right)\right)$,

$E_{3}\left(\underline{v}_{F\left(e_{i}\right)}\left(x_{j}\right), \underline{v}_{F\left(e_{i}\right)}\left(x_{j}\right) \vee \underline{v}_{G\left(e_{i}\right)}\left(x_{j}\right)\right), E_{4}\left(\bar{v}_{F\left(e_{i}\right)}\left(x_{j}\right)\right.$,

$\left.\left.\left.\left.\left.\bar{v}_{F\left(e_{i}\right)}\left(x_{j}\right) \vee \bar{v}_{G\left(e_{i}\right)}\left(x_{j}\right)\right)\right)\right)\right]^{\alpha_{1}}\right\}$;

$\bar{u}_{\phi_{3}(F, G)\left(e_{i}\right)}\left(x_{j}\right)=\frac{1}{2}\left\{1-\left[f\left(M\left(E_{1}\left(\underline{u}_{F\left(e_{i}\right)}\left(x_{j}\right), \underline{u}_{F\left(e_{i}\right)}\left(x_{j}\right)\right.\right.\right.\right.\right.$

$\left.\wedge \underline{u}_{G\left(e_{i}\right)}\left(x_{j}\right)\right), E_{2}\left(\bar{u}_{F\left(e_{i}\right)}\left(x_{j}\right), \bar{u}_{F\left(e_{i}\right)}\left(x_{j}\right) \wedge \bar{u}_{G\left(e_{i}\right)}\left(x_{j}\right)\right)$,

$E_{3}\left(\underline{v}_{F\left(e_{i}\right)}\left(x_{j}\right), \underline{v}_{F\left(e_{i}\right)}\left(x_{j}\right) \vee \underline{v}_{G\left(e_{i}\right)}\left(x_{j}\right)\right), E_{4}\left(\bar{v}_{F\left(e_{i}\right)}\left(x_{j}\right)\right.$,

$\left.\left.\left.\left.\left.\bar{v}_{F\left(e_{i}\right)}\left(x_{j}\right) \vee \bar{v}_{G\left(e_{i}\right)}\left(x_{j}\right)\right)\right)\right)\right]^{\alpha_{2}}\right\}$;

$\underline{v}_{\phi_{3}(F, G)\left(e_{i}\right)}\left(x_{j}\right)=\frac{1}{2}\left\{1+\left[f\left(M\left(E_{1}\left(\underline{u}_{F\left(e_{i}\right)}\left(x_{j}\right), \underline{u}_{F\left(e_{i}\right)}\left(x_{j}\right)\right.\right.\right.\right.\right.$

$\left.\wedge \underline{u}_{G\left(e_{i}\right)}\left(x_{j}\right)\right), E_{2}\left(\bar{u}_{F\left(e_{i}\right)}\left(x_{j}\right), \bar{u}_{F\left(e_{i}\right)}\left(x_{j}\right) \wedge \bar{u}_{G\left(e_{i}\right)}\left(x_{j}\right)\right)$,

$E_{3}\left(\underline{v}_{F\left(e_{i}\right)}\left(x_{j}\right), \underline{v}_{F\left(e_{i}\right)}\left(x_{j}\right) \vee \underline{v}_{G\left(e_{i}\right)}\left(x_{j}\right)\right), E_{4}\left(\bar{v}_{F\left(e_{i}\right)}\left(x_{j}\right)\right.$,

$\left.\left.\left.\left.\left.\bar{v}_{F\left(e_{i}\right)}\left(x_{j}\right) \vee \bar{v}_{G\left(e_{i}\right)}\left(x_{j}\right)\right)\right)\right)\right]^{\alpha_{3}}\right\}$;

$\bar{v}_{\phi_{3}(F, G)\left(e_{i}\right)}\left(x_{j}\right)=\frac{1}{2}\left\{1+\left[f\left(M\left(E_{1}\left(\underline{u}_{F\left(e_{i}\right)}\left(x_{j}\right), \underline{u}_{F\left(e_{i}\right)}\left(x_{j}\right)\right.\right.\right.\right.\right.$

$\left.\wedge \underline{u}_{G\left(e_{i}\right)}\left(x_{j}\right)\right), E_{2}\left(\bar{u}_{F\left(e_{i}\right)}\left(x_{j}\right), \bar{u}_{F\left(e_{i}\right)}\left(x_{j}\right) \wedge \bar{u}_{G\left(e_{i}\right)}\left(x_{j}\right)\right)$,

$E_{3}\left(\underline{v}_{F\left(e_{i}\right)}\left(x_{j}\right), \underline{v}_{F\left(e_{i}\right)}\left(x_{j}\right) \vee \underline{v}_{G\left(e_{i}\right)}\left(x_{j}\right)\right), E_{4}\left(\bar{v}_{F\left(e_{i}\right)}\left(x_{j}\right)\right.$,

$\left.\left.\left.\left.\left.\bar{v}_{F\left(e_{i}\right)}\left(x_{j}\right) \vee \bar{v}_{G\left(e_{i}\right)}\left(x_{j}\right)\right)\right)\right)\right]^{\alpha_{4}}\right\}$.

Theorem 14. Let I be an entropy measure of interval-valued intuitionistic fuzzy soft set. For $(F, P),(G, P) \in \operatorname{IVIFSS}(U)$, then $I\left(\left(\phi_{3}(F, G), P\right)\right)$ is an inclusion measure between $(F, P)$ and $(G, P)$.

Proof. We only need to prove that all the properties in Definition 11 hold.

(1) If $(F, P)=(U, P),(G, A)=(\emptyset, P)$, we get $F\left(e_{i}\right)=\left\{\left\langle x_{j},[1,1],[0,0]\right\rangle \mid x_{j} \in U\right\}, G\left(e_{i}\right)=$ $\left\{\left\langle x_{j},[0,0],[1,1]\right\rangle \mid x_{j} \in U\right\}$ for $\forall e_{i} \in P$, then we have for any $x_{j} \in U, e_{i} \in P$,

$E_{1}\left(\underline{u}_{F\left(e_{i}\right)}\left(x_{j}\right), \underline{u}_{F\left(e_{i}\right)}\left(x_{j}\right) \wedge \underline{u}_{G\left(e_{i}\right)}\left(x_{j}\right)=E_{1}(1,1 \wedge 0)=\right.$ 0 ,

$E_{2}\left(\bar{u}_{F\left(e_{i}\right)}\left(x_{j}\right), \bar{u}_{F\left(e_{i}\right)}\left(x_{j}\right) \wedge \bar{u}_{G\left(e_{i}\right)}\left(x_{j}\right)\right)=E_{2}(1,1 \wedge$ $0)=0$,

$E_{3}\left(\underline{v}_{F\left(e_{i}\right)}\left(x_{j}\right), \underline{v}_{F\left(e_{i}\right)}\left(x_{j}\right) \vee \underline{v}_{G\left(e_{i}\right)}\left(x_{j}\right)\right)=E_{3}(0,0 \vee 1)=$ 0 ,

$E_{4}\left(\underline{v}_{F\left(e_{i}\right)}\left(x_{j}\right), \underline{v}_{F\left(e_{i}\right)}\left(x_{j}\right) \vee \underline{v}_{G\left(e_{i}\right)}\left(x_{j}\right)\right)=E_{4}(0,0 \vee 1)=$ 
0 ,

so we get,

$M\left(E_{1}\left(\underline{u}_{F\left(e_{i}\right)}\left(x_{j}\right), \underline{u}_{F\left(e_{i}\right)}\left(x_{j}\right) \wedge \underline{u}_{G\left(e_{i}\right)}\left(x_{j}\right)\right), E_{2}\left(\bar{u}_{F\left(e_{i}\right)}\left(x_{j}\right)\right.\right.$,

$\left.\bar{u}_{F\left(e_{i}\right)}\left(x_{j}\right) \wedge \bar{u}_{G\left(e_{i}\right)}\left(x_{j}\right)\right), E_{3}\left(\underline{v}_{F\left(e_{i}\right)}\left(x_{j}\right), \underline{v}_{F\left(e_{i}\right)}\left(x_{j}\right) \vee\right.$

$\left.\left.\underline{v}_{G\left(e_{i}\right)}\left(x_{j}\right)\right), E_{4}\left(\bar{v}_{F\left(e_{i}\right)}\left(x_{j}\right), \bar{v}_{F\left(e_{i}\right)}\left(x_{j}\right) \vee \bar{v}_{G\left(e_{i}\right)}\left(x_{j}\right)\right)\right)$

$=M(0,0,0,0)=0$.

Thus, it is easy to get that

$\left[\underline{u}_{\phi_{3}(F, G)\left(e_{i}\right)}\left(x_{j}\right), \bar{u}_{\phi_{3}(F, G)\left(e_{i}\right)}\left(x_{j}\right)\right]=[0,0]$,

$\left[\underline{v}_{\phi_{3}(F, G)\left(e_{i}\right)}\left(x_{j}\right), \bar{v}_{\phi_{3}(F, G)\left(e_{i}\right)}\left(x_{j}\right)\right]=[1,1]$,

$\forall x_{j} \in U, e_{i} \in P$.

From Definition 12 of entropy for IVIFSSs, we know

$I\left(\left(\phi_{3}(F, G), P\right)\right)=0 \Leftrightarrow J((F, P),(G, P))=0$.

$(2) I\left(\left(\phi_{3}(F, G), P\right)\right)=J((F, P),(G, P))=1$,

$\Leftrightarrow\left[\underline{u}_{\phi_{3}(F, G)\left(e_{i}\right)}\left(x_{j}\right), \bar{u}_{\phi_{3}(F, G)\left(e_{i}\right)}\left(x_{j}\right)\right]=\left[\frac{1}{2}, \frac{1}{2}\right]$,

$\left[\underline{v}_{\phi_{3}(F, G)\left(e_{i}\right)}\left(x_{j}\right), \bar{v}_{\phi_{3}(F, G)\left(e_{i}\right)}\left(x_{j}\right)\right]=\left[\frac{1}{2}, \frac{1}{2}\right]$,

$\forall x_{j} \in U, e_{i} \in P$.

$\Leftrightarrow \quad f\left(M\left(E_{1}\left(\underline{u}_{F\left(e_{i}\right)}\left(x_{j}\right), \underline{u}_{F\left(e_{i}\right)}\left(x_{j}\right) \wedge \underline{u}_{G\left(e_{i}\right)}\left(x_{j}\right)\right)\right.\right.$,

$E_{2}\left(\bar{u}_{F\left(e_{i}\right)}\left(x_{j}\right), \bar{u}_{F\left(e_{i}\right)}\left(x_{j}\right) \wedge \bar{u}_{G\left(e_{i}\right)}\left(x_{j}\right)\right), E_{3}\left(\underline{v}_{F\left(e_{i}\right)}\left(x_{j}\right)\right.$,

$\left.\underline{v}_{F\left(e_{i}\right)}\left(x_{j}\right) \vee \underline{v}_{G\left(e_{i}\right)}\left(x_{j}\right)\right), \quad E_{4}\left(\bar{v}_{F\left(e_{i}\right)}\left(x_{j}\right), \bar{v}_{F\left(e_{i}\right)}\left(x_{j}\right) \vee\right.$

$\left.\left.\left.\bar{v}_{G\left(e_{i}\right)}\left(x_{j}\right)\right)\right)\right)=0, \forall x_{j} \in U, e_{i} \in P$.

$\Leftrightarrow \quad M\left(E_{1}\left(\underline{u}_{F\left(e_{i}\right)}\left(x_{j}\right), \underline{u}_{F\left(e_{i}\right)}\left(x_{j}\right) \wedge \underline{u}_{G\left(e_{i}\right)}\left(x_{j}\right)\right)\right.$,

$E_{2}\left(\bar{u}_{F\left(e_{i}\right)}\left(x_{j}\right), \bar{u}_{F\left(e_{i}\right)}\left(x_{j}\right) \wedge \bar{u}_{G\left(e_{i}\right)}\left(x_{j}\right)\right), E_{3}\left(\underline{v}_{F\left(e_{i}\right)}\left(x_{j}\right)\right.$,

$\left.\underline{v}_{F\left(e_{i}\right)}\left(x_{j}\right) \vee \underline{v}_{G\left(e_{i}\right)}\left(x_{j}\right)\right), \quad E_{4}\left(\bar{v}_{F\left(e_{i}\right)}\left(x_{j}\right), \bar{v}_{F\left(e_{i}\right)}\left(x_{j}\right) \vee\right.$

$\left.\left.\bar{v}_{G\left(e_{i}\right)}\left(x_{j}\right)\right)\right)=1, \forall x_{j} \in U, e_{i} \in P$.

$\Leftrightarrow E_{1}\left(\underline{u}_{F\left(e_{i}\right)}\left(x_{j}\right), \underline{u}_{F\left(e_{i}\right)}\left(x_{j}\right) \wedge \underline{u}_{G\left(e_{i}\right)}\left(x_{j}\right)\right)=E_{2}\left(\bar{u}_{F\left(e_{i}\right)}\left(x_{j}\right)\right.$,

$\left.\bar{u}_{F\left(e_{i}\right)}\left(x_{j}\right) \wedge \bar{u}_{G\left(e_{i}\right)}\left(x_{j}\right)\right)=E_{3}\left(\underline{v}_{F\left(e_{i}\right)}\left(x_{j}\right), \underline{v}_{F\left(e_{i}\right)}\left(x_{j}\right) \vee\right.$

$\left.\underline{v}_{G\left(e_{i}\right)}\left(x_{j}\right)\right)=E_{4}\left(\bar{v}_{F\left(e_{i}\right)}\left(x_{j}\right), \bar{v}_{F\left(e_{i}\right)}\left(x_{j}\right) \vee \bar{v}_{G\left(e_{i}\right)}\left(x_{j}\right)\right)=1$,

$\forall x_{j} \in U, e_{i} \in P$.

$\Leftrightarrow \underline{u}_{F\left(e_{i}\right)}\left(x_{j}\right)=\underline{u}_{F\left(e_{i}\right)}\left(x_{j}\right) \wedge \underline{u}_{G\left(e_{i}\right)}\left(x_{j}\right)$,

$\bar{u}_{F\left(e_{i}\right)}\left(x_{j}\right)=\bar{u}_{F\left(e_{i}\right)}\left(x_{j}\right) \wedge \bar{u}_{G\left(e_{i}\right)}\left(x_{j}\right)$,

$\underline{v}_{F\left(e_{i}\right)}\left(x_{j}\right)=\underline{v}_{F\left(e_{i}\right)}\left(x_{j}\right) \vee \underline{v}_{G\left(e_{i}\right)}\left(x_{j}\right)$,

$\bar{v}_{F\left(e_{i}\right)}\left(x_{j}\right)=\bar{v}_{F\left(e_{i}\right)}\left(x_{j}\right) \vee \bar{v}_{G\left(e_{i}\right)}\left(x_{j}\right)$,

$\forall x_{j} \in U, e_{i} \in P$.

$\Leftrightarrow\left[\underline{u}_{F\left(e_{i}\right)}\left(x_{j}\right), \bar{u}_{F\left(e_{i}\right)}\left(x_{j}\right)\right] \leqslant\left[\underline{u}_{G\left(e_{i}\right)}\left(x_{j}\right), \bar{u}_{G\left(e_{i}\right)}\left(x_{j}\right)\right]$ and $\left[\underline{v}_{F\left(e_{i}\right)}\left(x_{j}\right), \bar{v}_{F\left(e_{i}\right)}\left(x_{j}\right)\right] \geqslant\left[\underline{v}_{G\left(e_{i}\right)}\left(x_{j}\right), \bar{v}_{G\left(e_{i}\right)}\left(x_{j}\right)\right]$,

$\forall x_{j} \in U, e_{i} \in P$.

$\Leftrightarrow(F, P) \subseteq(G, P)$.

(3)If $(F, E) \subseteq(G, P) \subseteq(H, P)$, then

$\left[\underline{u}_{F\left(e_{i}\right)}\left(x_{j}\right), \bar{u}_{F\left(e_{i}\right)}\left(x_{j}\right)\right] \leqslant\left[\underline{u}_{G\left(e_{i}\right)}\left(x_{j}\right), \bar{u}_{G\left(e_{i}\right)}\left(x_{j}\right)\right] \leqslant$ $\left[\underline{u}_{H\left(e_{i}\right)}\left(x_{j}\right), \bar{u}_{H\left(e_{i}\right)}\left(x_{j}\right)\right]$ and

$\left[\underline{v}_{F\left(e_{i}\right)}\left(x_{j}\right), \bar{v}_{F\left(e_{i}\right)}\left(x_{j}\right)\right] \geqslant\left[\underline{v}_{G\left(e_{i}\right)}\left(x_{j}\right), \bar{v}_{G\left(e_{i}\right)}\left(x_{j}\right)\right] \geqslant$

$\left[\underline{v}_{H\left(e_{i}\right)}\left(x_{j}\right), \bar{v}_{H\left(e_{i}\right)}\left(x_{j}\right)\right]$,

$\forall x_{j} \in U, e_{i} \in P$.

So we have for $\forall x_{j} \in U, e_{i} \in P$,

$E_{1}\left(\underline{u}_{H\left(e_{i}\right)}\left(x_{j}\right), \underline{u}_{H\left(e_{i}\right)}\left(x_{j}\right) \wedge \underline{u}_{F\left(e_{i}\right)}\left(x_{j}\right)\right)=E_{1}\left(\underline{u}_{H\left(e_{i}\right)}\left(x_{j}\right)\right.$,
$\left.\underline{u}_{F\left(e_{i}\right)}\left(x_{j}\right)\right) \leqslant E_{1}\left(\underline{u}_{G\left(e_{i}\right)}\left(x_{j}\right), \underline{u}_{F\left(e_{i}\right)}\left(x_{j}\right)\right)=E_{1}\left(\underline{u}_{G\left(e_{i}\right)}\left(x_{j}\right)\right.$, $\left.\underline{u}_{G\left(e_{i}\right)}\left(x_{j}\right) \wedge \underline{u}_{F\left(e_{i}\right)}\left(x_{j}\right)\right)$,

$E_{2}\left(\bar{u}_{H\left(e_{i}\right)}\left(x_{j}\right), \bar{u}_{H\left(e_{i}\right)}\left(x_{j}\right) \wedge \bar{u}_{F\left(e_{i}\right)}\left(x_{j}\right)\right)=E_{2}\left(\bar{u}_{H\left(e_{i}\right)}\left(x_{j}\right)\right.$, $\left.\bar{u}_{F\left(e_{i}\right)}\left(x_{j}\right)\right) \leqslant E_{2}\left(\bar{u}_{G\left(e_{i}\right)}\left(x_{j}\right), \bar{u}_{F\left(e_{i}\right)}\left(x_{j}\right)\right)=E_{2}\left(\bar{u}_{G\left(e_{i}\right)}\left(x_{j}\right)\right.$, $\left.\bar{u}_{G\left(e_{i}\right)}\left(x_{j}\right) \wedge \bar{u}_{F\left(e_{i}\right)}\left(x_{j}\right)\right)$,

$E_{3}\left(\underline{v}_{H\left(e_{i}\right)}\left(x_{j}\right), \underline{v}_{H\left(e_{i}\right)}\left(x_{j}\right) \vee \underline{v}_{F\left(e_{i}\right)}\left(x_{j}\right)\right)=E_{3}\left(\underline{v}_{H\left(e_{i}\right)}\left(x_{j}\right)\right.$, $\left.\underline{v}_{F\left(e_{i}\right)}\left(x_{j}\right)\right) \leqslant E_{3}\left(\underline{v}_{G\left(e_{i}\right)}\left(x_{j}\right), \underline{v}_{F\left(e_{i}\right)}\left(x_{j}\right)\right)=E_{3}\left(\underline{v}_{G\left(e_{i}\right)}\left(x_{j}\right)\right.$, $\left.\underline{v}_{G\left(e_{i}\right)}\left(x_{j}\right) \vee \underline{v}_{F\left(e_{i}\right)}\left(x_{j}\right)\right)$,

$E_{4}\left(\bar{v}_{H\left(e_{i}\right)}\left(x_{j}\right), \bar{v}_{H\left(e_{i}\right)}\left(x_{j}\right) \vee \bar{v}_{F\left(e_{i}\right)}\left(x_{j}\right)\right)=E_{4}\left(\bar{v}_{H\left(e_{i}\right)}\left(x_{j}\right)\right.$, $\left.\bar{v}_{F\left(e_{i}\right)}\left(x_{j}\right)\right) \leqslant E_{4}\left(\bar{v}_{G\left(e_{i}\right)}\left(x_{j}\right), \bar{v}_{F\left(e_{i}\right)}\left(x_{j}\right)\right)=E_{4}\left(\bar{v}_{G\left(e_{i}\right)}\left(x_{j}\right)\right.$, $\left.\bar{v}_{G\left(e_{i}\right)}\left(x_{j}\right) \vee \bar{v}_{F\left(e_{i}\right)}\left(x_{j}\right)\right)$,

so we get

$f\left(M\left(E_{1}\left(\underline{u}_{H\left(e_{i}\right)}\left(x_{j}\right), \underline{u}_{H\left(e_{i}\right)}\left(x_{j}\right) \wedge \underline{u}_{F\left(e_{i}\right)}\left(x_{j}\right)\right), E_{2}\left(\bar{u}_{H\left(e_{i}\right)}\right.\right.\right.$ $\left.\left(x_{j}\right), \bar{u}_{H\left(e_{i}\right)}\left(x_{j}\right) \wedge \bar{u}_{F\left(e_{i}\right)}\left(x_{j}\right)\right), E_{3}\left(\underline{v}_{H\left(e_{i}\right)}\left(x_{j}\right), \underline{v}_{H\left(e_{i}\right)}\left(x_{j}\right)\right.$ $\left.\left.\left.\vee \underline{v}_{F\left(e_{i}\right)}\left(x_{j}\right)\right), E_{4}\left(\bar{v}_{H\left(e_{i}\right)}\left(x_{j}\right), \bar{v}_{H\left(e_{i}\right)}\left(x_{j}\right) \vee \bar{v}_{F\left(e_{i}\right)}\left(x_{j}\right)\right)\right)\right) \geqslant$ $f\left(M\left(E_{1}\left(\underline{u}_{G\left(e_{i}\right)}\left(x_{j}\right), \underline{u}_{G\left(e_{i}\right)}\left(x_{j}\right) \wedge \underline{u}_{F\left(e_{i}\right)}\left(x_{j}\right)\right), E_{2}\left(\bar{u}_{G\left(e_{i}\right)}\right.\right.\right.$ $\left.\left(x_{j}\right), \bar{u}_{G\left(e_{i}\right)}\left(x_{j}\right) \wedge \bar{u}_{F\left(e_{i}\right)}\left(x_{j}\right)\right), E_{3}\left(\underline{v}_{G\left(e_{i}\right)}\left(x_{j}\right), \underline{v}_{G\left(e_{i}\right)}\left(x_{j}\right)\right.$ $\left.\left.\left.\vee \underline{v}_{F\left(e_{i}\right)}\left(x_{j}\right)\right), E_{4}\left(\bar{v}_{G\left(e_{i}\right)}\left(x_{j}\right), \bar{v}_{G\left(e_{i}\right)}\left(x_{j}\right) \vee \bar{v}_{F\left(e_{i}\right)}\left(x_{j}\right)\right)\right)\right)$. Hence,

$\left[\underline{u}_{\phi_{3}(H, F)\left(e_{i}\right)}\left(x_{j}\right), \bar{u}_{\phi_{3}(H, F)\left(e_{i}\right)}\left(x_{j}\right)\right] \leqslant\left[\underline{u}_{\phi_{3}(G, F)\left(e_{i}\right)}\left(x_{j}\right)\right.$, $\left.\bar{u}_{\phi_{3}(G, F)\left(e_{i}\right)}\left(x_{j}\right)\right] \leqslant\left[\frac{1}{2}, \frac{1}{2}\right]$ and $\left[\underline{v}_{\phi_{3}(H, F)\left(e_{i}\right)}\left(x_{j}\right), \bar{v}_{\phi_{3}(H, F)\left(e_{i}\right)}\left(x_{j}\right)\right] \geqslant\left[\underline{v}_{\phi_{3}(G, F)\left(e_{i}\right)}\left(x_{j}\right)\right.$, $\left.\bar{v}_{\phi_{3}(G, F)\left(e_{i}\right)}\left(x_{j}\right)\right] \geqslant\left[\frac{1}{2}, \frac{1}{2}\right], \forall x_{j} \in U, e_{i} \in P$.

Let $(Q, P) \in \operatorname{IVIFSS}(U)$ and $Q\left(e_{i}\right)=$ $\left.\left\{\left\langle x_{j},[1 / 2,1 / 2]\right),[1 / 2,1 / 2]\right\rangle \mid x_{j} \in U\right\}$ for any $e_{i} \in P$, then we get

$\left(\phi_{3}(H, F), P\right) \subseteq\left(\phi_{3}(G, F), P\right) \subseteq(Q, P)$,

by Definition 9 of distance measure for IVIFSSs, we get

$D\left(\left(\phi_{3}(H, F), P\right),(Q, P)\right) \geqslant D\left(\left(\phi_{3}(G, F), P\right),(Q, P)\right)$.

Therefore, by Definition 12 of entropy for IVIF SSs, we get

$I\left(\left(\phi_{3}(H, F), P\right)\right) \leqslant I\left(\left(\phi_{3}(G, F), P\right)\right)$

$\Leftrightarrow J((H, P),(F, P)) \leqslant J((G, P),(F, P))$.

By the similar way, we get

$I\left(\left(\phi_{3}(H, F), P\right)\right) \leqslant I\left(\left(\phi_{3}(H, G), P\right)\right)$

$\Leftrightarrow J((H, P),(F, P)) \leqslant J((H, P),(G, P))$.

Definition 24. Let $(F, P),(G, P) \in \operatorname{IVIFSS}(U)$, assume that: for any $e_{i} \in P$,

$F\left(e_{i}\right)=\left\{\left\langle x_{j}, u_{F\left(e_{i}\right)}\left(x_{j}\right), v_{F\left(e_{i}\right)}\left(x_{j}\right)\right\rangle \mid x_{j} \in U\right\}=\left\{\left\langle x_{j}\right.\right.$,

$\left.\left.\left[\underline{u}_{F\left(e_{i}\right)}\left(x_{j}\right), \bar{u}_{F\left(e_{i}\right)}\left(x_{j}\right)\right],\left[\underline{v}_{F\left(e_{i}\right)}\left(x_{j}\right), \bar{v}_{F\left(e_{i}\right)}\left(x_{j}\right)\right]\right\rangle \mid x_{j} \in U\right\}$,

$G\left(e_{i}\right)=\left\{\left\langle x_{j}, u_{G\left(e_{i}\right)}\left(x_{j}\right), v_{G\left(e_{i}\right)}\left(x_{j}\right)\right\rangle \mid x_{j} \in U\right\}=\left\{\left\langle x_{j}\right.\right.$, $\left.\left.\left[\underline{u}_{G\left(e_{i}\right)}\left(x_{j}\right), \bar{u}_{G\left(e_{i}\right)}\left(x_{j}\right)\right],\left[\underline{v}_{G\left(e_{i}\right)}\left(x_{j}\right), \bar{v}_{G\left(e_{i}\right)}\left(x_{j}\right)\right]\right\rangle \mid x_{j} \in U\right\}$. 
Suppose that,

(1) $M$ is a bottom-aggregation operator,

(2) $f$ is a strict fuzzy negation,

(3) $E_{l}(l=1,2,3,4)$ are fuzzy equivalence operators,

then for any $0<\alpha_{1} \leqslant \alpha_{2} \leqslant \alpha_{4} \leqslant \alpha_{3}$, we can define a new interval-valued intuitionistic fuzzy set $\left(\phi_{4}(F, G), P\right)$ from $(F, P)$ and $(G, P)$ as follows: for any $e_{i} \in P, x_{j} \in U$,

$$
\begin{aligned}
& \underline{u}_{\phi_{4}(F, G)\left(e_{i}\right)}\left(x_{j}\right)=\frac{1}{2}\left\{1-\left[M \left(f \left(E _ { 1 } \left(\underline{u}_{F\left(e_{i}\right)}\left(x_{j}\right), \underline{u}_{F\left(e_{i}\right)}\left(x_{j}\right)\right.\right.\right.\right.\right. \\
& \left.\left.\wedge \underline{u}_{G\left(e_{i}\right)}\left(x_{j}\right)\right)\right), f\left(E_{2}\left(\bar{u}_{F\left(e_{i}\right)}\left(x_{j}\right), \bar{u}_{F\left(e_{i}\right)}\left(x_{j}\right) \wedge \bar{u}_{G\left(e_{i}\right)}\left(x_{j}\right)\right)\right), \\
& f\left(E_{3}\left(\underline{v}_{F\left(e_{i}\right)}\left(x_{j}\right), \underline{v}_{F\left(e_{i}\right)}\left(x_{j}\right) \vee \underline{v}_{G\left(e_{i}\right)}\left(x_{j}\right)\right)\right), f\left(E _ { 4 } \left(\bar{v}_{F\left(e_{i}\right)}\left(x_{j}\right),\right.\right. \\
& \left.\left.\left.\left.\left.\bar{v}_{F\left(e_{i}\right)}\left(x_{j}\right) \vee \bar{v}_{G\left(e_{i}\right)}\left(x_{j}\right)\right)\right)\right]\right]^{\left.\alpha_{1}\right\}}\right\} ; \\
& \bar{u}_{\phi_{4}(F, G)\left(e_{i}\right)}\left(x_{j}\right)=\frac{1}{2}\left\{1-\left[M \left(f \left(E _ { 1 } \left(\underline{u}_{F\left(e_{i}\right)}\left(x_{j}\right), \underline{u}_{F\left(e_{i}\right)}\left(x_{j}\right)\right.\right.\right.\right.\right. \\
& \left.\left.\wedge \underline{u}_{G\left(e_{i}\right)}\left(x_{j}\right)\right)\right), f\left(E_{2}\left(\bar{u}_{F\left(e_{i}\right)}\left(x_{j}\right), \bar{u}_{F\left(e_{i}\right)}\left(x_{j}\right) \wedge \bar{u}_{G\left(e_{i}\right)}\left(x_{j}\right)\right)\right), \\
& f\left(E_{3}\left(\underline{v}_{F\left(e_{i}\right)}\left(x_{j}\right), \underline{v}_{F\left(e_{i}\right)}\left(x_{j}\right) \vee \underline{v}_{G\left(e_{i}\right)}\left(x_{j}\right)\right)\right), f\left(E _ { 4 } \left(\bar{v}_{F\left(e_{i}\right)}\left(x_{j}\right),\right.\right. \\
& \left.\left.\left.\left.\left.\bar{v}_{F\left(e_{i}\right)}\left(x_{j}\right) \vee \bar{v}_{G\left(e_{i}\right)}\left(x_{j}\right)\right)\right)\right)\right]^{\alpha_{2}}\right\} ; \\
& \underline{v}_{\phi_{4}(F, G)\left(e_{i}\right)}\left(x_{j}\right)=\frac{1}{2}\left\{1+\left[M \left(f \left(E _ { 1 } \left(\underline{u}_{F\left(e_{i}\right)}\left(x_{j}\right), \underline{u}_{F\left(e_{i}\right)}\left(x_{j}\right)\right.\right.\right.\right.\right. \\
& \left.\left.\wedge \underline{u}_{G\left(e_{i}\right)}\left(x_{j}\right)\right)\right), f\left(E_{2}\left(\bar{u}_{F\left(e_{i}\right)}\left(x_{j}\right), \bar{u}_{F\left(e_{i}\right)}\left(x_{j}\right) \wedge \bar{u}_{G\left(e_{i}\right)}\left(x_{j}\right)\right)\right), \\
& f\left(E_{3}\left(\underline{v}_{F\left(e_{i}\right)}\left(x_{j}\right), \underline{v}_{F\left(e_{i}\right)}\left(x_{j}\right) \vee \underline{v}_{G\left(e_{i}\right)}\left(x_{j}\right)\right)\right), f\left(E _ { 4 } \left(\bar{v}_{F\left(e_{i}\right)}\left(x_{j}\right),\right.\right. \\
& \left.\left.\left.\left.\left.\bar{v}_{F\left(e_{i}\right)}\left(x_{j}\right) \vee \bar{v}_{G\left(e_{i}\right)}\left(x_{j}\right)\right)\right)\right)\right]^{\alpha_{3}}\right\} ; \\
& \bar{v}_{\phi_{4}(F, G)\left(e_{i}\right)}\left(x_{j}\right)=\frac{1}{2}\left\{1+\left[M \left(f \left(E _ { 1 } \left(\underline{u}_{F\left(e_{i}\right)}\left(x_{j}\right), \underline{u}_{F\left(e_{i}\right)}\left(x_{j}\right)\right.\right.\right.\right.\right. \\
& \left.\left.\wedge \underline{u}_{G\left(e_{i}\right)}\left(x_{j}\right)\right)\right), f\left(E_{2}\left(\bar{u}_{F\left(e_{i}\right)}\left(x_{j}\right), \bar{u}_{F\left(e_{i}\right)}\left(x_{j}\right) \wedge \bar{u}_{G\left(e_{i}\right)}\left(x_{j}\right)\right)\right), \\
& f\left(E_{3}\left(\underline{v}_{F\left(e_{i}\right)}\left(x_{j}\right), \underline{v}_{F\left(e_{i}\right)}\left(x_{j}\right) \vee \underline{v}_{G\left(e_{i}\right)}\left(x_{j}\right)\right)\right), f\left(E _ { 4 } \left(\bar{v}_{F\left(e_{i}\right)}\left(x_{j}\right),\right.\right. \\
& \left.\left.\left.\left.\bar{v}_{F\left(e_{i}\right)}\left(x_{j}\right) \vee \bar{v}_{G\left(e_{i}\right)}\left(x_{j}\right)\right)\right)\right)\right]^{\left.\alpha_{4}\right\} .}
\end{aligned}
$$

Theorem 15. Let I be an entropy measure of interval-valued intuitionistic fuzzy soft set. For $(F, P),(G, P) \in \operatorname{IVIFSS}(U)$, then $I\left(\left(\phi_{4}(F, G), P\right)\right)$ is an inclusion measure between $(F, P)$ and $(G, P)$.

Theorem 16. If I is an entropy measure of IVIFSSs and $\left(\phi_{h}(F, G), P\right)(h=1,2,3,4)$ is given by Definition 21-24, then $I\left(\left(\phi_{h}(F, G)^{C}, P\right)\right)(h=1,2,3,4)$ is also an inclusion measure between $(F, P)$ and $(G, P)$.

Remark 7. Based on Definition 21-24, by selecting different aggregation operators and fuzzy equivalences, we can obtain a large number of IVIF SSs, which can be used to transform an entropy measure into an inclusion measure for IVIF SSs.

Remark 8. In Ref. ${ }^{17}$, the authors provided a specific interval-valued intuitionistic fuzzy set, the entropy of which have been proved the inclusion measure for IVIFSs. If we extend this intervalvalued intuitionistic fuzzy set into IVIFSSs, the corresponding interval-valued intuitionistic fuzzy soft set can be constructed by Definition 24, Theorem 15 and 16 in this work, by selecting a specific aggregation operator, a specific equivalence operator, a specific fuzzy negation operator and several specific power exponents. To a certain degree, our research is the extension of the research in Ref. ${ }^{17}$ based on fuzzy equivalence and aggregation operators. However, even if it degenerates to the IVIFSs situation, all the formulae given by Definition 21-23 in this work are new.

\subsection{Transformation of similarity measures into inclusion measures for IVIF SSS}

Theorem 17. Let $S$ be a similarity measure of interval-valued intuitionistic fuzzy soft sets and $(F, P),(G, P) \in I V I F S S(U)$, then $J((F, P),(G, P))=$ $S((G, P),(F, P) \cup(G, P))$ is an inclusion measure between $(F, P)$ and $(G, P)$.

Proof. We only need to verify that the following three properties of inclusion measure hold.

(1) $J((U, P),(\emptyset, P))=S((\emptyset, P),(U, P))=0$;

(2) $J((F, P),(G, P))=1 \Leftrightarrow S((G, P),(F, P) \cup$ $(G, P))=1 \Leftrightarrow(G, P)=(F, P) \cup(G, P) \Leftrightarrow(F, P) \subseteq$ $(G, P)$.

(3) If $(F, P) \subseteq(G, P) \subseteq(H, P)$, we easily get that $J((H, P),(F, P))=S((F, P),(H, P) \cup(F, P))=$ $S((F, P),(H, P)) \leqslant S((F, P),(G, P))$ $=S((F, P),(G, P) \cup(F, P))=J((G, P),(F, P))$, and

$J((H, P),(F, P))=S((F, P),(H, P) \cup(F, P))=$ $S((F, P),(H, P)) \leqslant S((G, P),(H, P))$ 
$=S((G, P),(H, P) \cup(G, P))=J((H, P),(G, P))$.

So, we have $J((H, P),(F, P)) \leqslant J((G, P),(F, P))$ and $J((H, P),(F, P)) \leqslant J((H, P),(G, P))$.

Thus, $J$ is an inclusion measure of IVIF SSs.

\section{Disease diagnosis based on entropy and distance measure of IVIFSSS}

An application of similarity measure of intuitionistic fuzzy soft set in disease diagnosis can be found in 23. Benefiting from their idea, an application of the entropy and the distance measure of IVIF SSs in disease diagnosis is given. In oder to estimate if an ill person is suffering from a certain disease or not, with the help of experts, we will construct an intervalvalued intuitionistic fuzzy soft set for the disease and an interval-valued intuitionistic fuzzy soft set for the ill person, respectively. The algorithm is stated as follows:

\section{Algorithm 1}

Step 1 . Select the threshold $\alpha \in[0,1]$ for judging the sample set of a disease and the threshold $\beta \in[0,1]$ for assessing if a patient is suffering from a disease or not;

Step 2. Constructs an interval-valued intuitionistic fuzzy soft set $(F, P)$ over $U$ for the disease.

Step 3. Calculate the entropy of $(F, P)$. If $I((F, P))<\alpha,(F, P)$ can be regarded as a sample set for the disease; if else, collect more relevant information and reconstruct the interval-valued intuitionistic fuzzy soft set for the disease;

Step 4. Constructs an interval-valued intuitionistic fuzzy soft set $(G, P)$ over $U$ for the patient;

Step 5. Calculate the distance measure between $(F, P)$ and $(G, P)$, i.e., $D((F, P),(G, P))$;

Step 6. We say the patient is suffering from the disease if $D((F, P),(G, P))<\beta$; if else, we say the patient is not suffering from the disease.

The thresholds $\alpha$ and $\beta$ in Step 1 can be selected according to the actual situation with the help of experts. Step 2 is based on the consideration that if the uncertain degree of an interval-valued intuitionistic fuzzy soft set for the disease is too large, it maybe not suitable to be a reference sample.

Example 10. Assume that our universal set contain three elements $U=\left\{x_{1}, x_{2}, x_{3}\right\}$, where $x_{1}=$ on the first day of illness, $x_{2}=$ on the second day of illness, $x_{3}=$ on the third day of illness. Here the set of parameters $P$ is the set of certain visible symptoms, assume that $P=\left\{e_{1}, e_{2}, e_{3}, e_{4}, e_{5}\right\}$ where $e_{1}=$ fever, $e_{2}=$ cough, $e_{3}=$ vomit, $e_{4}=$ twitch, $e_{5}=$ trouble breathing. We will try to estimate if a patient is suffering from a certain disease or not.

Step 1. Let $\alpha=0.5$ and $\beta=0.1$.

Step 2. Constructs an interval-valued intuitionistic fuzzy soft set $(F, P)$ over $U$ for the disease which can be prepared with the help of experienced doctors:

$F\left(e_{1}\right)=\left\{\left(x_{1},[0.7,0.8],[0.15,0.2]\right),\left(x_{2},[0.6,0.7]\right.\right.$, $\left.[0.15,0.21]),\left(x_{3},[0.55,0.65],[0.15,0.25]\right)\right\}$, $F\left(e_{2}\right)=\left\{\left(x_{1},[0.7,0.8],[0.1,0.2]\right),\left(x_{2},[0.55,0.65]\right.\right.$, $\left.[0.2,0.25]),\left(x_{3},[0.60,0.70],[0.05,0.1]\right)\right\}$, $F\left(e_{3}\right)=\left\{\left(x_{1},[0.7,0.8],[0.1,0.2]\right),\left(x_{2},[0.65,0.75]\right.\right.$, $\left.[0.2,0.25]),\left(x_{3},[0.77,0.88],[0.1,0.1]\right)\right\}$, $F\left(e_{4}\right)=\left\{\left(x_{1},[0.6,0.7],[0.1,0.2]\right),\left(x_{2},[0.55,0.65]\right.\right.$, $\left.[0.2,0.25]),\left(x_{3},[0.66,0.7],[0.05,0.1]\right)\right\}, \quad F\left(e_{5}\right)=$ $\left\{\left(x_{1},[0.6,0.6],[0.2,0.3]\right),\left(x_{2},[0.55,0.60]\right.\right.$, $\left.[0.2,0.25]),\left(x_{3},[0.7,0.8],[0.05,0.1]\right)\right\}$.

Step 3. Calculate the entropy of $(F, P)$. Here we use the entropy measure of IVIF SSs constructed by Theorem 3. Let $D_{2}((F, P),(Q, P))$ be the Normalized hamming distance between $(F, P)$ and $(Q, P)$ and $f^{\prime}(x)=1-x$ for all $x \in[0,1]$. Then we get $I_{2}((F, P))=f^{\prime}\left(2 D_{2}((F, P),(Q, P))\right)=0.49<0.5$, that is to say, $(F, P)$ can be regarded as a sample set for the disease.

Step 4. Constructs an interval-valued intuitionistic fuzzy soft set $(G, P)$ over $U$ based on the data of a patient:

$G\left(e_{1}\right)=\left\{\left(x_{1},[0.7,0.8],[0.15,0.2]\right),\left(x_{2},[0.6,0.7]\right.\right.$, $\left.[0.15,0.21]),\left(x_{3},[0.55,0.75],[0.15,0.25]\right)\right\}$, $G\left(e_{2}\right)=\left\{\left(x_{1},[0.6,0.7],[0.2,0.3]\right),\left(x_{2},[0.55,0.65]\right.\right.$, $\left.[0.2,0.25]),\left(x_{3},[0.7,0.88],[0.05,0.1]\right)\right\}$, $G\left(e_{3}\right)=\left\{\left(x_{1},[0.5,0.6],[0.2,0.3]\right),\left(x_{2},[0.45,0.55]\right.\right.$, $\left.[0.2,0.25]),\left(x_{3},[0.7,0.78],[0.05,0.1]\right)\right\}$, $G\left(e_{4}\right)=\left\{\left(x_{1},[0.3,0.4],[0.3,0.4]\right),\left(x_{2},[0.55,0.65]\right.\right.$, $\left.[0.2,0.25]),\left(x_{3},[0.7,0.88],[0.05,0.1]\right)\right\}$, $G\left(e_{5}\right)=\left\{\left(x_{1},[0.4,0.5],[0.2,0.3]\right),\left(x_{2},[0.35,0.40]\right.\right.$, $\left.[0.2,0.25]),\left(x_{3},[0.7,0.88],[0.05,0.1]\right)\right\}$.

Step 5. Here we use the Normalized hamming distance between $(F, P)$ and $(G, P)$, which is denoted by $D_{2}((F, P),(G, P))$. It is easy to get that 


$$
D_{2}((F, P),(G, P)) \approx 0.067 \text {. }
$$

Step 6. We conclude that the patient is suffering from the disease since

$$
D_{2}((F, P),(G, P))<0.1 \text {. }
$$

\section{Conclusions and Discussion}

In this paper, we give eight general formulae to calculate the distance measures of IVIFSSs by aggregating fuzzy equivalencies. Consistently with a new axiomatic definition of entropy for IVIFSSs, we prove some theorems which demonstrate that distance measures can be transformed into entropies for IVFSSs. Besides, we prove some theorems which demonstrate that entropies can be transformed into the inclusion measure and the similarity measure for IVIF SSs based on fuzzy equivalencies.

\section{Acknowledgments}

This work has been supported by the National Natural Science Foundation of China (Grant Nos. 61473239, 61175044, 61175055, 61603307), the Fundamental Research Funds for the Central Universities of China (Grant Nos. 2682014ZT28, JBK160132) and the Open Research Fund of Key Laboratory of Xihua University(szjj2014-052).

\section{References}

1. L. A. Zadeh, Fuzzy sets, Information and Control 8(1965)338-353.

2. A. DeLuca, S. Termini, A definition of nonprobabilistic entropy in the setting of fuzzy sets theory, Information and Control 20 (1972) 301-312.

3. K.T. Atanassov, Intuitionistic fuzzy sets, Fuzzy Sets Syst. 20 (1986) 87-96.

4. W.L. Gau, D.J. Buehrer, Vague sets, IEEE Trans. Syst. Man Cybernet. 23 (1993) 610-614.

5. K. Atanassov, Operators over interval-valued intuitionistic fuzzy sets, Fuzzy Sets and Systems 64(2)(1994) 159-174.

6. D. Molodtsov, Soft set theory-First results, Comput.Math. Appl. 37(1999)19-31.

7. P.K. Maji, R. Biswas, A.R. Roy, Fuzzy soft sets, $J$. Fuzzy Math. 9(3)(2001) 589-602.
8. P.K. Maji, More on intuitionistic fuzzy soft sets, in Proc. $12^{\text {th }}$ Int. Conf. Rough Sets, Fuzzy Sets, Data Mining and Granular Computing, eds. H. Sakai, M.K. Chakraborty, A.E. Hassanien, D. Slezak, W. Zhu, (Delhi, India, 2009), pp. 231-240.

9. P.K.Maji, R.Biswas, A.R.Roy, Intuitionistic fuzzy soft sets, J. Fuzzy Math. 9(3)(2001)677-692.

10. P.K.Maji, A.R.Roy, R.Biswas, On intuitionistic fuzzy soft sets, J. Fuzzy Math. 12(3)(2004)669-683.

11. X.B. Yang, T.Y. Lin, J.Y. Yang, Y. Li, D. Yu, Combination of interval-valued fuzzy set and soft set, Comput. Math. Appl. 58(3)(2009) 521-527.

12. Y. Jiang, Y. Tang, Q. Chen, H. Liu, J. Tang, Interval-valued intuitionistic fuzzy soft sets and their properties, Comput. Math. Appl. 60(3)(2010) 906918.

13. J.S. Mi, Y. Leung, W.Z. Wu, An uncertainty measure in partition-based fuzzy rough sets, Int. J. General Syst. 34 (2005) 77-90.

14. A.Kolesrov, Limit properties of quasi-arithmetic means, Fuzzy Sets and Systems 124 (2001)65-71.

15. J.C.Fodor,M.Roubens, Fuzzy preference modelling and multicriteria decision support, Springer Netherlands 14 (1994) 149-173.

16. B. Farhadinia, A theoretical development on the entropy of interval-valued fuzzy sets based on the intuitionistic distance and its relationship with similarity measure,Knowledge-Based Systems 39(2013)79-84.

17. Q. Zhang, H. Xing, F. Liu, J. Ye, P. Tang. Some new entropy measures for interval-valued intuitionistic fuzzy sets based on distances and their relationships with similarity and inclusion measures, Inform. Sci. 283 (2014) 55-69.

18. K. Atanassov, G. Gargov, Interval-valued intuitionistic fuzzy sets, Fuzzy Sets and Systems 31(3)(1989) 343-349.

19. P. Burillo, H. Bustince, Entropy on intuitionistic fuzzy sets and on interval-valued fuzzy sets, Fuzzy Sets and Systems 78 (1996) 305-316.

20. I.B. Turksen, Interval-valued fuzzy sets based on normal forms, Fuzzy Sets and Systems 20 (1986) 191210.

21. L.A. Zadeh, Theory of approximate reasoning, in Machine Intelligence, eds. J. Hayes, D. Michie, L.I. Mikulich, (Ellis Horwood, Chichester, 1970), pp. 149-194.

22. E.P. Klement, R. Mesiar, E. Pap, Triangular Norms, Kluwer Academic Publishers, Dordrecht (2000).

23. N. Çağman, I. Deli. Similarity measures of intuitionistic fuzzy soft sets and their decision making. arXiv preprint arXiv:1301.0456, (2013). 